\title{
16. MAGNETIC PROPERTIES OF GABBROS FROM HOLE 735B, SOUTHWEST INDIAN RIDGE1
}

\author{
Eiichi Kikawa ${ }^{2,4}$ and Janet E. Pariso ${ }^{3}$
}

\begin{abstract}
A total of $500.7 \mathrm{~m}$ of continuous, vertical, oceanic gabbroic section was recovered during Leg 118 . The gabbros obtained exhibited various degrees of alteration and deformation, which gave us a good opportunity to study the magnetic properties of oceanic gabbros. Many of these gabbros, which are mainly Fe-Ti oxide gabbros, have strong and unstable secondary magnetic components that were acquired during drilling. Stable inclinations, which are probably in-situ magnetic directions, show a single polarity, with an average value of $66^{\circ}\left( \pm 5^{\circ}\right)$, meaning that the studied 501-m oceanic gabbroic block may be a candidate for the source of the marine magnetic anomaly. This may also imply that the metamorphism of oceanic gabbros causing acquisition of magnetization probably occurred within one geomagnetic polarity chron (about 0.3 to $0.7 \mathrm{~m} . \mathrm{y}$.) after these gabbros formed at the ridge, leading us to conclude that oceanic gabbros record the so-called Vine-Matthews-Morley type of initial magnetization at the ridge. The average intensity value of stable magnetic components of individual samples, which may be a minimum estimate for remanent magnetizations, is $1.6 \mathrm{~A} / \mathrm{m}$. Assuming this magnetic intensity value and a uniform magnetization within an oceanic gabbroic layer having a thickness of $4.5 \mathrm{~km}$ (i.e., whole layer 3), it is possible to explain most of the marine magnetic anomaly. If magnetic properties of the samples obtained from Hole 735B are common to oceanic gabbros, layer 3 may contribute more significantly to seafloor spreading magnetic anomalies than previously thought.
\end{abstract}

\section{INTRODUCTION}

The Vine-Matthews-Morley hypothesis of seafloor spreading magnetic anomalies has been widely applied in marine geological and geophysical studies, and from it the theory of plate tectonics was developed. According to this hypothesis, a record of the rate of oceanic crustal formation and magnetic reversal chronology can be obtained by examining the overlying marine magnetic anomalies. Attempts to identify the source layer for the seafloor spreading magnetic anomaly have been made using inversion of marine magnetic anomaly field data and by directly measuring oceanic basement rocks recovered by dredging, through DSDP/ODP drilling, and by sampling ophiolites. Several indirect tests of the Vine-MatthewsMorley hypothesis have shown that this hypothesis may very likely be correct as a first-order approximation and that the upper extrusive oceanic layer can account for the magnetic anomaly observed at the sea surface (Talwani et al., 1971; Atwater and Mudie, 1973). However, most direct tests have indicated (1) that the magnetic structure of the oceanic crust is very complex, (2) that a contribution from the lower intrusive layers is necessary, and (3) that a modification of the hypothesis is required (Fox and Opdyke, 1973; Kent et al., 1978; Harrison, 1981; Banerjee, 1984; Harrison, 1987).

Because of sampling difficulty, direct measurements of magnetic properties of in-situ lower oceanic crust have been restricted to the sheeted dike complex sampled at DSDP Hole 504B (Smith and Banerjee, 1986; Pariso and Johnson, 1989a) and to dredged and ophiolite samples (Fox and Opdyke, 1973; Kent et al., 1978; Banerjee, 1980; Dunlop and Prevot, 1982). This may indicate that previous studies of the marine magnetic

\footnotetext{
${ }^{1}$ Von Herzen, R. P., Robinson, P. T., et al., 1991. Proc. ODP, Sci. Results, 118: College Station, TX (Ocean Drilling Program).

${ }_{2}$ Marine Geology Department, Geological Survey of Japan, Tsukuba 305, Ibaraki, Japan 3.

${ }^{3}$ School of Oceanography, University of Washington, Seattle, WA 98195 , U.S.A.

${ }^{4}$ Texas A\&M University, Department of Geophysics, College Station, TX 77843-3114, U.S.A.
}

anomaly source layer were not conducted with sufficient information about oceanic crustal magnetization.

Here, we present the results of magnetic studies of gabbroic samples recovered from Hole 735B during Leg 118. This hole is located on the about 12-m.yr.-old magnetic anomaly $5 \mathrm{~A}$ and was penetrated $500.7 \mathrm{~m}$ into the oceanic gabbroic layer. Because of good recovery in this hole (nearly $87 \%$ in total), the present samples represent the first continuous 501 $\mathrm{m}$ of oceanic gabbroic layer at the site.

The magnetic properties reported here are the intensity and inclination of natural remanent magnetization (NRM), the median demagnetizing field (MDF) for NRM, the initial magnetic susceptibility, and the stable inclination. In addition to the data gained from individual properties, relationships between these parameters also provide useful knowledge concerning the magnetic characteristics of plutonic rocks from the oceanic crust.

\section{GEOLOGICAL DESCRIPTION OF SAMPLES}

A total of $435 \mathrm{~m}$ of olivine gabbro, olivine-bearing gabbro, two-pyroxene gabbro, $\mathrm{Fe}-\mathrm{Ti}$ oxide gabbro, troctolite, and microgabbro with rare basalt and trondhjemite was recovered from Hole 735B. Six major lithostratigraphic units were recognized in the sequence, based on igneous mineralogy, mineral compositions, and degree and style of deformation (see "Lithostratigraphy" section, this study, for further detailed descriptions). About 264 samples were collected from the six units and subjected to paleomagnetic measurements. Gabbros from Hole 735B are of various types and exhibit various degrees of metamorphism and alteration, which gives us a good opportunity to study oceanic gabbros. The rock types of individual samples are listed in Table 1.

\section{EXPERIMENTAL PROCEDURES}

Paleomagnetic measurements were performed for 264 minicore samples, both on board the JOIDES Resolution (JR) and in the paleomagnetic laboratory of the Earthquake Research Institute, University of Tokyo (ERI), and the University of Washington (UW). Standard specimens used in this study were in the shape of minicores $2.5 \mathrm{~cm}$ in diameter and 2.1 to $2.7 \mathrm{~cm}$ long. NRM intensities and inclinations were measured 
using a MOLSPIN portable rock magnetometer, a ring core magnetometer (Koyama, 1986) and a Schonstedt spinner magnetometer located aboard the JR and at the ERI and UW, respectively. Stepwise alternating-field demagnetizations (AFD) were performed with a single-axis demagnetizer on board the JR and a two-axes demagnetizer at the ERI and UW. The majority of samples were demagnetized until the magnetization decreased to below $15 \%$ of the NRM. Two samples were not demagnetized on board the JR because their original remanence intensity was almost the noise level of the MOLSPIN magnetometer [Samples 118-735B-23R-4, 26-28 $\mathrm{cm}$ (Pc. 1B) and 118-735B-38R-2, 15-17 cm (Pc. 1B)]. Zijderveld diagrams plotted from demagnetization data were used to determine stable inclinations by performing a least-squares approximation (Zijderveld, 1967).

After the above measurements were taken, the initial magnetic susceptibility of each sample was measured using Bartington magnetic susceptibility meter (Model MSl) and Schonsted direct field susceptibility meter. The latter was used for samples that were too strong for the Bartington meter. Progressive thermal demagnetizations were performed on 10 selected samples using a Schonstedt thermal demagnetizer on board the JR and at the ERI to examine thermomagnetic behaviors of the samples. The specimens were heated and cooled in air between $20^{\circ}$ and $650^{\circ} \mathrm{C}$ at short times (a 20 to 30 -min heating cycle and a 10 - to 20 -min cooling cycle). These short times spent at elevated temperatures were to minimize the effect of oxidation on the samples.

\section{RESULTS}

Results of the magnetic property measurements for individual samples are given in Table 1 . The magnetic properties listed here are NRM intensity $\left(J_{n r m}\right)$, initial susceptibility $(K)$, median demagnetizing field (MDF), NRM inclination $\left(I_{n r m}\right)$, and stable inclination $\left(I_{s}\right)$. The Koenigsberger ratio, $Q(=$ $\left.J_{n r m} / K H\right)$ and the uncorrected stable declination also are listed for convenience. Results of each magnetic property measurement are summarized separately.

\section{NRM Intensity $\left(J_{n r m}\right)$ and Inclination $\left(I_{n r m}\right)$}

The NRM intensities of the present samples vary from 2.60 $\times 10^{-3}$ to $1.31 \times 10^{2} \mathrm{~A} / \mathrm{m}$, a range of about five orders of magnitude (Fig. 1). Because two samples (Samples 118-735B$32 \mathrm{R}-1,64-66 \mathrm{~cm}$ [Pc. 1F] and 118-735B-44R-2, 6-8 cm [Pc. 1A]) saturated the MOLSPIN magnetometer on board the JR, these two samples must have magnetizations greater than 2.5 $\times 10^{3} \mathrm{~A} / \mathrm{m}$; thus, the range of magnetizations observed is probably wider. These very large variations in NRM intensity have never been observed in other oceanic gabbros. In previous studies for oceanic gabbros, both the largest variation and the highest value in NRM intensity were given by Kent et al. (1978). They reported magnetizations varying from 0.01 to $31.4 \mathrm{~A} / \mathrm{m}$, a range about three orders of magnitude. Figure 1 also shows that many of the studied samples have much higher values than the previously reported values of oceanic intrusive rocks. Hayling and Harrison (1986) summarized arithmetic means from 0.48 to $0.89 \mathrm{~A} / \mathrm{m}$ on oceanic gabbros. Overall variation of intensity with depth in recovered samples (Fig. 1) also indicates that values are scattered in each lithologic unit. Many of the intensity values higher than 10 $\mathrm{A} / \mathrm{m}$ are observed in Unit IV, which consists of Fe-Ti oxide gabbro.

NRM inclinations observed here are summarized in Figure 2. As evident from Table 1 and Figure 2, many reversals of NRM inclination were observed in Hole 735B. However, it seems that Unit I is characterized by the positive (re-

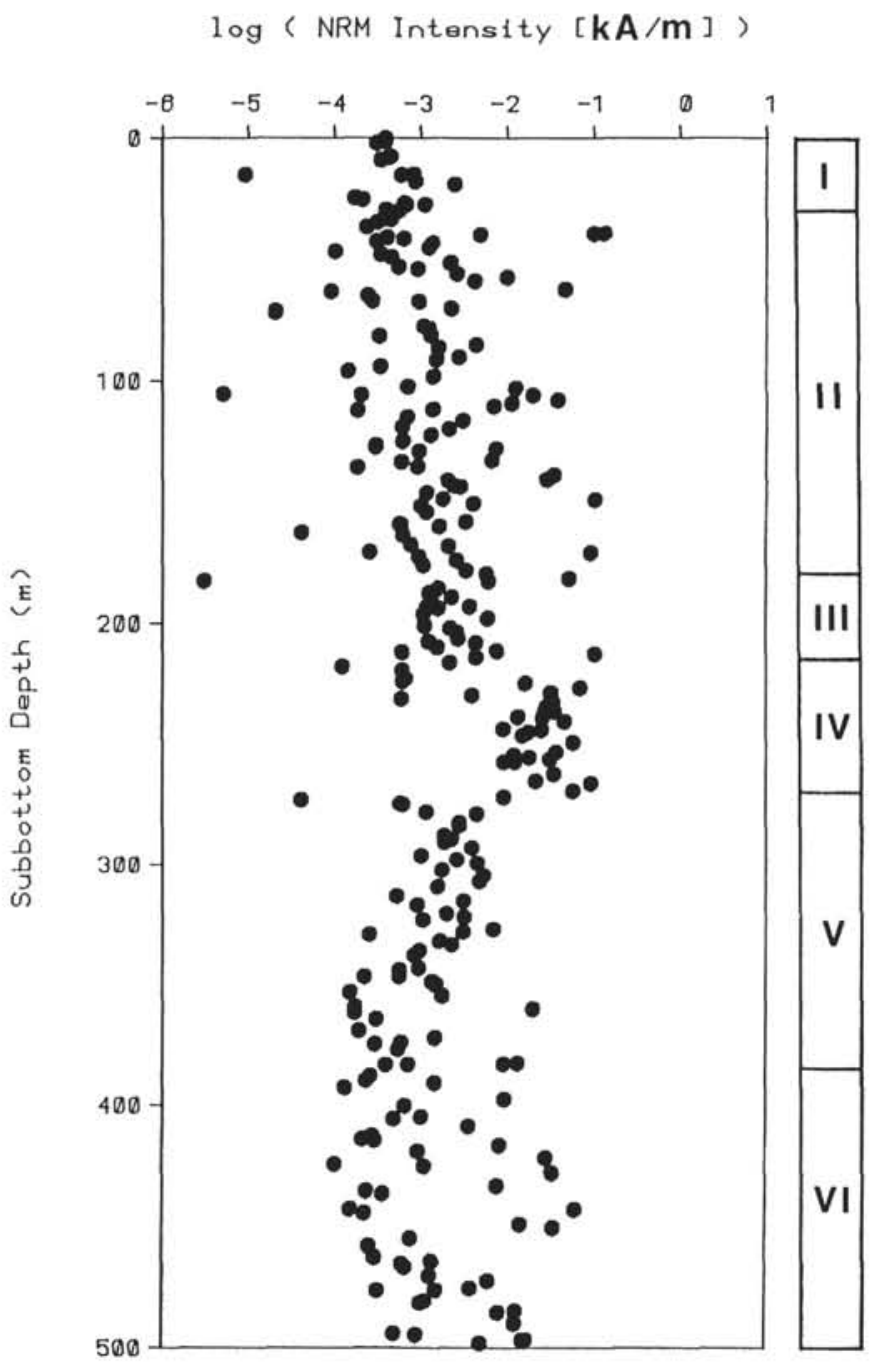

Figure 1. Plot of the intensity of NRM vs. depth in Hole 735B. Lithologic units are shown at the right of figure.

versed) inclinations and that Units IV and VI are mainly composed of negative (normal) inclinations. The mean values for positive and negative inclinations were calculated as $64^{\circ}\left( \pm 9^{\circ}\right)$ and $-62^{\circ}\left( \pm 9^{\circ}\right)$, respectively, using McFadden and Reid's method (1982). Paleomagnetic discussions are given later.

\section{Initial Susceptibility $(\boldsymbol{K})$}

Figure 3 presents a plot of susceptibility vs. sub-bottom depth in Hole 735B. Although the initial susceptibility of the recovered samples has much less scatter than $J_{n r m}$ values, it varies from $3.40 \times 10^{-5}$ to $3.44 \times 10^{-2} \mathrm{cgs}$, a large range of about three orders of magnitude. Some samples were measured by a Schonsted susceptibility meter because they over-ranged the Bartington susceptibility meter $(0.1 \mathrm{cgs})$. Although most of the values measured lie in the range between $10^{-4}$ to $10^{-3} \mathrm{cgs}$, which is consistent with previous studies (Fox and Opdyke, 1973; Kent et al., 1978), many of the samples have much higher values. There seems to be scattered values in each lithological unit. Unit IV is characterized by higher values. The smallest and largest values were observed in foliated metagabbros and in $\mathrm{Fe}$ - $\mathrm{Ti}$ oxide gabbros, respectively. Detailed paleomagnetic features are discussed in a later section. 
Table 1. Magnetic properties of gabbros from Hole 735B.

\begin{tabular}{|c|c|c|c|c|c|c|c|c|c|}
\hline $\begin{array}{l}\text { Core/section } \\
\text { interval }(\mathrm{cm})\end{array}$ & $\begin{array}{l}\text { Depth } \\
\text { (mbsf) }\end{array}$ & $\begin{array}{l}\text { Rock } \\
\text { type }\end{array}$ & $\begin{array}{c}J_{n r m} \\
(\mathrm{kA} / \mathrm{m})\end{array}$ & $\begin{array}{c}K \\
\text { (cgs) }\end{array}$ & $\begin{array}{l}\mathrm{MDF} \\
(\mathrm{mT})\end{array}$ & $Q$ & $I_{n r m}$ & $I_{s}$ & $D_{s}$ \\
\hline \multicolumn{10}{|c|}{$\begin{array}{l}\text { Unit I, foliated metagabbro } \\
118-735 \mathrm{~B} \text { - }\end{array}$} \\
\hline ID $-1,19$ & 0.19 & 1,9 & $3.81 \mathrm{E}-04$ & $5.26 \mathrm{E}-04$ & 24 & 1.9 & 69 & 72 & 333 \\
\hline ID-1, 141 & 1.41 & 1,9 & $3.79 \mathrm{E}-04$ & $2.48 \mathrm{E}-04$ & 23 & 4.0 & 72 & 70 & 135 \\
\hline $1 \mathrm{D}-2,59$ & 2.09 & 1,9 & $2.98 \mathrm{E}-04$ & $1.82 \mathrm{E}-04$ & 36 & 4.3 & 54 & 55 & 307 \\
\hline $2 \mathrm{D}-1,131$ & 7.63 & 1,9 & $4.38 \mathrm{E}-04$ & $3.00 \mathrm{E}-04$ & 7 & 3.8 & 62 & 57 & 137 \\
\hline $2 \mathrm{D}-1,139$ & 7.89 & 1,9 & $4.18 \mathrm{E}-04$ & $4.08 \mathrm{E}-04$ & 18 & 2.7 & 78 & 69 & 320 \\
\hline $2 \mathrm{D}-2,99$ & 8.99 & 1,9 & 3.34E-04 & $2.50 \mathrm{E}-04$ & 14 & 3.5 & 69 & 73 & 315 \\
\hline $3 \mathrm{D}-1,42$ & 14.92 & 1,9 & 8.07E-04 & $4.61 \mathrm{E}-04$ & 28 & 4.6 & 71 & 69 & 164 \\
\hline 3D-1, 58 & 15.08 & 1,9 & $8.74 \mathrm{E}-06$ & $5.90 \mathrm{E}-05$ & $\mathrm{TH}$ & 0.4 & 75 & 74 & 45 \\
\hline $3 \mathrm{D}-1,60$ & 15.10 & 1,9 & $5.86 \mathrm{E}-04$ & $1.99 \mathrm{E}-04$ & - & 7.8 & 76 & 74 & 61 \\
\hline $4 \mathrm{D}-1,32$ & 17.82 & 1,9 & $8.59 \mathrm{E}-04$ & $3.50 \mathrm{E}-04$ & 30 & 6.5 & 71 & 67 & 71 \\
\hline $4 \mathrm{D}-2,7$ & 19.07 & 1,9 & 2.44E-03 & $1.46 \mathrm{E}-03$ & 10 & 4.4 & 57 & 51 & 250 \\
\hline $6 \mathrm{D}-1,56$ & 24.56 & 1,9 & $1.67 \mathrm{E}-04$ & $1.23 \mathrm{E}-04$ & 32 & 3.6 & 80 & 81 & 233 \\
\hline $6 \mathrm{D}-1,111$ & 25.11 & 1,9 & $2.05 \mathrm{E}-04$ & $1.36 \mathrm{E}-04$ & 17 & 4.0 & 68 & 68 & 190 \\
\hline $7 D-1,74$ & 26.74 & 2,9 & 6.37E-04 & 3.74E, 04 & 15 & 4.5 & 75 & 74 & 0 \\
\hline $7 \mathrm{D}-1,134$ & 27.34 & 1,8 & $1.11 \mathrm{E}-03$ & $7.38 \mathrm{E}-04$ & 8 & 4.0 & 77 & 76 & 40 \\
\hline $7 \mathrm{D}-2,9$ & 27.39 & 1,8 & $6.71 \mathrm{E}-04$ & $4.99 \mathrm{E}-04$ & 8 & 3.5 & 86 & 87 & 330 \\
\hline $8 \mathrm{D}-1,22$ & 29.42 & 2,9 & $3.89 \mathrm{E}-04$ & $1.99 \mathrm{E}-04$ & 18 & 5.1 & 63 & 77 & 310 \\
\hline $8 D-1,45$ & 29.65 & 1,9 & $5.62 \mathrm{E}-04$ & 1.12E-04 & 24 & 13.2 & 75 & 75 & 250 \\
\hline 9D-1, 9 & 33.29 & 1,9 & $4.36 \mathrm{E}-04$ & $3.31 \mathrm{E}-04$ & $\mathrm{TH}$ & 3.5 & 61 & 57 & 329 \\
\hline 9D-1, 120 & 34.40 & 2,9 & $3.11 \mathrm{E}-04$ & $3.22 \mathrm{E}-04$ & 13 & 2.5 & 79 & 76 & 159 \\
\hline $10 \mathrm{D}-1,24$ & 36.44 & 2,9 & $2.31 \mathrm{E}-04$ & $2.27 \mathrm{E}-04$ & 30 & 2.7 & 86 & 84 & 231 \\
\hline IID-1, 3 & 39.23 & 1,9 & $1.31 \mathrm{E}-01$ & $5.43 \mathrm{E}-03$ & 5 & 53.6 & -68 & 70 & 317 \\
\hline 11D-1, 6 & 39.26 & 19 & $9.90 \mathrm{E}-02$ & - & - & - & -76 & & \\
\hline
\end{tabular}

Unit II, olivine-bearing and olivine gabbros

\begin{tabular}{|c|c|c|c|c|c|c|c|c|c|}
\hline $12 \mathrm{R}-1,32$ & 39.72 & 1,9 & $4.77 \mathrm{E}-03$ & $3.41 \mathrm{E}-03$ & 9 & 3.7 & 54 & 54 & 53 \\
\hline 12R-1, 131 & 40.71 & 2 & $4.03 \mathrm{E}-04$ & 3.19E-04 & 10 & 3.3 & 70 & 84 & 252 \\
\hline 12R-2, 36 & 41.26 & 2,9 & $6.24 \mathrm{E}-04$ & $1.71 \mathrm{E}-03$ & 16 & 1.0 & 37 & 80 & 208 \\
\hline $12 \mathrm{R}-3,8$ & 42.48 & 5,9 & $2.95 \mathrm{E}-04$ & $9.93 \mathrm{E}-04$ & 6 & 0.8 & 14 & 68 & 61 \\
\hline $12 \mathrm{R}-3,83$ & 43.23 & 1 & $1.33 \mathrm{E}-03$ & $1.94 \mathrm{E}-04$ & 33 & 18.0 & 18 & 18 & 126 \\
\hline $13 \mathrm{R}-1,102$ & 45.02 & 2,9 & $1.22 \mathrm{E}-03$ & $6.40 \mathrm{E}-04$ & 23 & 5.0 & 67 & 67 & 52 \\
\hline $13 \mathrm{R}-2,55$ & 46.55 & 2,9 & $9.69 \mathrm{E}-05$ & 2.78E-04 & 48 & 0.9 & 79 & 75 & 153 \\
\hline $13 R-3,22$ & 47.72 & 2 & $3.42 \mathrm{E}-04$ & 3.33E-04 & 19 & 2.7 & 24 & 69 & 130 \\
\hline $13 \mathrm{R}-3,141$ & 48.91 & 2,9 & $4.50 \mathrm{E}-04$ & $1.04 \mathrm{E}-04$ & 31 & 1.1 & -59 & 57 & 52 \\
\hline $14 \mathrm{R}-1,11$ & 51.31 & 1,9 & $2.19 \mathrm{E}-03$ & $6.68 \mathrm{E}-04$ & 25 & 8.6 & 58 & 59 & 19 \\
\hline $14 \mathrm{R}-1,35$ & 51.55 & 1,9 & $2.15 \mathrm{E}-03$ & $1.90 \mathrm{E}-03$ & 10 & 3.0 & 66 & 69 & 126 \\
\hline $14 \mathrm{R}-2,22$ & 52.92 & 2,9 & $5.44 \mathrm{E}-04$ & $3.77 \mathrm{E}-04$ & 19 & 3.8 & 84 & 78 & 199 \\
\hline $14 \mathrm{R}-3,8$ & 53.77 & 2 & $9.11 \mathrm{E}-04$ & $1.37 \mathrm{E}-03$ & 13 & 1.7 & 56 & 66 & 165 \\
\hline $14 \mathrm{R}-4,22$ & 55.92 & 2,9 & $2.58 \mathrm{E}-03$ & $8.96 \mathrm{E}-04$ & 11 & 7.6 & 69 & 69 & 24 \\
\hline 15R-1, 102 & 57.22 & 2,9 & $9.84 \mathrm{E}-03$ & $3.72 \mathrm{E}-03$ & 3 & 7.0 & -70 & 82 & 356 \\
\hline $15 \mathrm{R}-2,128$ & 58.98 & 1,9 & $4.12 \mathrm{E}-03$ & $1.68 \mathrm{E}-03$ & 3 & 6.5 & -70 & 45 & 261 \\
\hline $16 \mathrm{R}-1,65$ & 62.45 & 1,9 & $4.64 \mathrm{E}-02$ & $1.23 \mathrm{E}-02$ & 4 & 9.9 & -64 & - & - \\
\hline $16 \mathrm{R}-1,139$ & 63.19 & 1,9 & $9.11 \mathrm{E}-05$ & $7.90 \mathrm{E}-05$ & 41 & 3.0 & -19 & 31 & 152 \\
\hline $16 \mathrm{R}-3,9$ & 64.78 & 2,9 & 2.37E-04 & $1.23 \mathrm{E}-04$ & 40 & 5.1 & 75 & 81 & 193 \\
\hline $16 \mathrm{R}-4,77$ & 67.07 & 1 & $2.70 \mathrm{E}-04$ & $5.30 \mathrm{E}-05$ & 42 & 13.4 & 87 & 83 & 194 \\
\hline $16 \mathrm{R}-5,24$ & 67.30 & 6 & $9.37 \mathrm{E}-04$ & $3.24 \mathrm{E}-04$ & 3 & 7.6 & -75 & 63 & 61 \\
\hline $18 \mathrm{R}-2,12$ & 70.12 & 2,9 & $2.20 \mathrm{E}-03$ & $5.35 \mathrm{E}-04$ & 36 & 10.8 & 85 & 83 & 28 \\
\hline $18 \mathrm{R}-2,110$ & 71.10 & 1,9 & $1.90 \mathrm{E}-05$ & $6.70 \mathrm{E}-05$ & 38 & 0.7 & 61 & 67 & 0 \\
\hline $18 \mathrm{R}-3,29$ & 71.79 & 6,9 & $2.33 \mathrm{E}-05$ & $4.30 \mathrm{E}-05$ & 26 & 1.4 & 81 & 79 & 247 \\
\hline $19 \mathrm{R}-2,128$ & 77.48 & 2 & $1.06 \mathrm{E}-03$ & $3.97 \mathrm{E}-04$ & 31 & 7.0 & 43 & 50 & 145 \\
\hline $19 \mathrm{R}-3,73$ & 78.43 & 2 & $1.20 \mathrm{E}-03$ & $5.95 \mathrm{E}-04$ & - & 5.3 & 90 & 81 & 215 \\
\hline $19 \mathrm{R}-5,109$ & 81.37 & 2,9 & $1.27 \mathrm{E}-03$ & $2.30 \mathrm{E}-04$ & 31 & 14.5 & 77 & 66 & 27 \\
\hline $19 \mathrm{R}-5,126$ & 81.54 & 1,9 & $3.23 \mathrm{E}-04$ & $6.73 \mathrm{E}-04$ & 2 & 1.3 & -59 & 79 & 139 \\
\hline $20 \mathrm{R}-1,120$ & 85.40 & 2,9 & $4.26 \mathrm{E}-03$ & $2.74 \mathrm{E}-03$ & 15 & 4.1 & 73 & 78 & 146 \\
\hline 20R-2, 54 & 86.24 & 2 & $1.55 \mathrm{E}-03$ & $5.50 \mathrm{E}-04$ & 35 & 7.4 & 73 & 52 & 48 \\
\hline 21R-1, 91 & 90.11 & 1 & $2.71 \mathrm{E}-03$ & $4.86 \mathrm{E}-04$ & 30 & 14.7 & 61 & 62 & 250 \\
\hline 21R-2, 49 & 91.19 & 2 & $1.48 \mathrm{E}-03$ & $5.41 \mathrm{E}-04$ & 24 & 7.2 & 60 & 71 & 339 \\
\hline $22 \mathrm{R}-1,2$ & 94.02 & 2,9 & $3.25 \mathrm{E}-04$ & $2.83 \mathrm{E}-04$ & 32 & 3.0 & 24 & -3 & 184 \\
\hline $22 \mathrm{R}-2,41$ & 95.91 & 2 & $1.39 \mathrm{E}-04$ & $6.20 \mathrm{E}-04$ & 41 & 0.6 & 69 & 64 & 70 \\
\hline $22 \mathrm{R}-3,118$ & 98.18 & 2,8 & $1.37 \mathrm{E}-03$ & $6.14 \mathrm{E}-04$ & 6 & 5.9 & 80 & 75 & 214 \\
\hline $23 \mathrm{R}-2,34$ & 102.34 & 2 & $6.86 \mathrm{E}-04$ & $3.10 \mathrm{E}-04$ & 19 & 5.8 & 74 & 64 & 175 \\
\hline $23 \mathrm{R}-3,43$ & 102.98 & 3 & $1.22 \mathrm{E}-02$ & $5.46 \mathrm{E}-03$ & 8 & 5.9 & -30 & 52 & 120 \\
\hline $23 \mathrm{R}-4,120$ & 105.20 & 7 & $4.92 \mathrm{E}-06$ & $5.60 \mathrm{E}-05$ & - & 0.2 & 54 & - & - \\
\hline $23 \mathrm{R}-5,13$ & 105.63 & 7 & $2.04 \mathrm{E}-04$ & $1.98 \mathrm{E}-04$ & 27 & 2.7 & 75 & 82 & 111 \\
\hline $24 \mathrm{R}-1,47$ & 105.97 & 2,8 & $1.94 \mathrm{E}-02$ & $1.55 \mathrm{E}-03$ & 5 & 32.9 & 79 & 83 & 255 \\
\hline $24 \mathrm{R}-2,95$ & 107.95 & 3 & $3.76 \mathrm{E}-02$ & $3.44 \mathrm{E}-02$ & 4 & 2.9 & 58 & 51 & 295 \\
\hline $24 \mathrm{R}-3,50$ & 109.00 & 1,9 & $1.09 \mathrm{E}-02$ & - & 23 & - & 76 & 78 & 325 \\
\hline $24 \mathrm{R}-4,26$ & 110.23 & 2 & $6.80 \mathrm{E}-03$ & $3.83 \mathrm{E}-04$ & 4 & 46.7 & 80 & 75 & 112 \\
\hline $25 \mathrm{R}-1,112$ & 111.62 & 1,9 & $1.33 \mathrm{E}-03$ & $2.30 \mathrm{E}-04$ & 24 & 15.2 & 71 & 76 & 55 \\
\hline $25 \mathrm{R}-2,5$ & 112.05 & 1,9 & $1.80 \mathrm{E}-04$ & $6.20 \mathrm{E}-05$ & 26 & 7.7 & 63 & 70 & 19 \\
\hline $25 \mathrm{R}-3,137$ & 114.87 & 2 & $6.78 \mathrm{E}-04$ & $4.81 \mathrm{E}-04$ & 19 & 3.7 & 73 & 73 & 147 \\
\hline $26 \mathrm{R}-1,62$ & 116.22 & 2,8 & $3.00 \mathrm{E}-03$ & $2.04 \mathrm{E}-03$ & 6 & 3.9 & 79 & 73 & 248 \\
\hline $26 \mathrm{R}-3,36$ & 118.86 & 1 & $5.87 \mathrm{E}-04$ & $2.45 \mathrm{E}-04$ & 13 & 6.3 & 71 & 70 & 292 \\
\hline $26 \mathrm{R}-3,112$ & 119.62 & 1 & $2.08 \mathrm{E}-03$ & $4.56 \mathrm{E}-04$ & 21 & 12.0 & 78 & 83 & 102 \\
\hline
\end{tabular}


Table 1 (continued).

\begin{tabular}{|c|c|c|c|c|c|c|c|c|c|}
\hline $\begin{array}{l}\text { Core/section } \\
\text { interval }(\mathrm{cm})\end{array}$ & $\begin{array}{l}\text { Depth } \\
\text { (mbsf) }\end{array}$ & $\begin{array}{l}\text { Rock } \\
\text { type }\end{array}$ & $\underset{(\mathrm{kA} / \mathrm{m})}{J_{n r m}}$ & $\underset{\text { (cgs) }}{K}$ & $\begin{array}{r}\text { MDF } \\
(\mathrm{mT})\end{array}$ & $Q$ & $I_{n r m}$ & $I_{s}$ & $D_{s}$ \\
\hline $27 \mathrm{R}-1,84$ & 122.34 & 2 & $1.27 \mathrm{E}-03$ & $5.64 \mathrm{E}-04$ & 20 & 5.9 & 30 & 31 & 316 \\
\hline $27 \mathrm{R}-3,32$ & 124.82 & 2 & $6.02 \mathrm{E}-04$ & $4.34 \mathrm{E}-04$ & 15 & 3.6 & 75 & 80 & 358 \\
\hline $27 \mathrm{R}-4,52$ & 126.52 & 2 & $2.90 \mathrm{E}-04$ & $3.70 \mathrm{E}-04$ & 20 & 2.1 & 46 & 65 & 61 \\
\hline $28 \mathrm{R}-1,83$ & 127.33 & 2,9 & 2.91E-04 & $1.06 \mathrm{E}-04$ & 27 & 7.2 & 70 & 69 & 279 \\
\hline $28 \mathrm{R}-1,99$ & 127.49 & 2 & $2.45 \mathrm{E}-02$ & - & - & - & - & - & \\
\hline $28 \mathrm{R}-2,12$ & 127.96 & 2,9 & $7.24 \mathrm{E}-03$ & $2.53 \mathrm{E}-03$ & 6 & 7.6 & 68 & 72 & 42 \\
\hline $28 \mathrm{R}-2,114$ & 128.98 & 1 & $9.29 \mathrm{E}-04$ & $2.98 \mathrm{E}-04$ & 22 & 8.2 & 84 & 77 & 72 \\
\hline $28 \mathrm{R}-1,119$ & 132.69 & 2 & $6.54 \mathrm{E}-03$ & $3.43 \mathrm{E}-03$ & 3 & 5.0 & -80 & - & - \\
\hline $29 \mathrm{R}-2,46$ & 133.46 & 2 & $5.76 \mathrm{E}-04$ & $3.84 \mathrm{E}-04$ & 60 & 3.9 & 52 & 83 & 53 \\
\hline $29 \mathrm{R}-3,113$ & 135.44 & 2 & 8.97E-04 & $9.72 \mathrm{E}-04$ & 64 & 2.4 & 39 & 63 & 244 \\
\hline $29 \mathrm{R}-4,19$ & 135.85 & 1,9 & $1.81 \mathrm{E}-04$ & $9.00 \mathrm{E}-05$ & 45 & 5.3 & 60 & 65 & 212 \\
\hline $30 \mathrm{R}-2,97$ & 138.91 & 2,8 & $3.40 \mathrm{E}-02$ & $9.18 \mathrm{E}-03$ & 3 & 9.8 & -44 & 39 & 85 \\
\hline $30 \mathrm{R}-3,137$ & 140.87 & 2,9 & $2.80 \mathrm{E}-02$ & - & 3 & - & -55 & 44 & 316 \\
\hline $30 \mathrm{R}-4,14$ & 141.14 & 2,9 & $1.96 \mathrm{E}-03$ & $1.14 \mathrm{E}-03$ & 2 & 4.5 & -64 & 69 & 44 \\
\hline $30 \mathrm{R}-5,91$ & 143.21 & 1,9 & 2.37E-03 & $2.06 \mathrm{E}-03$ & 5 & 3.0 & -72 & 67 & 181 \\
\hline $31 \mathrm{R}-1,16$ & 143.66 & 2 & $2.79 \mathrm{E}-03$ & $1.31 \mathrm{E}-03$ & 3 & 5.6 & -67 & 73 & 248 \\
\hline $31 \mathrm{R}-2,120$ & 146.20 & 2 & $1.13 \mathrm{E}-03$ & $6.44 \mathrm{E}-04$ & 2 & 4.6 & -75 & 44 & 232 \\
\hline $31 \mathrm{R}-4,118$ & 148.98 & 2,9 & $1.74 \mathrm{E}-03$ & $6.08 \mathrm{E}-04$ & 2 & 7.5 & -76 & 68 & 196 \\
\hline $32 \mathrm{R}-1,64$ & 149.14 & 3 & - & - & - & - & -71 & - & - \\
\hline $32 \mathrm{R}-2,60$ & 150.60 & 2 & $3.94 \mathrm{E}-03$ & $2.80 \mathrm{E}-04$ & 40 & 37.0 & 65 & 68 & 335 \\
\hline $32 \mathrm{R}-3,14$ & 151.64 & 2 & $9.73 \mathrm{E}-04$ & $1.61 \mathrm{E}-04$ & 37 & 15.9 & 66 & 67 & 66 \\
\hline $33 \mathrm{R}-1,81$ & 154.31 & 2 & $1.13 \mathrm{E}-03$ & $1.18 \mathrm{E}-03$ & 39 & 2.5 & -63 & 64 & 1 \\
\hline $33 \mathrm{R}-4,27$ & 158.27 & 2,9 & $3.25 \mathrm{E}-03$ & $5.73 \mathrm{E}-03$ & 2 & 1.5 & -78 & 67 & 20 \\
\hline $33 \mathrm{R}-4,129$ & 159.29 & 6,9 & $5.52 \mathrm{E}-04$ & $6.30 \mathrm{E}-04$ & 3 & 2.3 & -69 & 75 & 135 \\
\hline $34 \mathrm{R}-1,103$ & 159.53 & 2 & $5.65 \mathrm{E}-04$ & $2.40 \mathrm{E}-04$ & 68 & 6.2 & 5 & 78 & 48 \\
\hline $34 \mathrm{R}-2,56$ & 160.36 & 2,9 & $1.59 \mathrm{E}-03$ & $8.51 \mathrm{E}-04$ & TH & 4.9 & -83 & 54 & 3 \\
\hline $34 \mathrm{R}-4,30$ & 162.76 & 1,9 & $4.44 \mathrm{E}-05$ & $3.05 \mathrm{E}-04$ & - & 0.4 & -75 & - & - \\
\hline $35 \mathrm{R}-1,29$ & 163.79 & 2 & $6.01 \mathrm{E}-04$ & $2.24 \mathrm{E}-04$ & 50 & 7.1 & 71 & 69 & 195 \\
\hline $35 \mathrm{R}-3,133$ & 167.65 & 2 & $7.52 \mathrm{E}-04$ & $1.37 \mathrm{E}-04$ & 45 & 14.5 & 44 & 59 & 235 \\
\hline $35 R-4,65$ & 168.47 & 2 & $2.04 \mathrm{E}-03$ & $3.80 \mathrm{E}-04$ & 48 & 1.4 & 40 & 55 & 67 \\
\hline $35 R-5,131$ & 170.81 & 2 & 2.45E-04 & $1.94 \mathrm{E}-04$ & 2 & 3.3 & -71 & 68 & 159 \\
\hline $36 \mathrm{R}-1,14$ & 171.14 & 2,8 & $9.05 \mathrm{E}-02$ & - & 7 & - & -54 & 66 & 51 \\
\hline $36 \mathrm{R}-2,11$ & 172.61 & 2 & $9.22 \mathrm{E}-04$ & $1.10 \mathrm{E}-03$ & 30 & 2.2 & -74 & 79 & 189 \\
\hline $36 \mathrm{R}-3,36$ & 174.36 & 6 & $2.55 \mathrm{E}-03$ & $1.15 \mathrm{E}-03$ & 20 & 5.8 & 75 & 83 & 94 \\
\hline $37 \mathrm{R}-1,11$ & 176.11 & 1 & $1.03 \mathrm{E}-03$ & $1.06 \mathrm{E}-03$ & 32 & 2.6 & -31 & 80 & 140 \\
\hline $37 \mathrm{R}-2,90$ & 178.40 & 2 & $3.23 \mathrm{E}-03$ & $5.92 \mathrm{E}-04$ & 37 & 14.4 & 70 & 75 & 21 \\
\hline $37 \mathrm{R}-3,80$ & 179.80 & 1 & $5.54 \mathrm{E}-03$ & $4.47 \mathrm{E}-03$ & 2 & 3.3 & -67 & 75 & 152 \\
\hline
\end{tabular}

Unit III, olivine and Fe-Ti oxide gabbros

\begin{tabular}{|c|c|c|c|c|c|c|c|c|c|}
\hline $38 \mathrm{R}-1,81$ & 181.81 & 3 & $5.04 \mathrm{E}-02$ & $4.29 \mathrm{E}-03$ & 6 & 30.9 & -62 & - & - \\
\hline $38 \mathrm{R}-2,15$ & 182.65 & 6,9 & $2.60 \mathrm{E}-06$ & $4.80 \mathrm{E}-05$ & - & 0.1 & -61 & - & \\
\hline $38 \mathrm{R}-2,33$ & 182.83 & 6 & $5.87 \mathrm{E}-03$ & $1.87 \mathrm{E}-03$ & 8 & 8.3 & -62 & 71 & 27 \\
\hline $38 \mathrm{R}-4,28$ & 185.78 & 2 & $1.53 \mathrm{E}-03$ & $1.80 \mathrm{E}-03$ & - & 2.2 & 48 & -64 & - \\
\hline $39 \mathrm{R}-1,145$ & 187.45 & 2 & $1.21 \mathrm{E}-03$ & $1.17 \mathrm{E}-03$ & 31 & 2.7 & -49 & 68 & 120 \\
\hline $39 \mathrm{R}-2,68$ & 188.18 & 6 & $1.29 \mathrm{E}-03$ & $1.25 \mathrm{E}-03$ & 58 & 2.7 & 79 & 81 & 261 \\
\hline $39 \mathrm{R}-3,21$ & 189.21 & 2,9 & $2.21 \mathrm{E}-03$ & $3.89 \mathrm{E}-04$ & 38 & 15.0 & 67 & 75 & 130 \\
\hline $40 \mathrm{R}-2,62$ & 193.12 & 1,8 & $1.14 \mathrm{E}-03$ & $2.90 \mathrm{E}-04$ & 21 & 10.4 & 84 & 80 & 130 \\
\hline $40 \mathrm{R}-3,14$ & 193.03 & 1,8 & $3.61 \mathrm{E}-03$ & $1.36 \mathrm{E}-03$ & 3 & 7.0 & -78 & 85 & 315 \\
\hline $40 \mathrm{R}-3,96$ & 193.85 & 1,8 & $1.54 \mathrm{E}-03$ & 4.87E-04 & 43 & 8.3 & 57 & 69 & 29 \\
\hline $40 \mathrm{R}-5,13$ & 196.05 & 1 & $1.04 \mathrm{E}-03$ & $7.49 \mathrm{E}-04$ & 60 & 3.7 & 16 & 68 & 107 \\
\hline 4 IR- 2,30 & 197.72 & 2,8 & $5.85 \mathrm{E}-03$ & $3.10 \mathrm{E}-03$ & 20 & 5.0 & 3 & 67 & 94 \\
\hline $41 \mathrm{R}-4,68$ & 200.86 & 2 & $1.08 \mathrm{E}-03$ & $1.07 \mathrm{E}-03$ & 44 & 2.7 & 65 & 62 & 210 \\
\hline $42 \mathrm{R}-1,94$ & 201.94 & 2 & $2.15 \mathrm{E}-03$ & $4.61 \mathrm{E}-04$ & 62 & 12.3 & 58 & 67 & 20 \\
\hline $42 R-2,119$ & 203.69 & 2 & $2.57 \mathrm{E}-03$ & $1.41 \mathrm{E}-03$ & 2 & 4.8 & -50 & 76 & 24 \\
\hline $42 R-4,62$ & 206.12 & 2 & $2.68 \mathrm{E}-03$ & $1.25 \mathrm{E}-03$ & 24 & 5.7 & -40 & 70 & 353 \\
\hline $43 \mathrm{R}-1,126$ & 207.26 & 1,8 & $1.20 \mathrm{E}-03$ & $1.00 \mathrm{E}-03$ & 37 & 3.2 & 32 & 69 & 125 \\
\hline $43 \mathrm{R}-2,52$ & 207.96 & 2 & $4.25 \mathrm{E}-03$ & $2.12 \mathrm{E}-03$ & 25 & 5.3 & 3 & 65 & 180 \\
\hline $43 R-4,17$ & 209.75 & 2 & $1.53 \mathrm{E}-03$ & $1.08 \mathrm{E}-03$ & 33 & 3.2 & -47 & 71 & 353 \\
\hline $43 R-4,64$ & 211.14 & 2 & $7.42 \mathrm{E}-03$ & $3.15 \mathrm{E}-03$ & 3 & 6.2 & -75 & 62 & 141 \\
\hline $44 \mathrm{R}-1,68$ & 211.68 & 1,8 & $5.85 \mathrm{E}-04$ & $1.87 \mathrm{E}-04$ & 50 & 8.2 & 62 & 68 & 301 \\
\hline $44 \mathrm{R}-2,6$ & 212.56 & 3 & - & $6.62 \mathrm{E}-03$ & - & 39.8 & -81 & - & - \\
\hline $44 \mathrm{R}-2,131$ & 213.81 & 3 & $4.24 \mathrm{E}-03$ & $1.87 \mathrm{E}-03$ & 2 & 6.0 & -78 & 59 & 194 \\
\hline $45 \mathrm{R}-1,1$ & 216.01 & 2 & $2.10 \mathrm{E}-03$ & $1.24 \mathrm{E}-03$ & 4 & 4.5 & -75 & 74 & 219 \\
\hline $45 \mathrm{R}-2,15$ & 217.65 & 2 & $1.18 \mathrm{E}-04$ & $1.05 \mathrm{E}-04$ & 3 & 3.0 & -66 & 31 & 262 \\
\hline $45 \mathrm{R}-3,74$ & 219.26 & 6 & $5.90 \mathrm{E}-04$ & $2.89 \mathrm{E}-04$ & 8 & 5.4 & -67 & 45 & 104 \\
\hline $46 \mathrm{R}-2,21$ & 222.71 & 1,9 & $6.45 \mathrm{E}-04$ & $7.73 \mathrm{E}-04$ & 68 & 2.2 & -2 & 59 & 108 \\
\hline $46 \mathrm{R}-2,128$ & 223.78 & 1,9 & $5.99 \mathrm{E}-04$ & $4.00 \mathrm{E}-04$ & 4 & 3.9 & -71 & 51 & 352 \\
\hline \multicolumn{10}{|c|}{ Unit IV, Fe-Ti oxide gabbro } \\
\hline $46 \mathrm{R}-3,58$ & 224.51 & 2 & $1.58 \mathrm{E}-02$ & $6.38 \mathrm{E}-03$ & 6 & 6.5 & -66 & 72 & 41 \\
\hline $47 \mathrm{R}-1,54$ & 226.54 & 3 & $6.80 \mathrm{E}-02$ & $2.87 \mathrm{E}-02$ & 5 & 6.2 & -83 & 56 & 304 \\
\hline $47 R-2,111$ & 228.56 & 3 & $3.12 \mathrm{E}-02$ & - & 5 & - & -75 & 54 & 102 \\
\hline $47 \mathrm{R}-3,50$ & 229.5 & 2,10 & $3.81 \mathrm{E}-03$ & $1.85 \mathrm{E}-03$ & 4 & 5.4 & -76 & 61 & 288 \\
\hline $47 R-4,64$ & 231.01 & 2 & $5.77 \mathrm{E}-04$ & $1.58 \mathrm{E}-04$ & 53 & 9.6 & 45 & 58 & 43 \\
\hline $48 \mathrm{R}-2,24$ & 232.58 & 3 & $3.32 \mathrm{E}-02$ & $1.34 \mathrm{E}-02$ & 5 & 6.5 & -75 & 77 & 133 \\
\hline
\end{tabular}


Table 1 (continued).

\begin{tabular}{|c|c|c|c|c|c|c|c|c|c|}
\hline $\begin{array}{l}\text { Core/section } \\
\text { interval }(\mathrm{cm})\end{array}$ & $\begin{array}{l}\text { Depth } \\
\text { (mbsf) }\end{array}$ & $\begin{array}{l}\text { Rock } \\
\text { type }\end{array}$ & $\underset{(\mathrm{kA} / \mathrm{m})}{J_{n r m}}$ & $\begin{array}{c}K \\
\text { (cgs) }\end{array}$ & $\begin{array}{r}\text { MDF } \\
(\mathrm{mT})\end{array}$ & $Q$ & $I_{n r m}$ & $I_{s}$ & $D_{s}$ \\
\hline $48 \mathrm{R}-3,53$ & 234.20 & 3 & $2.81 \mathrm{E}-02$ & - & 5 & - & -67 & 63 & 159 \\
\hline $48 \mathrm{R}-4,82$ & 236.32 & 3 & $3.46 \mathrm{E}-02$ & - & 4 & - & -73 & 40 & 117 \\
\hline $49 \mathrm{R}-1,36$ & 236.36 & 3 & $2.68 \mathrm{E}-02$ & $3.89 \mathrm{E}-03$ & $\mathrm{TH}$ & 18.1 & -46 & - & \\
\hline 49R-2, 89 & 238.39 & 3 & $1.31 \mathrm{E}-02$ & - & 3 & - & -61 & 60 & 94 \\
\hline $50 \mathrm{R}-1,77$ & 238.77 & 3 & $2.54 \mathrm{E}-02$ & - & 8 & - & -25 & 57 & 120 \\
\hline $50 \mathrm{R}-2,133$ & 240.42 & 3 & $4.56 \mathrm{E}-02$ & $1.24 \mathrm{E}-02$ & 5 & 9.7 & 67 & - & - \\
\hline $50 \mathrm{R}-4,87$ & 243.37 & 3 & $8.88 \mathrm{E}-03$ & $5.00 \mathrm{E}-03$ & 4 & 4.7 & -58 & 63 & 120 \\
\hline $51 \mathrm{R}-1,102$ & 244.02 & 3 & $2.46 \mathrm{E}-02$ & $1.10 \mathrm{E}-02$ & 3 & 5.9 & -73 & 85 & $i 70$ \\
\hline $51 \mathrm{R}-2,60$ & 244.83 & 3 & $1.75 \mathrm{E}-02$ & $9.52 \mathrm{E}-03$ & 8 & 4.8 & -43 & 53 & 49 \\
\hline $51 \mathrm{R}-3,58$ & 246.08 & 3 & $1.48 \mathrm{E}-02$ & $8.64 \mathrm{E}-03$ & 4 & 4.5 & 71 & 55 & 137 \\
\hline $52 \mathrm{R}-1,115$ & 249.15 & 3 & $5.66 \mathrm{E}-02$ & $5.74 \mathrm{E}-03$ & 2 & 25.9 & -49 & - & - \\
\hline $52 \mathrm{R}-4,69$ & 253.19 & 3 & $3.57 \mathrm{E}-02$ & - & 4 & - & -63 & 42 & 354 \\
\hline $53 \mathrm{R}-1,128$ & 254.28 & 3 & $1.16 \mathrm{E}-02$ & $5.17 \mathrm{E}-03$ & 6 & 5.9 & -55 & 44 & 59 \\
\hline $53 \mathrm{R}-2,94$ & 255.34 & 3 & $1.76 \mathrm{E}-02$ & $6.78 \mathrm{E}-03$ & 6 & 6.8 & -74 & - & - \\
\hline $53 \mathrm{R}-3,15$ & 256.15 & 3 & $3.06 \mathrm{E}-02$ & $5.29 \mathrm{E}-03$ & 4 & 15.2 & -60 & 17 & 3 \\
\hline $53 \mathrm{R}-3,95$ & 256.95 & 3 & $1.20 \mathrm{E}-02$ & - & 5 & - & -85 & 58 & 59 \\
\hline $53 R-4,18$ & 256.98 & 3 & $8.95 \mathrm{E}-03$ & $5.64 \mathrm{E}-03$ & 4 & 4.2 & -77 & 69 & 120 \\
\hline $54 \mathrm{R}-3,125$ & 262.25 & 2 & $3.40 \mathrm{E}-02$ & - & 4 & - & -75 & 54 & 138 \\
\hline $54 \mathrm{R}-5,117$ & 265.17 & 3 & $2.10 \mathrm{E}-02$ & - & - & - & -58 & - & \\
\hline $55 \mathrm{R}-1,107$ & 266.07 & 3 & $9.08 \mathrm{E}-02$ & $8.79 \mathrm{E}-03$ & TH & 27.2 & -57 & - & - \\
\hline $55 \mathrm{R}-3,130$ & 269.30 & 3,9 & $5.67 \mathrm{E}-02$ & - & 4 & - & -78 & 39 & 171 \\
\hline $56 \mathrm{R}-2,11$ & 271.61 & 3,9 & $8.95 \mathrm{E}-03$ & $4.21 \mathrm{E}-03$ & 4 & 5.6 & -67 & 52 & 315 \\
\hline $56 \mathrm{R}-2,144$ & 272.94 & 1,9 & $4.14 \mathrm{E}-05$ & $1.77 \mathrm{E}-04$ & 60 & 0.6 & 2 & 47 & 243 \\
\hline \multicolumn{10}{|c|}{ Unit $\mathrm{V}$, olivine gabbro } \\
\hline $56 \mathrm{R}-4,11$ & 274.38 & 1,8 & $5.73 \mathrm{E}-04$ & $3.07 \mathrm{E}-04$ & 24 & 4.9 & 17 & 70 & 85 \\
\hline $56 \mathrm{R}-4,58$ & 274.81 & 1,8 & $6.11 \mathrm{E}-04$ & $1.69 \mathrm{E}-04$ & 41 & 9.5 & 61 & 70 & 218 \\
\hline 57R-2, 135 & 277.85 & 2 & $1.15 \mathrm{E}-03$ & $1.09 \mathrm{E}-03$ & 70 & 2.8 & 5 & 76 & 224 \\
\hline $57 \mathrm{R}-3,71$ & 278.64 & 2 & $4.41 \mathrm{E}-03$ & $1.47 \mathrm{E}-03$ & 38 & 7.9 & 17 & 52 & 159 \\
\hline $58 \mathrm{R}-2,33$ & 282.33 & 2 & $2.75 \mathrm{E}-03$ & $8.52 \mathrm{E}-04$ & 34 & 8.5 & 59 & -68 & 355 \\
\hline $58 \mathrm{R}-3,34$ & 283.59 & 2 & $2.73 \mathrm{E}-03$ & $6.05 \mathrm{E}-04$ & 27 & 11.9 & 61 & 75 & 109 \\
\hline $59 \mathrm{R}-2,96$ & 287.74 & 2 & $1.83 \mathrm{E}-03$ & $7.41 \mathrm{E}-04$ & 50 & 6.5 & 24 & 71 & 104 \\
\hline $59 \mathrm{R}-3,70$ & 289.20 & 2 & $2.23 \mathrm{E}-03$ & $4.75 \mathrm{E}-04$ & 90 & 12.4 & 63 & 76 & 28 \\
\hline $60 \mathrm{R}-1,18$ & 290.68 & 2 & $1.86 \mathrm{E}-03$ & $3.89 \mathrm{E}-04$ & 90 & 12.6 & 81 & 74 & 218 \\
\hline $60 \mathrm{R}-2,120$ & 292.84 & 2 & $3.83 \mathrm{E}-03$ & $1.32 \mathrm{E}-03$ & 50 & 7.6 & 16 & 67 & 121 \\
\hline $6 \mathrm{IR}-1,81$ & 296.31 & 2 & $9.93 \mathrm{E}-04$ & $6.45 \mathrm{E}-04$ & 55 & 4.0 & 26 & 52 & 67 \\
\hline $61 \mathrm{R}-2,88$ & 297.86 & 2 & $2.59 \mathrm{E}-03$ & $1.51 \mathrm{E}-03$ & 50 & 4.5 & -16 & 72 & 339 \\
\hline $61 \mathrm{R}-3,90$ & 299.33 & 2 & $4.48 \mathrm{E}-03$ & $1.41 \mathrm{E}-03$ & 55 & 8.4 & 65 & 73 & 194 \\
\hline $62 \mathrm{R}-2,45$ & 302.19 & 2 & $1.74 \mathrm{E}-03$ & $9.84 \mathrm{E}-04$ & 58 & 4.7 & 10 & 75 & 76 \\
\hline $62 \mathrm{R}-3,104$ & 304.54 & 2 & $5.27 \mathrm{E}-03$ & $8.71 \mathrm{E}-04$ & 41 & 15.9 & 72 & 80 & 324 \\
\hline $63 \mathrm{R}-2,23$ & 306.96 & 2 & $4.73 \mathrm{E}-03$ & $5.14 \mathrm{E}-04$ & 23 & 24.2 & 79 & 76 & 298 \\
\hline $63 \mathrm{R}-3,80$ & 309.30 & 2 & $1.55 \mathrm{E}-03$ & $5.10 \mathrm{E}-04$ & 36 & 8.0 & 71 & 68 & 133 \\
\hline $63 R-6,28$ & 313.28 & 2,8 & $5.17 \mathrm{E}-04$ & $7.94 \mathrm{E}-04$ & 58 & 1.7 & -52 & 66 & 321 \\
\hline $64 \mathrm{R}-1,33$ & 315.33 & 2,8 & $3.11 \mathrm{E}-03$ & $5.77 \mathrm{E}-04$ & 38 & 14.2 & 79 & 77 & 313 \\
\hline $64 \mathrm{R}-2,54$ & 317.04 & 2 & $9.10 \mathrm{E}-04$ & $1.09 \mathrm{E}-03$ & 38 & 2.2 & -38 & 76 & 230 \\
\hline $65 \mathrm{R}-1,70$ & 320.70 & 2 & $1.98 \mathrm{E}-03$ & $4.25 \mathrm{E}-04$ & 39 & 12.3 & 71 & 79 & 312 \\
\hline $65 \mathrm{R}-2,67$ & 322.17 & 2 & $3.14 \mathrm{E}-03$ & $4.87 \mathrm{E}-04$ & 31 & 17.0 & 63 & 72 & 12 \\
\hline $65 \mathrm{R}-3,61$ & 323.21 & 2 & $1.04 \mathrm{E}-03$ & $6.74 \mathrm{E}-04$ & 70 & 4.1 & 26 & 87 & 37 \\
\hline $66 \mathrm{R}-2,86$ & 327.36 & 1,8 & $6.78 \mathrm{E}-03$ & $3.64 \mathrm{E}-04$ & 26 & 49.0 & 57 & 52 & 331 \\
\hline $66 \mathrm{R}-3,60$ & 328.35 & 1,8 & $3.04 \mathrm{E}-03$ & $1.37 \mathrm{E}-04$ & 32 & 58.4 & 38 & 39 & 356 \\
\hline $66 \mathrm{R}-3,134$ & 329.34 & 1,9 & $2.53 \mathrm{E}-04$ & $3.40 \mathrm{E}-05$ & - & 19.6 & -63 & - & - \\
\hline $67 R-2,86$ & 332.26 & 2 & $1.63 \mathrm{E}-03$ & $7.13 \mathrm{E}-04$ & 53 & 6.0 & 62 & 71 & 106 \\
\hline $67 \mathrm{R}-3,90$ & 333.72 & 2 & $2.26 \mathrm{E}-03$ & $1.54 \mathrm{E}-04$ & 32 & 38.6 & 64 & 67 & 42 \\
\hline $68 \mathrm{R}-1,119$ & 336.19 & 2,8 & $9.52 \mathrm{E}-04$ & $8.36 \mathrm{E}-04$ & 72 & 3.0 & 16 & 66 & 193 \\
\hline $68 \mathrm{R}-3,15$ & 338.15 & 2,8 & 8.31 E- 04 & $7.16 \mathrm{E}-04$ & 2 & 3.1 & -56 & 71 & 38 \\
\hline $69 \mathrm{R}-2,120$ & 343.48 & 2,8 & $9.44 \mathrm{E}-04$ & $3.89 \mathrm{E}-04$ & 56 & 6.4 & 68 & 76 & 198 \\
\hline $69 \mathrm{R}-3,71$ & 344.31 & 2 & $5.62 \mathrm{E}-04$ & $3.80 \mathrm{E}-04$ & 54 & 3.9 & 57 & 81 & 243 \\
\hline $69 \mathrm{R}-4,138$ & 346.88 & 2 & $5.56 \mathrm{E}-04$ & $3.04 \mathrm{E}-04$ & 46 & 4.8 & 22 & 68 & 30 \\
\hline $70 \mathrm{R}-1,105$ & 347.05 & 2,8 & $2.15 \mathrm{E}-04$ & $6.70 \mathrm{E}-05$ & 28 & 8.5 & 54 & 62 & 312 \\
\hline $70 \mathrm{R}-3,4$ & 349.04 & 2 & $1.34 \mathrm{E}-03$ & $6.31 \mathrm{E}-04$ & 45 & 5.6 & 17 & 47 & 60 \\
\hline $70 R-4,33$ & 350.39 & 2,8 & $1.50 \mathrm{E}-03$ & $4.24 \mathrm{E}-04$ & 30 & 9.3 & 81 & 82 & 249 \\
\hline $72 \mathrm{R}-2,82$ & 353.32 & 2 & $1.48 \mathrm{E}-04$ & $4.40 \mathrm{E}-04$ & 80 & 0.9 & -28 & 75 & 133 \\
\hline $71 \mathrm{R}-3,98$ & 354.83 & 2 & $1.75 \mathrm{E}-03$ & $7.34 \mathrm{E}-04$ & 25 & 6.3 & -2 & 55 & 68 \\
\hline $72 \mathrm{R}-3,36$ & 359.36 & 2 & $1.74 \mathrm{E}-04$ & 7.70 E-05 & 90 & 5.9 & 78 & 72 & 217 \\
\hline $72 R-4,39$ & 360.39 & 2,8 & $1.96 \mathrm{E}-02$ & $9.13 \mathrm{E}-03$ & 4 & 5.6 & -74 & 67 & 94 \\
\hline $72 R-5,34$ & 361.69 & 2,8 & $1.71 \mathrm{E}-04$ & $3.00 \mathrm{E}-04$ & 75 & 1.5 & -38 & 66 & 135 \\
\hline $72 R-6,106$ & 364.56 & 2 & $2.98 \mathrm{E}-04$ & $2.65 \mathrm{E}-04$ & 65 & 3.0 & -44 & 68 & 48 \\
\hline $73 \mathrm{R}-3,73$ & 369.23 & 2 & $1.91 \mathrm{E}-04$ & $7.00 \mathrm{E}-05$ & 46 & 7.2 & 67 & 67 & 235 \\
\hline $73 R-5,106$ & 372.09 & 2,8 & $1.45 \mathrm{E}-03$ & $5.14 \mathrm{E}-04$ & 4 & 7.4 & -62 & 62 & 18 \\
\hline $73 \mathrm{R}-7,31$ & 374.08 & 2,8 & $5.80 \mathrm{E}-04$ & $3.26 \mathrm{E}-04$ & 55 & 4.7 & 31 & 70 & 62 \\
\hline $73 \mathrm{R}-7,72$ & 374.79 & 2,8 & $2.87 \mathrm{E}-04$ & $2.61 \mathrm{E}-04$ & 65 & 2.9 & 79 & 80 & 117 \\
\hline $74 \mathrm{R}-2,38$ & 376.88 & 2 & $5.37 \mathrm{E}-04$ & $1.50 \mathrm{E}-04$ & 80 & 9.4 & 47 & 58 & 191 \\
\hline $74 \mathrm{R}-5,37$ & 383.26 & 2,10 & $7.08 \mathrm{E}-04$ & $5.31 \mathrm{E}-04$ & 58 & 3.5 & 30 & 69 & 183 \\
\hline $74 R-6,16$ & 382.47 & 6 & $1.03 \mathrm{E}-02$ & $8.44 \mathrm{E}-03$ & 7 & 3.2 & -76 & 85 & 243 \\
\hline $74 \mathrm{R}-6,41$ & 382.91 & 6 & $9.01 \mathrm{E}-03$ & $5.61 \mathrm{E}-03$ & 5 & 4.2 & -71 & 42 & 15 \\
\hline $74 \mathrm{R}-6,101$ & 383.32 & 2 & $3.91 \mathrm{E}-04$ & $3.67 \mathrm{E}-04$ & 51 & 2.8 & -6 & 62 & 13 \\
\hline
\end{tabular}


Table 1 (continued),

\begin{tabular}{lcllcrrrrr}
\hline $\begin{array}{c}\text { Core/section } \\
\text { interval }(\mathrm{cm})\end{array}$ & $\begin{array}{c}\text { Depth } \\
(\mathrm{mbsf})\end{array}$ & $\begin{array}{c}\text { Rock } \\
\text { type }\end{array}$ & $\begin{array}{c}J_{\text {nrm }} \\
(\mathrm{kA} / \mathrm{m})\end{array}$ & $\begin{array}{c}K \\
(\mathrm{cgs})\end{array}$ & $\begin{array}{r}\text { MDF } \\
(\mathrm{mT})\end{array}$ & $Q$ & $I_{n r m}$ & $I_{s}$ & $D_{s}$ \\
\hline $75 \mathrm{R}-3,48$ & 387.98 & 2 & $2.60 \mathrm{E}-04$ & $2.46 \mathrm{E}-04$ & 27 & 2.8 & -16 & 79 & 323 \\
$75 \mathrm{R}-4,117$ & 389.83 & 2 & $2.34 \mathrm{E}-04$ & $1.37 \mathrm{E}-04$ & 63 & 4.5 & 74 & - & - \\
$75 \mathrm{R}-5,84$ & 390.90 & 2 & $1.44 \mathrm{E}-03$ & $2.89 \mathrm{E}-04$ & 50 & 13.1 & 73 & 74 & 261 \\
$75 \mathrm{R}-6,75$ & 392.75 & 2 & $1.26 \mathrm{E}-04$ & $1.20 \mathrm{E}-04$ & 3 & 2.8 & -79 & 57 & 185 \\
$76 \mathrm{R}-3,50$ & 397.50 & 4 & $9.14 \mathrm{E}-03$ & $3.65 \mathrm{E}-03$ & 3 & 6.6 & -72 & 65 & 170 \\
$76 \mathrm{R}-5,91$ & 400.33 & 2,8 & $6.36 \mathrm{E}-04$ & $5.01 \mathrm{E}-04$ & 28 & 3.3 & -45 & 70 & 149
\end{tabular}

Unit VI, olivine-rich gabbro and troctolite

\begin{tabular}{|c|c|c|c|c|c|c|c|c|c|}
\hline $77 \mathrm{R}-1,135$ & 404.85 & 2,9 & $9.96 \mathrm{E}-04$ & $7.22 \mathrm{E}-04$ & 29 & 3.6 & -42 & 69 & 32 \\
\hline $77 \mathrm{R}-2,63$ & 405.63 & 2,9 & $4.82 \mathrm{E}-04$ & $7.93 \mathrm{E}-04$ & 86 & 1.6 & -35 & 72 & 13 \\
\hline $77 \mathrm{R}-4,70$ & 408.70 & 1,9 & $3.54 \mathrm{E}-03$ & $2.09 \mathrm{E}-03$ & 3 & 4.5 & -66 & 51 & 82 \\
\hline $78 \mathrm{R}-3,51$ & 412.79 & 2 & $2.74 \mathrm{E}-04$ & $2.05 \mathrm{E}-04$ & 3 & 3.5 & -76 & 78 & 307 \\
\hline $78 R-4,34$ & 414.12 & 2,9 & $2.05 \mathrm{E}-04$ & $2.40 \mathrm{E}-04$ & 56 & 2.3 & -24 & 72 & 175 \\
\hline $78 R-4,65$ & 414.65 & 2,9 & 2.93E-04 & $1.26 \mathrm{E}-04$ & 3 & 6.1 & -71 & 59 & 120 \\
\hline $79 R-2,65$ & 416.65 & 1,9 & $8.04 \mathrm{E}-03$ & $4.52 \mathrm{E}-03$ & 3 & 4.7 & -85 & 43 & 146 \\
\hline $79 R-4,12$ & 419.07 & 5 & $9.18 \mathrm{E}-04$ & $2.84 \mathrm{E}-04$ & 8 & 8.5 & -41 & 45 & 65 \\
\hline $79 R-6,27$ & 422.05 & 1,9 & 2.79E-02 & $1.36 \mathrm{E}-02$ & 4 & 5.4 & -60 & 68 & 44 \\
\hline $79 \mathrm{R}-7,99$ & 424.49 & 5 & $1.03 \mathrm{E}-04$ & $1.31 \mathrm{E}-04$ & 3 & 2.1 & -60 & 54 & 231 \\
\hline $80 \mathrm{R}-1,131$ & 425.31 & 2,9 & $1.09 \mathrm{E}-03$ & $9.65 \mathrm{E}-04$ & 4 & 3.0 & -75 & 57 & 211 \\
\hline $80 \mathrm{R}-3,124$ & 428.18 & 2,9 & $3.27 \mathrm{E}-02$ & $5.98 \mathrm{E}-03$ & - & 14.4 & -54 & 36 & - \\
\hline $80 \mathrm{R}-7,23$ & 433.23 & 2 & 7.49E-03 & 4.09E-03 & 4 & 4.8 & -65 & 52 & 338 \\
\hline $81 \mathrm{R}-2,54$ & 435.54 & 2 & $2.30 \mathrm{E}-04$ & $2.23 \mathrm{E}-04$ & 2 & 2.7 & -68 & 71 & 239 \\
\hline $81 \mathrm{R}-3,37$ & 436.64 & 1,9 & $3.56 \mathrm{E}-04$ & $2.12 \mathrm{E}-04$ & 4 & 4.4 & -55 & 59 & 245 \\
\hline $81 \mathrm{R}-7,64$ & 442.84 & 2 & $1.45 \mathrm{E}-04$ & $1.26 \mathrm{E}-04$ & 17 & 3.0 & -71 & - & - \\
\hline $82 \mathrm{R}-1,18$ & 443.18 & 2,9 & $6.06 \mathrm{E}-02$ & $3.31 \mathrm{E}-03$ & 14 & 48.2 & 62 & 80 & 22 \\
\hline $82 \mathrm{R}-2,13$ & 444.63 & 5 & $2.23 \mathrm{E}-04$ & $1.76 \mathrm{E}-04$ & 3 & 3.3 & -72 & 53 & 211 \\
\hline $82 R-5,45$ & 449.02 & 2,8 & $1.40 \mathrm{E}-02$ & $8.96 \mathrm{E}-03$ & - & 4.1 & -71 & - & - \\
\hline $82 R-6,11$ & 450.61 & 3,8 & $3.41 \mathrm{E}-02$ & - & 4 & - & -70 & 33 & 233 \\
\hline $83 R-2,105$ & 455.05 & 2,10 & $7.55 \mathrm{E}-04$ & $5.64 \mathrm{E}-04$ & 61 & 3.5 & 38 & 63 & 319 \\
\hline $83 R-4,95$ & 457.95 & 1,9 & $2.48 \mathrm{E}-04$ & $1.78 \mathrm{E}-04$ & 3 & 3.7 & -57 & 49 & 126 \\
\hline $83 \mathrm{R}-7,104$ & 462.54 & 5,8 & 2.89E-04 & $3.30 \mathrm{E}-04$ & 4 & 2.3 & -55 & 63 & 48 \\
\hline $84 R-2,67$ & 464.17 & 2,10 & $1.33 \mathrm{E}-03$ & $5.08 \mathrm{E}-04$ & 2 & 6.9 & -72 & 78 & 198 \\
\hline $84 \mathrm{R}-3,14$ & 465.14 & 2 & $5.96 \mathrm{E}-04$ & $8.85 \mathrm{E}-04$ & 35 & 1.8 & -46 & 72 & 183 \\
\hline $84 \mathrm{R}-4,23$ & 466.46 & 2 & $6.51 \mathrm{E}-04$ & $1.15 \mathrm{E}-03$ & 58 & 1.5 & -25 & 65 & 141 \\
\hline $84 \mathrm{R}-7,3$ & 470.14 & 2,8 & $1.25 \mathrm{E}-03$ & $6.17 \mathrm{E}-04$ & 40 & 5.3 & 44 & 73 & 90 \\
\hline $85 \mathrm{R}-1,75$ & 472.25 & 2,9 & $5.96 \mathrm{E}-03$ & $1.88 \mathrm{E}-03$ & 3 & 8.3 & -60 & 71 & 329 \\
\hline $85 \mathrm{R}-3,94$ & 475.39 & 2,9 & $3.71 \mathrm{E}-03$ & $2.24 \mathrm{E}-03$ & 4 & 4.3 & -60 & 60 & 30 \\
\hline $85 R-4,9$ & 476.09 & 1,9 & $1.48 \mathrm{E}-03$ & $1.02 \mathrm{E}-03$ & 2 & 3.8 & -68 & 80 & 128 \\
\hline $85 R-4,37$ & 476.11 & 1,9 & $3.08 \mathrm{E}-04$ & $6.01 \mathrm{E}-04$ & 57 & 1.3 & 49 & 80 & 349 \\
\hline $85 \mathrm{R}-7,17$ & 480.67 & 5 & $1.11 \mathrm{E}-03$ & $5.35 \mathrm{E}-04$ & 2 & 5.5 & -74 & 73 & 224 \\
\hline $86 \mathrm{R}-1,24$ & 481.24 & 1,8 & $9.94 \mathrm{E}-04$ & $6.03 \mathrm{E}-04$ & 2 & 4.3 & 33 & 47 & 45 \\
\hline $86 \mathrm{R}-3,79$ & 484.79 & 2,9 & $1.24 \mathrm{E}-02$ & $6.34 \mathrm{E}-03$ & 3 & 5.2 & -82 & 80 & 175 \\
\hline $86 \mathrm{R}-3,133$ & 485.33 & 2,9 & $7.84 \mathrm{E}-03$ & 2.75E-03 & 4 & 7.5 & -36 & 66 & 150 \\
\hline $86 \mathrm{R}-6,143$ & 489.93 & 2,9 & $1.22 \mathrm{E}-02$ & - & 4 & - & -64 & 69 & 189 \\
\hline $87 \mathrm{R}-3,64$ & 493.98 & 2,9 & $4.85 \mathrm{E}-04$ & $5.09 \mathrm{E}-04$ & 68 & 2.5 & -12 & 69 & 241 \\
\hline $87 \mathrm{R}-3,115$ & 494.49 & 2,8 & 8.91E-04 & $4.97 \mathrm{E}-04$ & 5 & 5.4 & -70 & 76 & 86 \\
\hline $87 \mathrm{R}-5,11$ & 496.61 & 1,9 & $1.52 \mathrm{E}-02$ & $8.82 \mathrm{E}-03$ & 3 & 4.5 & -59 & 59 & 121 \\
\hline $87 R-5,20$ & 496.70 & 1,9 & $1.65 \mathrm{E}-02$ & - & 6 & - & -79 & 70 & 188 \\
\hline $87 \mathrm{R}-6,28$ & 498.12 & 2,9 & $4.87 \mathrm{E}-03$ & $5.31 \mathrm{E}-03$ & 3 & 2.4 & -77 & 61 & 225 \\
\hline
\end{tabular}

$J_{n r m}=$ the NRM intensity; $K=$ the initial magnetic susceptibility; MDF = the median demagnetizing field; and $Q=$ the Koenigsberger ratio. $I_{n r m}=$ the NRM inclination in degrees. $I_{s}$ is the stable inclination in degrees, and $D_{s}$ is the uncorrected, stable declination in degrees. Rock types are based upon Robinson, Von Herzen, et al. (1989): (1) gabbro, (2) olivine gabbro, (3) Fe-Ti gabbro, (4) norite, (5) troctolite, (6) microgabbro, (7) basalt, (8) altered sample, (9) deformed and/or metamorphosed sample, (10) sample including a contact between two rock types or distinctive textures.

\section{Koenigsberger Ratio $\left(Q_{n}\right)$}

The Koenigsberger ratio, $Q_{n}=J_{n r m} / K H$, can be calculated using the NRM intensity and the initial susceptibility. $H=0.38 \mathrm{Oe}$, the value of the ambient geomagnetic field at Site 735 (Merrill and McElhinny, 1983), was used for our calculations. The Koenigsberger ratio is an estimate of the relative contributions of remanent and induced magnetization within a given rock. It is commonly used to determine whether the in-situ magnetization is dominated by remanent magnetization $\left(Q_{n}>1\right)$ or an induced component parallel to the current field $\left(Q_{n}<1\right)$. As discussed later, many of the magnetite- and ilmenite-rich gabbros have a strong secondary component that dominates NRM and is probably acquired during drilling. $Q_{n}$ values calculated from these gabbros may indicate the relative importance of the secondary component to induced magnetization. Therefore, care should be taken when considering $Q_{n}$ values calculated from $\mathrm{Fe}-\mathrm{Ti}$ oxide rich gabbros (especially, unit VI). However, as shown in the later section, the overall distribution of the $Q_{n}$ ratio obtained by excluding those gabbros is not so different from that typically observed.

The Koenigsberger ratio calculated ranged between 0.1 and 58.4. Overall variation of the Koenigsberger ratio with depth is shown in Figure 4. The range of the Koenigsberger ratio is comparable to those of other oceanic gabbros (Fox and Opdyke, 1973; Pariso and Johnson, 1989b). Figure 4 and Table 1 show that most of the $Q_{n}$ values lie between 1 and 10 , and only 10 samples out of 245 calculations have $Q_{n}$ values of less than unity. This indicates that the magnetic remanences 
NRM Inclination

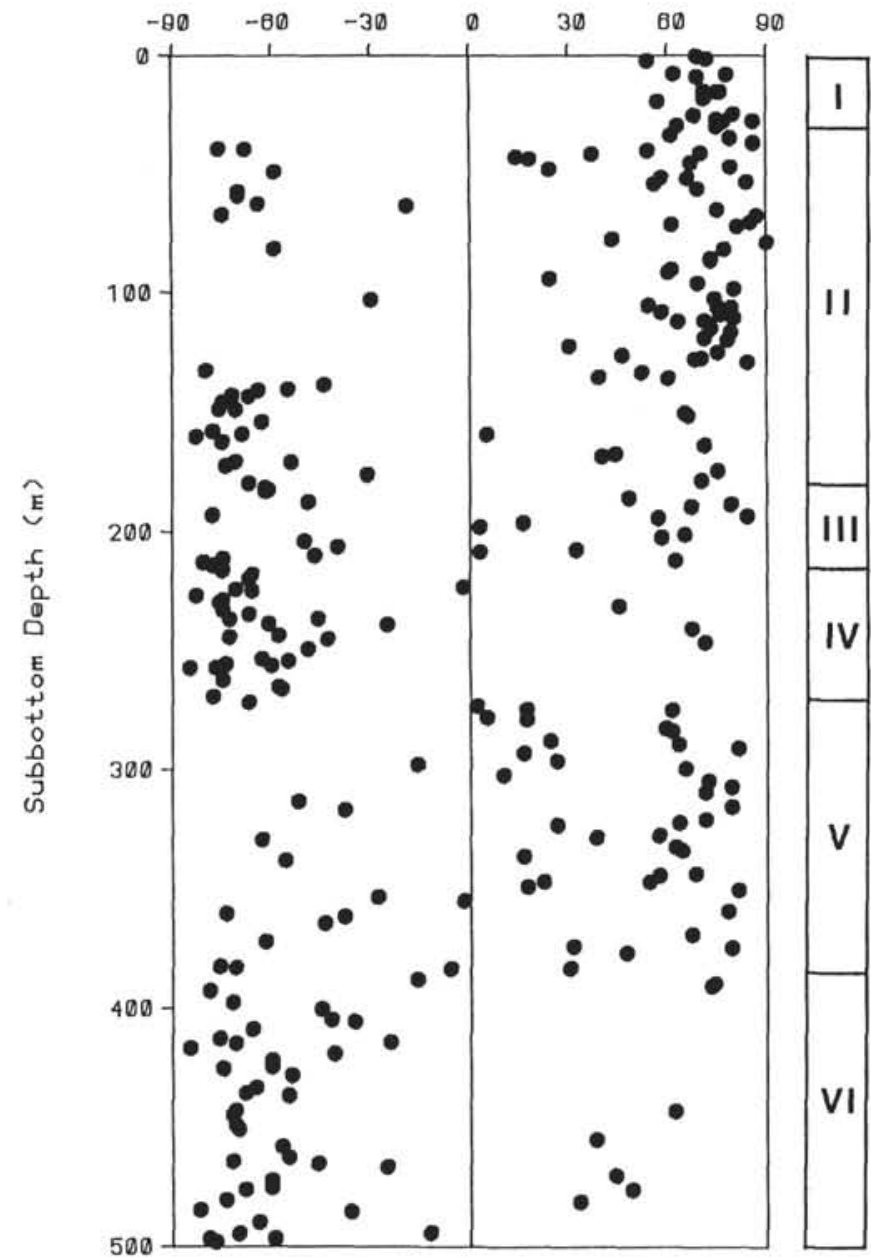

Figure 2. Plot of NRM inclinations vs. depth for Hole 735B.

measured were not disturbed by magnetizations induced by the present geomagnetic field.

\section{Alternating-Field and Thermal Demagnetizations}

A total of 262 samples out of 264 were progressively demagnetized, either by the alternating-field $(\mathrm{AF})$ or the thermal method to obtain reliable paleomagnetic directions and to observe magnetic behavior of the samples during the demagnetization. The peak $\mathrm{AF}$ at which half of the original remanence is demagnetized (MDF) was determined from demagnetization curves plotted for all samples subjected to $\mathrm{AF}$ demagnetization. The MDF is a good parameter that characterizes the stability of natural remanence. The amount of angular change in the direction of remanence during $\mathrm{AF}$ demagnetization is well described by the MDF of the sample. Samples having higher MDF values show a smaller angular change in remanence. Figure 5 is a plot of MDF vs. subbottom depth in Hole 735B. As Figure 5 shows, the majority of the recovered samples have high MDF values $(>15 \mathrm{mT})$. However, many of samples from Fe-Ti oxide gabbros and the magnetite- and ilmenite-rich olivine gabbros have low MDF values $(<5 \mathrm{mT})$.

Concerning the directional changes during AF demagnetizations, there are two types. The most frequently observed type in Figure $6 \mathrm{~A}$ is characterized by the gradual removal of a

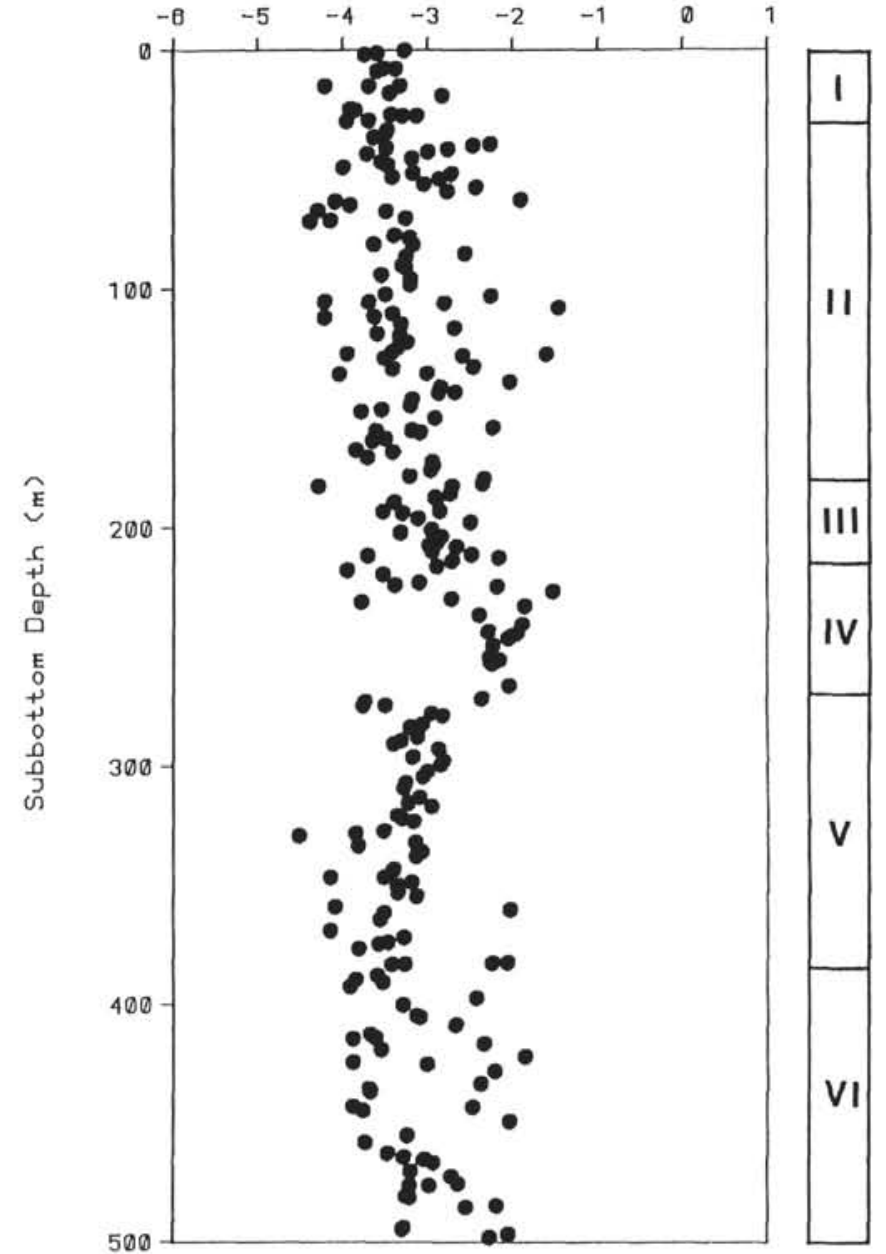

Figure 3. Plot of initial magnetic susceptibility vs. depth in Hole 735B.

single, stable magnetic vector. As shown in Figure 6A, many of this type indicate very slight removal of an unstable component at lower demagnetization steps. This unstable component is probably the same as that observed in Fe-Ti oxide gabbros, which is discussed later. Figure 6B depicts another type of magnetic vector change during AF demagnetizations that is most commonly observed in gabbros containing extremely large amounts of $\mathrm{Fe}-\mathrm{Ti}$ oxide minerals. This type of demagnetization exhibits a dramatic change in the remanence direction and a rapid decrease in intensity during lower steps of AF demagnetization, followed by the appearance of a stable remanence component in the higher demagnetization steps. This means that samples showing this type of change have unstable NRMs and low MDFs. These findings are consistent with the MDF values observed earlier. In many cases, stable components appear after NRMs of the samples lose more than $95 \%$ of their magnetization. Although reversals of magnetic inclination are a characteristic feature in most cases of this type, the change in magnetic declination is not always so large as that shown in Figure $6 \mathrm{~B}$, indicating that secondary (unstable) components of samples are not aligned antipodally to stable components. The secondary components have normal (negative) polarity, and their horizontal components seem to be acquired in random directions with respect to the declinations of the stable components. 


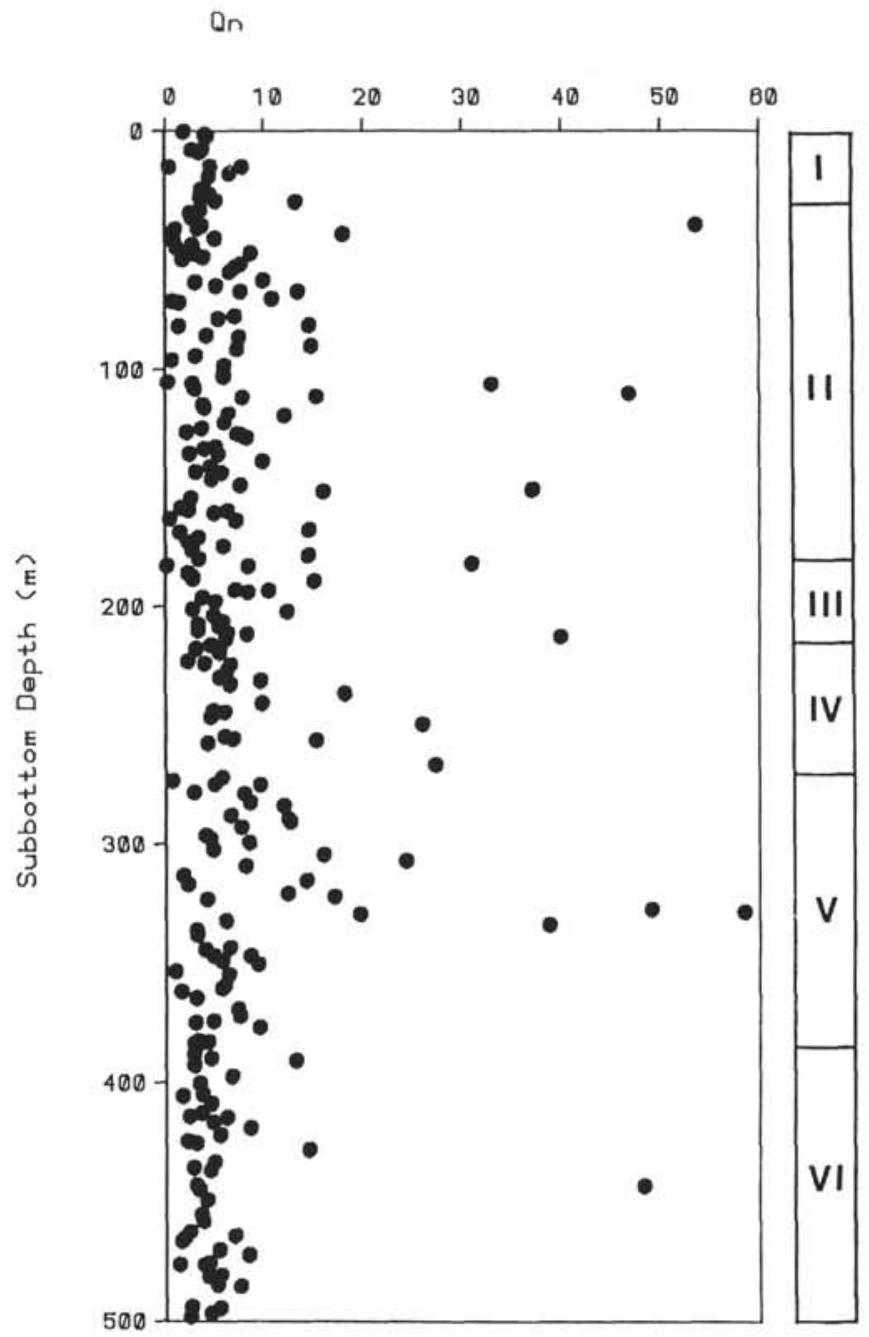

Figure 4. Plot of the Koenigsberger ratio vs. depth in Hole 735B, using a value of 0.38 Oe for the magnetic field intensity at Site 735 .

Six shore-based progressive thermal demagnetizations were performed to supplement the shipboard thermal demagnetization data. However, stable inclinations were obtained from only two thermal demagnetizations, resulting in five of 10 determinations of stable inclinations. All of the four thermal demagnetizations performed on the samples containing fairly large amounts of $\mathrm{Fe}-\mathrm{Ti}$ oxide minerals were unsuccessful. Figure 7 is a typical example of successful thermal demagnetization data obtained from four samples, including two shorebased determinations of good data. Figure 7 shows that the remanence of the sample is clearly dominated by a stable component carried by a magnetic mineral of high blocking temperature $\left(560^{\circ}\right.$ to $\left.580^{\circ} \mathrm{C}\right)$, such as magnetite. This is consistent with previous studies (Kent et al., 1978; Dunlop and Prevot, 1982).

\section{Stable Inclination $\left(I_{s}\right)$}

A plot of stable inclinations (determined from a leastsquares approximation using Zijderveld diagrams obtained for progressive demagnetization data) vs. depth is shown in Figure 8 . In contrast to NRM inclinations that indicate mixed magnetic polarities (Fig. 2), Figure 8 shows all but three samples have positive (reversed) stable inclinations. To examine whether the two negative stable inclinations $\left(-64^{\circ}\right.$ from

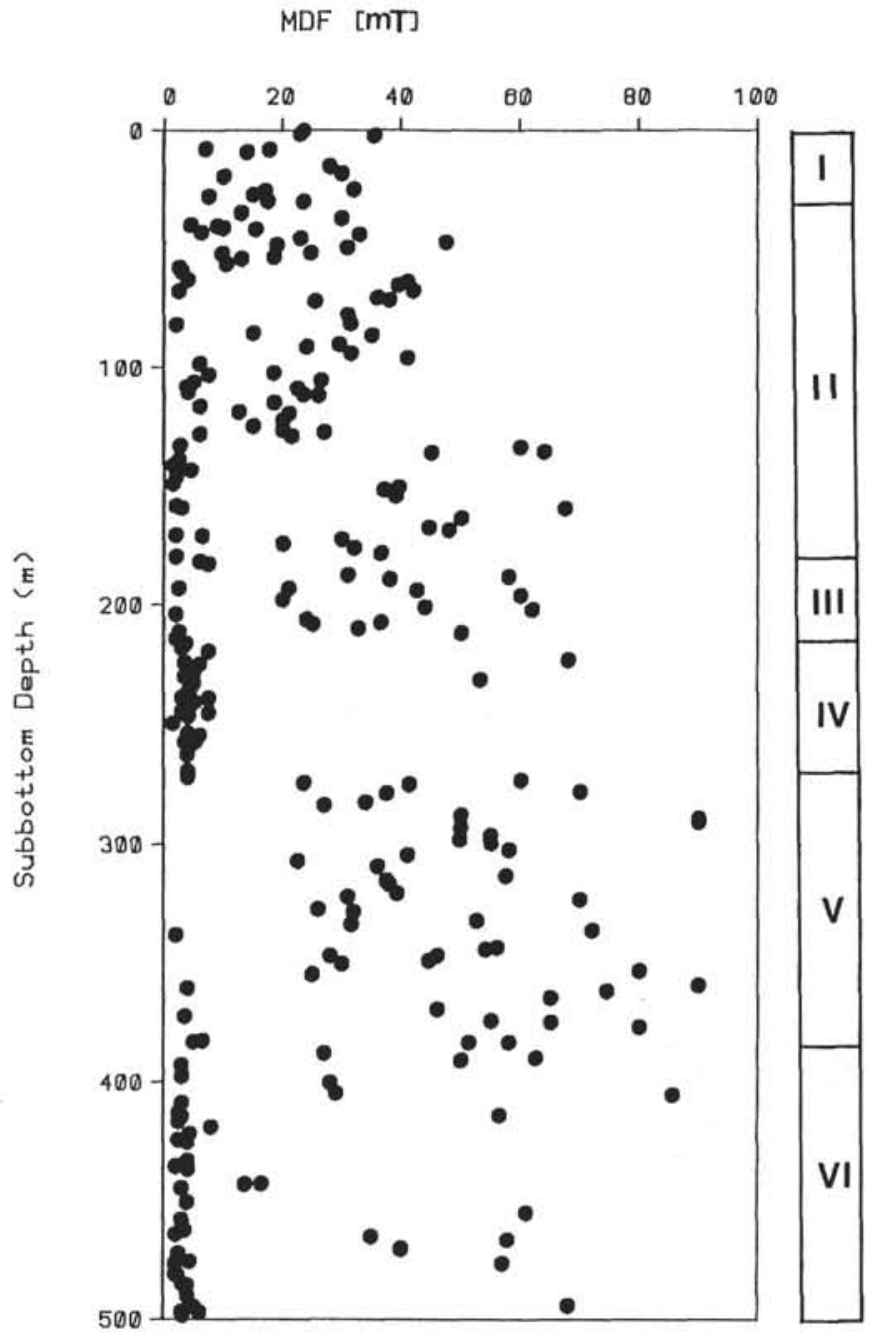

Figure 5. Plot of median demagnetizing field vs. depth in Hole 735B.

Sample 118-735B-38R-4, $28 \mathrm{~cm}$, and $-68^{\circ}$ from Sample 118$735 \mathrm{~B}-58 \mathrm{R}-2,33 \mathrm{~cm}$ ) were caused by misorientation, the cores were observed carefully on board the JR, indicating that it was unclear if the two samples were or were not incorrectly oriented. However, considering the general tendency observed in Figure 8 and the absolute values of the two positive inclinations, which are similar to those of the majority, one might conclude that these two samples were probably oriented incorrectly, although the latter conclusion may be a good reason for concluding the opposite. Concerning the negative inclination value of $-3^{\circ}$ calculated from Sample 118-735B$22 \mathrm{R}-1,2 \mathrm{~cm}$, this sample was not identified and so must be examined later. We think that this sample was probably rotated during drilling. Based upon McFadden and Reid's method (1982), average inclination was calculated as $66^{\circ} \pm 5^{\circ}$, leaving out the three negative stable inclinations. The geocentric axial dipole field for site $735\left(33^{\circ} \mathrm{S}\right)$ is $-52^{\circ}$, showing that the average of these stable inclinations is not only reversed but also slightly steeper. A marine magnetic anomaly survey suggests an age of about $12 \mathrm{Ma}$ (anomaly 5A) for the study area (Dick et al., 1988). The reversed magnetization obtained from Hole $735 \mathrm{~B}$ may be correlated to one of the reversed polarity chrons observed around anomaly $5 \mathrm{~A}$. Note that the logging hole inclines $4^{\circ}$ to $6^{\circ}$ northward from the vertical axis. If this is the case, one would expect a slight shallowing of the 
observed inclination. This means that the true inclination becomes slightly steeper than the average value calculated. Possible causes of this slightly steeper inclination are paleosecular variation and tectonic rotation. We feel that tectonic rotation is more likely because to cool the whole drilled section probably required a long enough time to average the secular variations.

\section{DISCUSSION}

\section{Secondary Component of Recovered Samples and Its Origin}

Although all of the stable inclinations show reversely magnetized inclinations, NRM inclinations calculated from the recovered gabbros have both normal and reversed polarities, as shown in Figure 2. If NRM inclinations represent in-situ magnetization, effective magnetization intensities responsible for seafloor spreading magnetic anomalies should be lower than those expected from NRM intensities. To summarize, $\mathrm{Fe}-\mathrm{Ti}$ oxide gabbros and gabbros that contain extremely large amounts of Fe-TI oxide minerals indicate field reversals during AF-demagnetizations, higher NRM intensities, and unstable secondary components of a positive inclination polarity. As mentioned previously, this is defined by a rapid decrease in intensity and a dramatic change of the remanent magnetization direction during lower steps of AF-demagnetization, followed by the appearance of a stable component in the higher demagnetization steps. In other words, these gabbros are characterized by the presence of a very strong, unstable secondary component that dominates the NRM in intensity and direction.

Microscopic observation showed that these Fe-Ti oxiderich gabbros generally have the intergrowths of coarse-grained ilmenite and magnetite. But there is other magnetite in the gabbros that is typically much finer-grained, less reflective, and in vermicular to skeletal morphologies (Shipboard Scientific Party, 1989). It is evident that this finer-grained magnetite carries the stable component of natural remanent magnetization in these gabbros and the coarse-grained magnetite gives the secondary magnetization.

To determine if these normally magnetized NRMs (mostly composed of the secondary component stated above) are in-situ, logging magnetic inclination data were used. Figure 9 (Pariso et al., this volume; Robinson, Von Herzen, et al., 1989) shows magnetic inclinations computed from a fully oriented, three-component, downhole magnetometer. Magnetic inclinations observed within a hole may be calculated using magnetic data obtained from recovered rocks. Therefore, it is possible to estimate the validity of whether NRMs of $\mathrm{Fe}$-Ti oxide gabbros are in-situ by comparing magnetic inclinations observed by magnetic logging with those that are calculated.

The magnetic field within a hole consists of two components: one is the ambient geomagnetic field and the other is the magnetic field related to the surrounding magnetized material. Both the remanent and induced magnetizations may contribute to the magnetic field observed within a hole. Koenigsberger ratios calculated from the studied gabbros are sufficiently larger than unity, indicating that relative importance of remanent magnetization on induced magnetization can be ignored. Let $F_{o}$ and $I_{o}$ be the total force of the ambient geomagnetic field and the inclination, respectively. The geomagnetic field can then be expressed as

$$
\begin{aligned}
& H=F_{o} \cos I_{o}, \\
& Z=F_{o} \sin I_{o},
\end{aligned}
$$

By taking the case where the material surrounding the hole is homogeneously magnetized and the shape of the hole is a perfect circle, the magnetic field caused by the surrounding material at the center of the hole is calculated as

$$
h=2 \pi M \cos I,
$$

$$
z=-4 \pi \sin I
$$

where $M$ is intensity and $I$ is inclination of the magnetization of the surrounding material (Bosum and Eberle, 1983). By adding Equations 1 and 2, the inclination of the magnetic field within the hole can be estimated as

$$
\tan I_{o b s}=(Z+z) /(H+h) .
$$

The total geomagnetic force at the position of Hole 735B is $0.38 \mathrm{Oe}$ and the inclination is $-60^{\circ}$ (IGRF, 1980). For example, when taking $54^{\circ}$ and $4.8 \mathrm{~A} / \mathrm{m}$ as values for $I$ and $M$, respectively, $I_{o b s}$ is calculated as $-65.6^{\circ}$, using Equations 1, 2, and 3.

Table 2 lists and Figure 10 presents the results obtained by using inclinations and magnetization intensity values for NRM values of selected samples. Magnetic field inclinations expected from stable remanences of surrounding rocks (recovered samples, $I_{c s}$ ) are also presented (Fig. 11, Table 2). When calculating $I_{c s}$ values, stable remanent inclination $\left(I_{s}\right)$ and magnetic remanence intensity values for stable magnetization $\left(J_{s t}\right)$ were taken. To estimate the latter $\left(J_{s t}\right)$, magnetization intensities at demagnetization steps when only stable components predominate were used. Consequently, $J_{s t}$ values are minimum estimations for the stable magnetization. The obtained average intensity for $J_{s t}$ is 1.6 $\mathrm{A} / \mathrm{m}$, a relatively high value. Comparison of Figure 9 with Figure 10 clearly reveals disagreement, indicating that there must be some doubts about whether or not NRM values of the magnetite- and ilmenite-rich samples are in-situ. In contrast, Figures 9 and 11 agree well with each other, suggesting that in-situ magnetizations of $\mathrm{Fe}$-Ti oxide rich samples differ from observed NRM values, but are probably nearer those of stable magnetizations. This suggests that the secondary components dominating the NRMs of the magnetite- and ilmenite-rich samples were probably acquired during drilling processes.

Concerning the acquisition mechanism of this secondary magnetizations several possibilities come to mind. Possible mechanisms are isothermal remanent magnetization (IRM), viscous remanent magnetization (VRM), piezo remanent magnetization (PRM), partial thermo remanent magnetization (PTRM) and chemical remanent magnetization (CRM). As suggested previously, the secondary magnetization was caused by drilling-induced remanent magnetization (DIRM), and VRM, PTRM, and CRM have lower priority because their acquisition mechanisms require a longer time than DIRM. However, in the following discussions, we will take them into consideration because these three types of remanence are commonly acquired as a secondary component. Several laboratory experiments and considerations were conducted to examine the possible acquisition mechanism, although the irreversibility of the acquisition environment makes it difficult to determine the cause.

Figure 12 is the result of AF-demagnetization of the thermoremanent magnetization applied to Sample 118-735B$47 \mathrm{R}-2,111 \mathrm{~cm}$, in a field of $0.5 \mathrm{Oe}$ with heating up to $600^{\circ} \mathrm{C}$. This experiment was performed to examine whether PTRM 


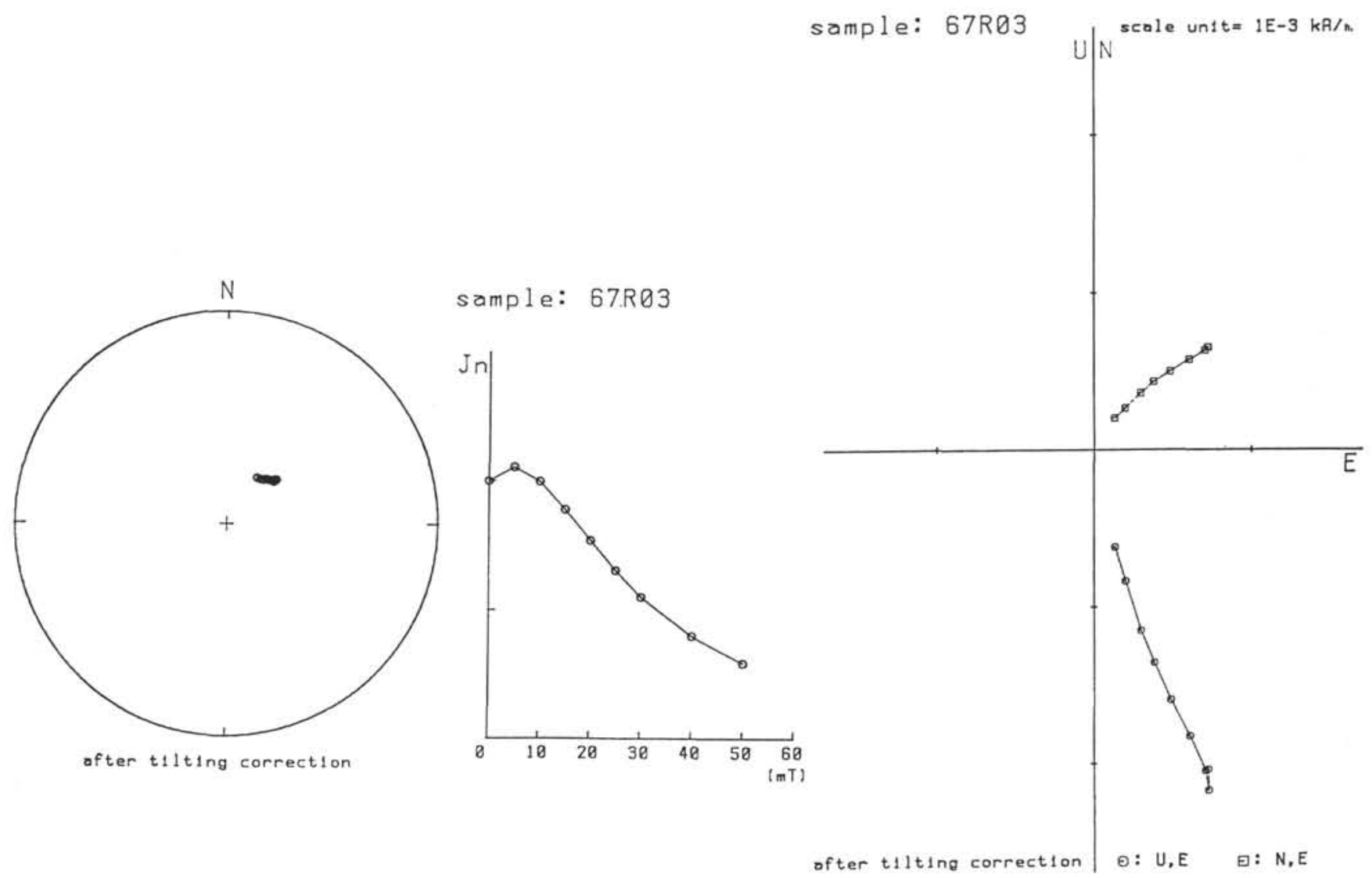

Figure 6. Plot of AF demagnetization data in three diagrams. A. Typical example of stable remanence data (Sample $118-735 \mathrm{~B}-67 \mathrm{R}-3,90 \mathrm{~cm})$. The Zijderveld diagram on the right shows no change in magnetic direction during demagnetization, as does the stereographic projection of the total intensity vector on the left. The diagram in the middle is the decay of normalized intensity with increasing peak alternating field. B. Typical example of data including very unstable and strong secondary component (Sample 118-735B-47R-2, 111 cm). Although the Zijderveld diagram on the right shows only a large secondary component of magnetization, the stereographic projection of the total intensity vector on the left obviously indicates the dramatic change in direction (field reversals) at lower steps and good grouping at higher steps. In the middle of the diagram, an abrupt decay in normalized intensity can be seen to occur at low-peak alternating fields.

(or CRM) could be the cause, although chemical changes of magnetic minerals during the thermal treatment, such as oxidation, might contribute to effects on the magnetic properties of the sample. As Figure 12 indicates, the applied thermal remanence direction was very stable, which is not consistent with the characteristics of the secondary component mentioned above and agrees with the general property of the thermoremanent magnetization. This makes us think that PTRM (and thus CRM) may not be the cause of the unstable magnetization.

VRM and PRM probably are not the causes because in most cases AF-demagnetization data show that the secondary components of the studied samples are not aligned in antipodes with stable components (Fig. 6B), which means that these secondary components were not acquired in the direction parallel to the external field (which is nearly the ambient geomagnetic field shown in Fig. 9). Figure 13 exhibits VRM acquisition data during six-week storage tests in a magnetic field of 0.5 Oe obtained from Sample 118-735B-80R-3, $124 \mathrm{~cm}$, after AF-demagnetization. VRM acquisition during the Brunhes epoch $(0.7 \mathrm{~m} . \mathrm{y}$.; calculated using the viscosity coefficient obtained from Fig. 13) was estimated to be $1.5 \mathrm{~A} / \mathrm{m}$. This is doubtlessly underestimated because AF-demagnetization usually causes a dramatic decrease in the ability to acquire VRM, compared to the same sample with an undemagnetized ther- mal remanent magnetization (Tivey and Johnson, 1984). However, even considering this fact, the estimated VRM acquisition value is significantly lower than the NRM of $32.7 \mathrm{~A} / \mathrm{m}$ (about $1 / 20$ ). Considering that the secondary magnetic component is not aligned antipodally with the stable component and the low VRM acquisition of the sample, we conclude that VRM is not the cause either.

Audunsson and Levi (1989) showed that IRM explains the observed DIRM well and concluded that the DIRM in the drill core is most easily explained as having been produced during the initial drilling by a strong non-uniform field concentrated near the cutting rim of the drill string. As generally observed in the drill core, DIRM in the present samples adds a vertical component. During Leg 117, immediately after core recovering, very strong magnetization of drilling bits and pipes was observed (Niitsuma, pers. comm., 1988). This would suggest significant contribution of IRM caused by drilling tools to the observed secondary component.

\section{Magnetic Properties Based Upon the Degree of Metamorphism/Alteration}

Concerning the mean value of NRM intensity for gabbro samples measured, it has been reported that essentially no difference exists between metamorphosed and unmetamor- 


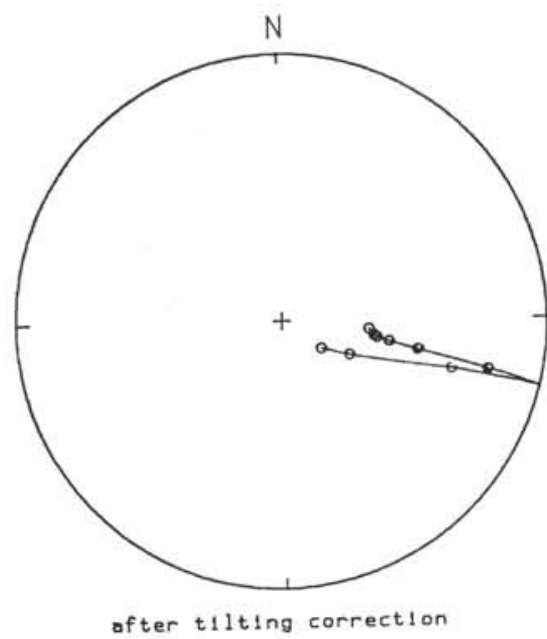

Figure 6 (continued).

sample: $47 R 02$
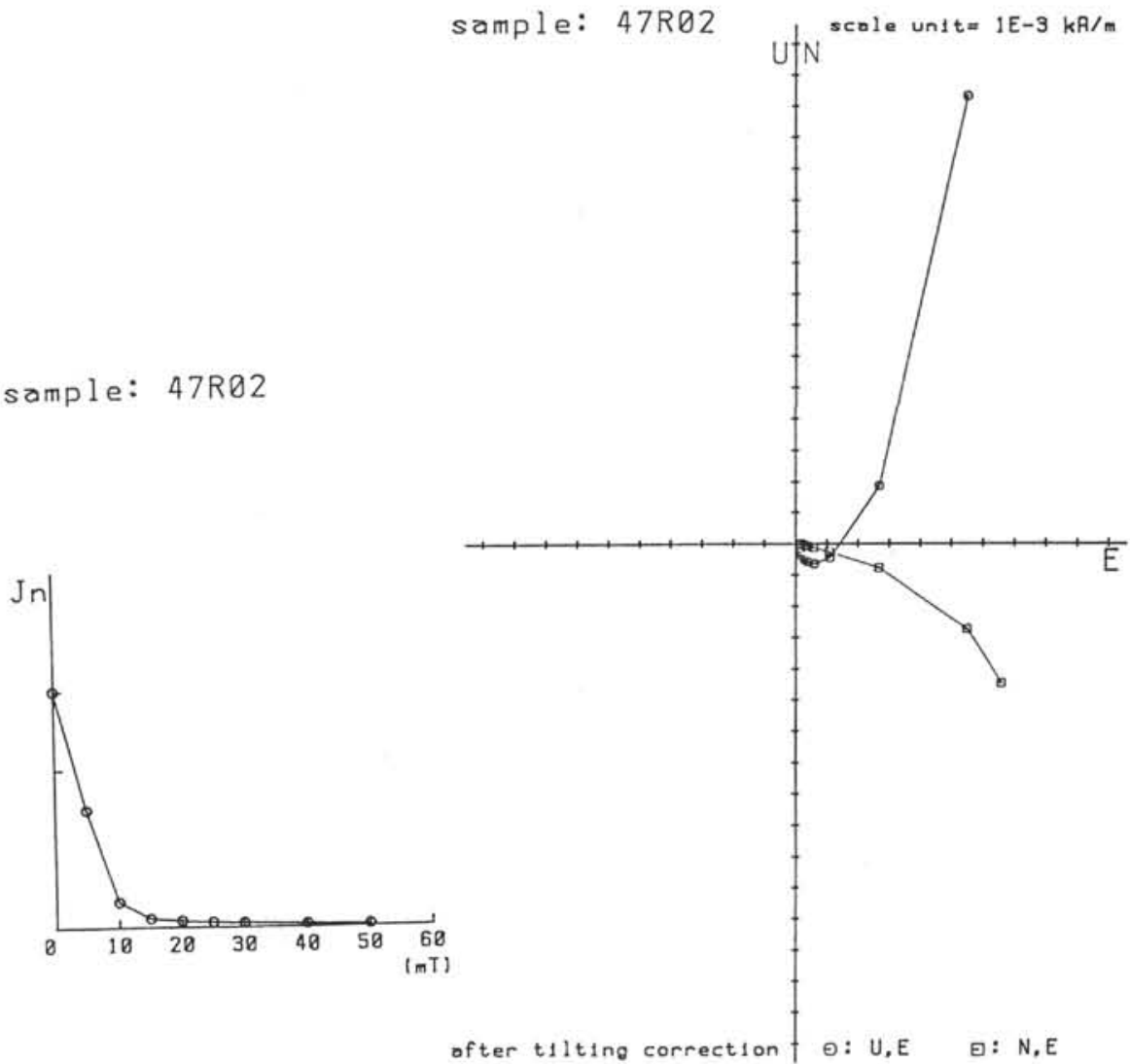

phosed gabbros (Fox and Opdyke, 1973; Kent et al., 1978; Dunlop and Prevot, 1982). To examine this hypothesis, magnetic properties based upon the degree of metamorphism/ alteration are observed. With increasing degree of metamorphism/alteration, primary anhydrous phases are altered and replaced with hydrous phases such as chlorite, hornblende, amphibole, and so on. Here, a summation of percentages of secondary hydrous minerals (clay, chlorite, hornblende, amphibole, talc, epidote, tremolite, and actinolite) calculated on the basis of the thin-section descriptions made for Leg 118 samples (Shipboard Scientific Party, 1989) was used to classify the degree of metamorphism/alteration, rather than the commonly used temperature-dependent definition of the metamorphic grade. This was done because we think that this is a good parameter for indicating the effects of pressure-temperature times the period during which samples suffered metamorphism or alteration (Ozawa, Urabe, pers. comm., 1989). Table 3 lists and Figure 14 presents the results of calculations for all of the studied samples having both magnetic measurements and thin section descriptions. On the basis of the results of the calculations shown in Figure 14, the degree of metamorphism/alteration has been divided into three categories: high, medium, and low. Samples belonging to the low grade have total amounts of secondary hydrous minerals of less than $10 \%$. Samples that include $10 \%$ to $25 \%$ and more than $25 \%$ of secondary hydrous minerals are defined as medium and high grades, respectively.

Figure 15 shows a distribution of NRM intensity. Because all of the samples having negative (normal) NRM inclinations acquired significant amounts of secondary components during drilling, such samples have been left out of the discussion.
Therefore, the number of the samples was not large enough to determine the detailed variations of NRM intensities; thus, we mainly discuss here the overall observation for each grade. NRM intensities for low-grade samples ranged from 0.097 to $21.0 \mathrm{~A} / \mathrm{m}$, a range of about two orders of magnitude. An arithmetic mean value of $2.50 \mathrm{~A} / \mathrm{m}$ (indicated by a solid triangle in the lower part of Fig. 15) was obtained for all the low-grade samples. This value was reduced to $1.41 \mathrm{~A} / \mathrm{m}$ (shown by an open triangle in the lower part of Fig. 15) by leaving out the samples showing the highest magnetization (Sample 118-735B-54R-5, $117 \mathrm{~cm}$ ) from the mean calculation described above. Medium-grade samples show a wider variation of magnetizations ranging between 0.0049 and 7.24 $\mathrm{A} / \mathrm{m}$, giving an arithmetic mean value of $1.19 \mathrm{~A} / \mathrm{m}$ (a solid triangle in the middle part of Fig. 15). The widest variation of $\mathrm{NRM}$ intensities (ranging from 0.0087 to $37.2 \mathrm{~A} / \mathrm{m}$ ) was observed in the high grade samples. The arithmetic mean was calculated as $3.76 \mathrm{~A} / \mathrm{m}$ (a solid triangle in the upper part of Fig. 15), and this value was also reduced to $1.61 \mathrm{~A} / \mathrm{m}$ (an open triangle in the upper part of Fig. 15) by taking out two samples having magnetizations higher than $10 \mathrm{~A} / \mathrm{m}$ from the calculations.

All of the average means calculated after excluding the highest sample values are not so different among the three studied metamorphism/alteration grades, but are higher than previously reported mean values ranging from 0.48 to 0.89 $\mathrm{A} / \mathrm{m}$ (Hayling and Harrison, 1986). Figure 15 also indicates that the distribution of magnetizations for the three grades of samples are similar, with large overlaps in range, indicating that essentially no difference exists between the three grades, as suggested by the previous studies. However, one might 


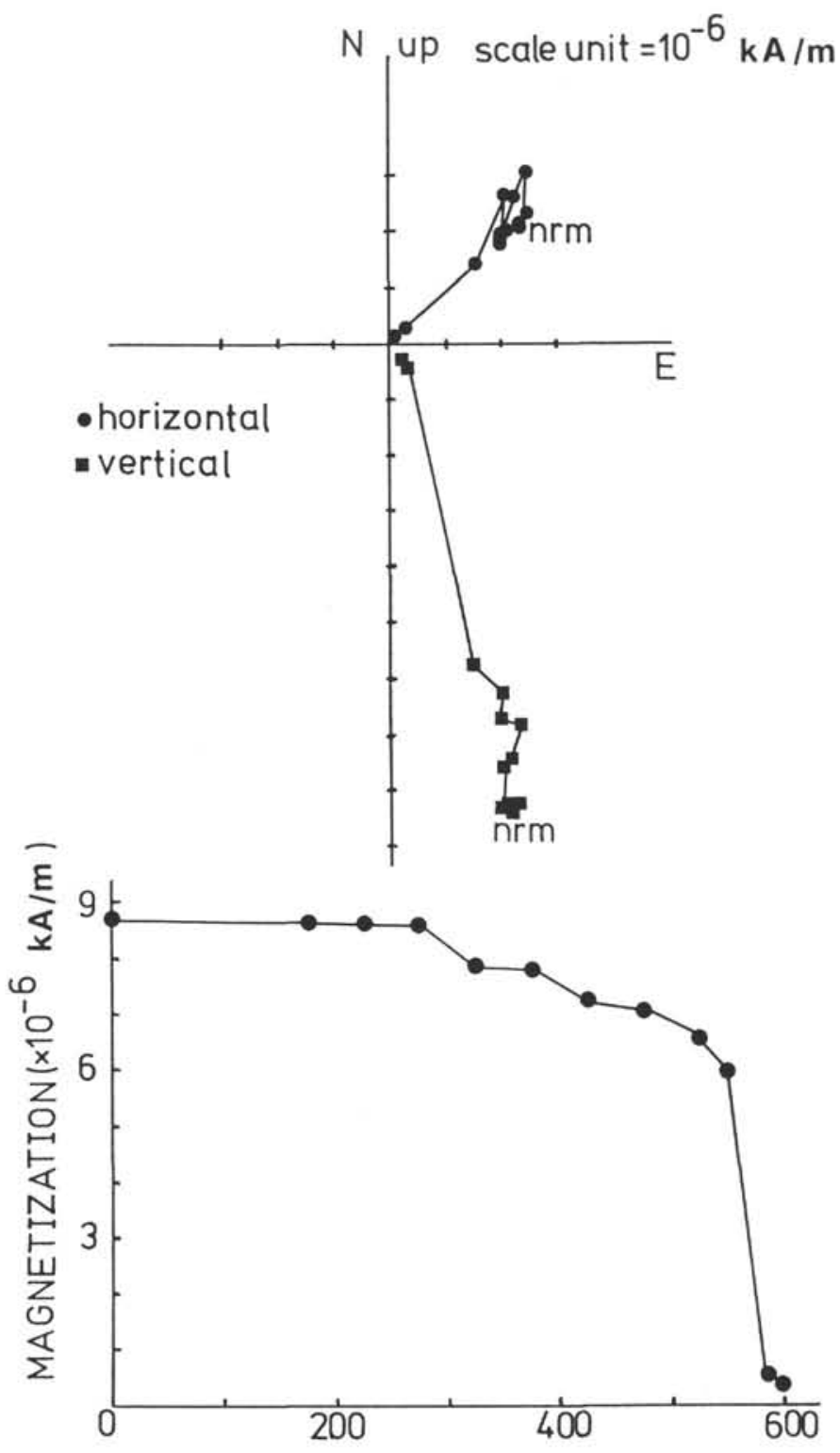

Figure 7. Plot of typical example of thermal demagnetization data in two diagrams for Sample 118-735B-3D-1, $58 \mathrm{~cm}$. Several weakly defined components appear on the Zijderveld plot above; however, the total magnetic vector is dominated by the stable component carried by a magnetic mineral of high blocking temperature (560 to $\left.580^{\circ} \mathrm{C}\right)$.

note that samples of medium and high grades have relatively higher frequencies at the lower range of values of NRM intensity (Fig. 15).

Figure 16 shows the distribution of susceptibility values observed for all of the studied samples having susceptibility measurements. Low-grade samples have susceptibility values that range from $7.05 \times 10^{-5}$ to $5.61 \times 10^{-3} \mathrm{cgs}$, with an arithmetic mean value of $1.12 \times 10^{-3} \mathrm{cgs}$. (noted as a solid triangle in the lower part of Fig. 16). The initial magnetic susceptibility of medium-grade samples ranges from $3.40 \times$ $10^{-5}$ to $1.10 \times 10^{-2} \mathrm{cgs}$. The arithmetic mean value was calculated as $1.54 \times 10^{-3} \mathrm{cgs}$ (a solid triangle of the middle part of Fig. 16). By leaving out the sample having the highest value (Sample 118-735B-51R-1, $102 \mathrm{~cm}$ ), the mean calculation becomes $1.31 \times 10^{-3} \mathrm{cgs}$ (an open triangle of the middle

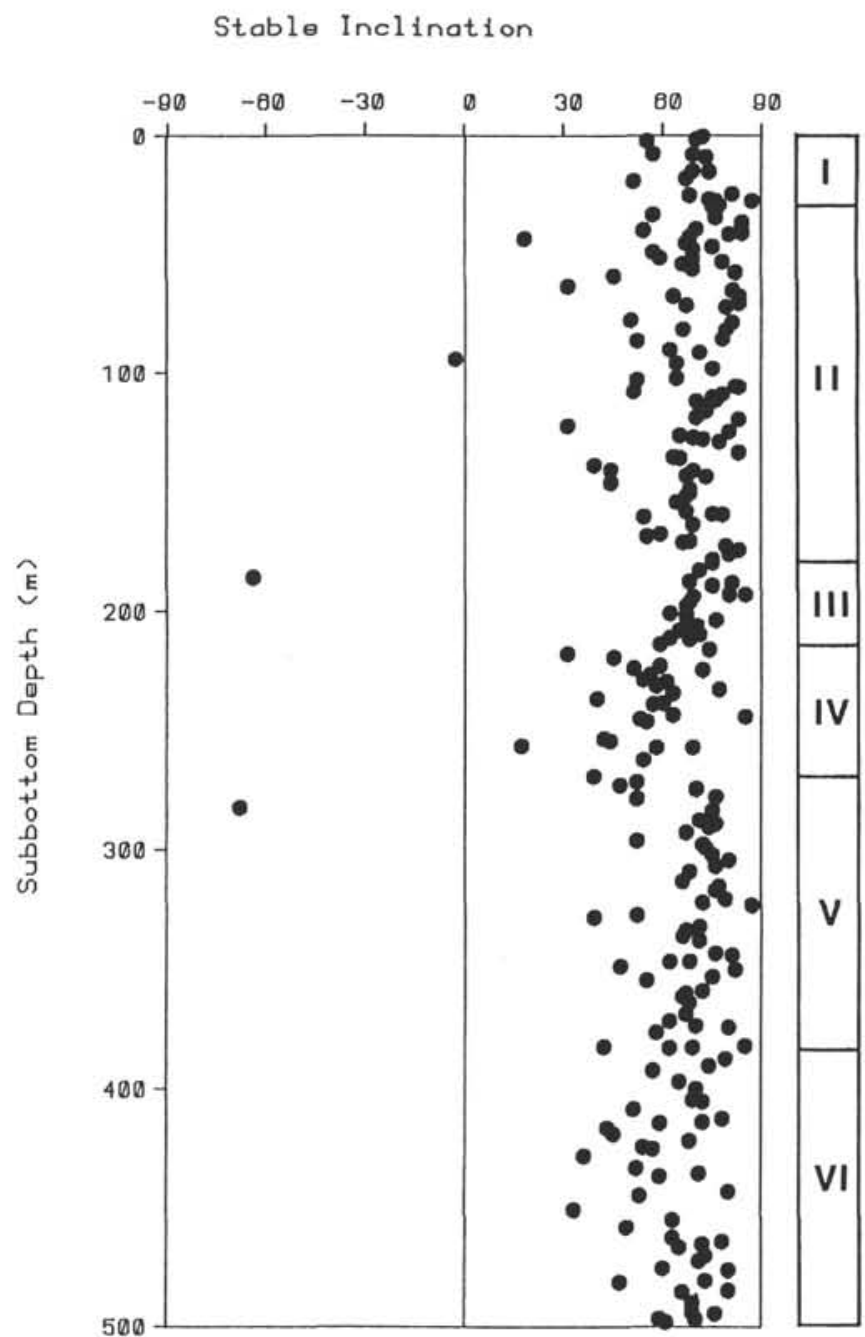

Figure 8. Plot of stable inclinations vs. depth for Hole 735B.

part of Fig. 16). Samples of high grade show values that vary from $4.30 \times 10^{-5}$ to $3.44 \times 10^{-2} \mathrm{cgs}$. The arithmetic mean value of $1.73 \times 10^{-3}$ (a solid triangle in the upper part of Fig. 16) decreases to $7.42 \times 10^{-4} \mathrm{cgs}$ (an open triangle in the upper part of Fig. 16) when excluding the highest sample.

Susceptibility values of most of the samples range between $10^{-4}$ and $10^{-3} \mathrm{cgs}$, which is similar to the overall result described in the previous section, and thus is consistent with the previously reported range. However, many of samples have values larger than $10^{-3} \mathrm{cgs}$, which makes these means higher than in the previous studies. Figure 16 shows similar distributions for the three grades, indicating no essential difference among the metamorphism/alteration grades, although close observation might reveal relatively higher frequencies at the lower range in high-grade samples.

Koenigsberger ratios calculated for samples having both NRM intensity and susceptibility measurements (described above in this section) are presented as a histogram in Figure 17. Solid triangles indicate the arithmetic means and an open triangle is a reduced mean produced by leaving out the highest three samples (Samples 118-735B-24R-4, $26 \mathrm{~cm}, 118-735 \mathrm{~B}-$ $66 \mathrm{R}-2,86 \mathrm{~cm}$, and $118-735 \mathrm{~B}-66 \mathrm{R}-3,60 \mathrm{~cm}$ ) from the mean calculation for high-grade samples.

All of the histograms for the three metamorphism/alteration grades in Figure 17 indicate that the majority of values 
Table 2. Calculated inclinations within Hole 735B.

\begin{tabular}{|c|c|c|c|c|c|c|c|}
\hline $\begin{array}{l}\text { Core/section } \\
\text { interval }(\mathrm{cm})\end{array}$ & $\begin{array}{l}\text { Depth } \\
\text { (mbsf) }\end{array}$ & $\underset{(\mathrm{kA} / \mathrm{m})}{I_{n r m}}$ & $\underset{(\mathrm{kA} / \mathrm{m})}{J_{n r m}}$ & $I_{c}$ & $I_{s}$ & $\underset{(\mathrm{kA} / \mathrm{m})}{J_{s t}}$ & $I_{c s}$ \\
\hline $\begin{array}{l}118-735 \mathrm{~B}- \\
1 \mathrm{D}-1,19\end{array}$ & 0.19 & 69 & $3.81 \mathrm{E}-04$ & -60.5 & 72 & $3.81 \mathrm{E}-04$ & -60.4 \\
\hline ID-1, 141 & 1.41 & 72 & $3.79 \mathrm{E}-04$ & -60.4 & 70 & $3.79 \mathrm{E}-04$ & -60.5 \\
\hline $1 D-2,59$ & 2.09 & 54 & $2.98 \mathrm{E}-04$ & -60.4 & 55 & $3.21 \mathrm{E}-04$ & -60.4 \\
\hline $2 \mathrm{D}-1,131$ & 7.63 & 62 & $4.38 \mathrm{E}-04$ & -60.5 & 57 & 4.38E-04 & -60.6 \\
\hline 2D-1, 139 & 7.89 & 78 & $4.18 \mathrm{E}-04$ & -60.5 & 69 & 4.18E-04 & -60.5 \\
\hline $2 \mathrm{D}-2,99$ & 8.99 & 69 & $3.34 \mathrm{E}-04$ & -60.4 & 73 & $3.34 \mathrm{E}-04$ & -60.4 \\
\hline $3 \mathrm{D}-1,42$ & 14.92 & 71 & 8.07E-04 & -60.9 & 69 & $7.89 \mathrm{E}-04$ & -60.9 \\
\hline $3 \mathrm{D}-1,58$ & 15.08 & 75 & $8.74 \mathrm{E}-06$ & -60.0 & 74 & $8.74 \mathrm{E}-06$ & -60.0 \\
\hline $3 \mathrm{D}-1,60$ & 15.10 & 76 & $5.86 \mathrm{E}-04$ & -60.7 & 74 & $5.86 \mathrm{E}-04$ & -60.7 \\
\hline $4 \mathrm{D}-1,32$ & 17.82 & 71 & 8.59E-04 & -61.0 & 67 & $7.26 \mathrm{E}-04$ & -60.9 \\
\hline $4 \mathrm{D}-2,7$ & 19.07 & 57 & $2.44 \mathrm{E}-03$ & -62.9 & 51 & $2.44 \mathrm{E}-03$ & -62.9 \\
\hline $6 \mathrm{D}-1,56$ & 24.56 & 80 & 1.67E-04 & -60.2 & 81 & $1.55 \mathrm{E}-04$ & -60.2 \\
\hline $6 \mathrm{D}-1,111$ & 25.11 & 68 & $2.05 \mathrm{E}-04$ & -60.3 & 68 & $2.05 \mathrm{E}-04$ & -60.3 \\
\hline $7 \mathrm{D}-1,74$ & 26.74 & 75 & 6.37E-04 & -60.7 & 74 & $6.37 \mathrm{E}-04$ & -60.7 \\
\hline $7 \mathrm{D}-1,134$ & 27.34 & 77 & $1.11 \mathrm{E}-03$ & -61.2 & 76 & $1.11 \mathrm{E}-03$ & -61.2 \\
\hline $7 \mathrm{D}-2,9$ & 27.39 & 86 & $6.71 \mathrm{E}-04$ & -60.7 & 87 & $6.71 \mathrm{E}-04$ & -60.7 \\
\hline $8 D-1,22$ & 29.42 & 63 & $3.89 \mathrm{E}-04$ & -60.5 & 77 & $2.18 \mathrm{E}-04$ & -60.2 \\
\hline $8 D-1,45$ & 29.65 & 75 & 5.62E-04 & -60.6 & 75 & $5.62 \mathrm{E}-04$ & -60.6 \\
\hline $9 \mathrm{D}-1,9$ & 33.29 & 61 & $4.36 \mathrm{E}-04$ & -60.5 & 57 & $4.41 \mathrm{E}-04$ & -60.6 \\
\hline $9 D-1,120$ & 34.40 & 79 & $3.11 \mathrm{E}-04$ & -60.3 & 76 & $3.11 \mathrm{E}-04$ & -60.4 \\
\hline $10 \mathrm{D}-1,24$ & 36.44 & 86 & $2.31 \mathrm{E}-04$ & -60.2 & 84 & 2.29E-04 & -60.2 \\
\hline $11 \mathrm{D}-1,3$ & 39.23 & -68 & $1.31 \mathrm{E}-04$ & 67.5 & 70 & $1.42 \mathrm{E}-03$ & -61.6 \\
\hline $11 D-1,6$ & 39.26 & -76 & $9.90 \mathrm{E}-04$ & 69.4 & - & - & - \\
\hline $12 \mathrm{R}-1,32$ & 39.72 & 54 & $4.77 \mathrm{E}-03$ & -65.6 & 54 & $4.77 \mathrm{E}-03$ & -65.6 \\
\hline $12 \mathrm{R}-1,131$ & 40.71 & 70 & $4.03 E-04$ & -60.5 & 84 & $2.00 \mathrm{E}-04$ & -60.2 \\
\hline $12 R-2,36$ & 41.26 & 37 & $6.24 \mathrm{E}-04$ & -60.8 & 80 & $5.11 \mathrm{E}-04$ & -60.6 \\
\hline $12 \mathrm{R}-3,8$ & 42.48 & 14 & $2.95 \mathrm{E}-04$ & -60.3 & 68 & 2. $19 \mathrm{E}-04$ & -60.3 \\
\hline $12 \mathrm{R}-3,83$ & 43.23 & 18 & $1.33 \mathrm{E}-03$ & -61.4 & 18 & $1.42 \mathrm{E}-03$ & -61.5 \\
\hline $13 \mathrm{R}-1,102$ & 45.02 & 67 & $1.22 \mathrm{E}-03$ & -61.4 & 67 & $1.41 \mathrm{E}-03$ & -61.6 \\
\hline $13 \mathrm{R}-2,55$ & 46.55 & 79 & $9.69 \mathrm{E}-05$ & -60.1 & 75 & $2.18 \mathrm{E}-04$ & -60.3 \\
\hline $13 \mathrm{R}-3,22$ & 47.72 & 24 & $3.42 \mathrm{E}-04$ & -60.4 & 69 & $1.95 \mathrm{E}-04$ & -60.2 \\
\hline $13 \mathrm{R}-3,141$ & 48.91 & -59 & $4.50 \mathrm{E}-04$ & -59.4 & 57 & - & - \\
\hline $14 \mathrm{R}-1,11$ & 51.31 & 58 & 2.19E-03 & -62.6 & 59 & - & - \\
\hline $14 \mathrm{R}-1,35$ & 51.55 & 66 & $2.15 \mathrm{E}-03$ & -62.4 & 69 & $2.28 \mathrm{E}-03$ & -62.6 \\
\hline $14 \mathrm{R}-2,22$ & 52.92 & 84 & $5.44 \mathrm{E}-04$ & -60.6 & 78 & $5.65 \mathrm{E}-04$ & -60.6 \\
\hline $14 \mathrm{R}-3,8$ & 53.77 & 56 & $9.11 \mathrm{E}-04$ & -61.1 & 66 & $1.07 \mathrm{E}-03$ & -61.3 \\
\hline $14 \mathrm{R}-4,22$ & 55.92 & 69 & $2.58 \mathrm{E}-03$ & -62.9 & 69 & $2.58 \mathrm{E}-03$ & -62.9 \\
\hline $15 \mathrm{R}-1,102$ & 57.22 & -70 & $9.84 \mathrm{E}-03$ & -45.2 & 82 & $3.86 \mathrm{E}-04$ & -60.4 \\
\hline $15 \mathrm{R}-2,128$ & 58.98 & -70 & $4.12 \mathrm{E}-03$ & -54.6 & 45 & $1.82 \mathrm{E}-04$ & -60.2 \\
\hline $16 \mathrm{R}-1,65$ & 62.45 & -64 & $4.64 \mathrm{E}-02$ & 31.5 & - & - & - \\
\hline $16 \mathrm{R}-1,139$ & 63.19 & -19 & $9.11 \mathrm{E}-05$ & -59.9 & 31 & $1.21 \mathrm{E}-04$ & -60.2 \\
\hline $16 \mathrm{R}-3,9$ & 64.78 & 75 & 2.37E-04 & -60.3 & 81 & 2.57E-04 & -60.3 \\
\hline $16 R-4,77$ & 67.07 & 87 & $2.70 \mathrm{E}-04$ & -60.3 & 83 & $2.90 \mathrm{E}-04$ & -60.3 \\
\hline $16 R-5,24$ & 67.30 & -75 & $9.37 \mathrm{E}-04$ & -58.9 & 63 & $7.00 \mathrm{E}-05$ & -60.1 \\
\hline $18 \mathrm{R}-2,12$ & 70.12 & 85 & $2.20 \mathrm{E}-03$ & -62.1 & 83 & $2.04 \mathrm{E}-03$ & -62.0 \\
\hline $18 \mathrm{R}-2,110$ & 71.10 & 61 & $1.90 \mathrm{E}-05$ & -60.0 & 67 & $1.98 \mathrm{E}-05$ & -60.0 \\
\hline $18 \mathrm{R}-3,29$ & 71.79 & 81 & $2.33 \mathrm{E}-05$ & -60.0 & 79 & 2.12E-05 & -60.0 \\
\hline $19 \mathrm{R}-2,128$ & 77.48 & 43 & $1.06 \mathrm{E}-03$ & -61.3 & 50 & $1.13 \mathrm{E}-03$ & -61.4 \\
\hline $19 \mathrm{R}-3,73$ & 78.43 & 90 & $1.20 \mathrm{E}-03$ & -61.1 & 81 & $1.73 \mathrm{E}-03$ & -61.8 \\
\hline 19R-5, 109 & 81.37 & 77 & $1.27 \mathrm{E}-03$ & -61.4 & 66 & - & - \\
\hline $19 \mathrm{R}-5,126$ & 81.54 & -59 & 3.23E-04 & -59.6 & 79 & $2.64 \mathrm{E}-04$ & -60.3 \\
\hline $20 \mathrm{R}-1,120$ & 85.40 & 73 & $4.26 \mathrm{E}-03$ & -64.4 & 78 & $3.16 \mathrm{E}-03$ & -63.2 \\
\hline $20 \mathrm{R}-2,54$ & 86.24 & 73 & $1.55 \mathrm{E}-03$ & -61.7 & 52 & $1.61 \mathrm{E}-03$ & -62.0 \\
\hline $21 \mathrm{R}-1,91$ & 90.11 & 61 & $2.71 \mathrm{E}-03$ & -63.1 & 62 & $2.39 \mathrm{E}-03$ & -62.8 \\
\hline $21 R-2,49$ & 91.19 & 60 & $1.48 \mathrm{E}-03$ & -61.8 & 71 & $1.55 \mathrm{E}-03$ & -61.8 \\
\hline $22 \mathrm{R}-1,2$ & 94.02 & 24 & $3.25 \mathrm{E}-04$ & -60.4 & -3 & 2.17E-04 & -59.8 \\
\hline $22 \mathrm{R}-2,41$ & 95.91 & 69 & $1.39 \mathrm{E}-04$ & -60.2 & 64 & $1.37 \mathrm{E}-04$ & -60.2 \\
\hline $22 \mathrm{R}-3,18$ & 98.18 & 80 & $1.37 \mathrm{E}-03$ & -61.5 & 75 & $1.35 \mathrm{E}-03$ & -61.6 \\
\hline $23 \mathrm{R}-2,34$ & 102.34 & 74 & $6.86 \mathrm{E}-04$ & -60.8 & 64 & $7.13 \mathrm{E}-04$ & -60.9 \\
\hline $23 \mathrm{R}-3,43$ & 102.98 & -30 & $1.22 \mathrm{E}-02$ & -44.6 & 52 & $3.56 \mathrm{E}-03$ & -64.2 \\
\hline $23 \mathrm{R}-4,120$ & 105.20 & 54 & $4.92 \mathrm{E}-06$ & -60.0 & - & - & - \\
\hline $23 \mathrm{R}-5,13$ & 105.63 & 75 & $2.04 \mathrm{E}-04$ & -60.2 & 82 & $1.94 \mathrm{E}-04$ & -60.2 \\
\hline $24 \mathrm{R}-1,47$ & 105.97 & 79 & $1.94 \mathrm{E}-02$ & -73.7 & 83 & $9.71 \mathrm{E}-03$ & -67.9 \\
\hline $24 \mathrm{R}-2,95$ & 107.95 & 58 & $3.76 \mathrm{E}-02$ & -85.2 & 51 & $2.54 \mathrm{E}-02$ & -81.2 \\
\hline $24 \mathrm{R}-3,50$ & 109.00 & 76 & $1.09 \mathrm{E}-02$ & -70.0 & 78 & $1.10 \mathrm{E}-02$ & -69.4 \\
\hline $24 \mathrm{R}-4,26$ & 110.23 & 80 & $6.80 \mathrm{E}-03$ & -66.3 & 75 & $6.75 \mathrm{E}-03$ & -66.6 \\
\hline $25 \mathrm{R}-1,112$ & 111.62 & 71 & $1.33 \mathrm{E}-03$ & -61.5 & 76 & $1.25 \mathrm{E}-03$ & -61.4 \\
\hline $25 \mathrm{R}-2,5$ & 112.05 & 63 & $1.80 \mathrm{E}-04$ & -60.2 & 70 & $1.75 \mathrm{E}-04$ & -60.2 \\
\hline $25 \mathrm{R}-3,137$ & 114.87 & 73 & $6.78 \mathrm{E}-04$ & -60.8 & 73 & $7.10 \mathrm{E}-04$ & -60.8 \\
\hline $26 \mathrm{R}-1,62$ & 116.22 & 79 & $3.00 \mathrm{E}-03$ & -63.0 & 73 & $2.98 \mathrm{E}-03$ & -63.3 \\
\hline $26 \mathrm{R}-3,36$ & 118.86 & 71 & $5.87 \mathrm{E}-04$ & -60.7 & - & - & - \\
\hline $26 \mathrm{R}-3,112$ & 119.62 & 78 & $2.08 \mathrm{E}-03$ & -62.2 & 78 & $1.83 \mathrm{E}-03$ & -61.8 \\
\hline $27 \mathrm{R}-1,84$ & 122.34 & 30 & $1.27 \mathrm{E}-03$ & -61.6 & 31 & $1.25 \mathrm{E}-03$ & -61.6 \\
\hline $27 \mathrm{R}-3,32$ & 124.82 & 75 & $6.02 \mathrm{E}-04$ & -60.7 & 80 & $5.95 \mathrm{E}-04$ & -60.6 \\
\hline $27 \mathrm{R}-4,52$ & 126.52 & 46 & $2.90 \mathrm{E}-04$ & -60.4 & 65 & $1.96 \mathrm{E}-04$ & -60.2 \\
\hline $28 \mathrm{R}-1,83$ & 127.33 & 70 & $2.77 \mathrm{E}-04$ & -60.3 & 69 & 2.77E- 04 & -60.3 \\
\hline
\end{tabular}


Table 2 (continued).

\begin{tabular}{|c|c|c|c|c|c|c|c|}
\hline $\begin{array}{l}\text { Core/section } \\
\text { interval }(\mathrm{cm})\end{array}$ & $\begin{array}{l}\text { Depth } \\
\text { (mbsf) }\end{array}$ & $\underset{(\mathrm{kA} / \mathrm{m})}{I_{n r m}}$ & $\underset{(\mathrm{kA} / \mathrm{m})}{J_{n r m}}$ & $I_{c}$ & $I_{s}$ & $\begin{array}{c}J_{s t} \\
(\mathrm{kA} / \mathrm{m})\end{array}$ & $I_{c s}$ \\
\hline 28R-1, 99 & 127.49 & - & $2.45 \mathrm{E}-02$ & - & - & - & - \\
\hline 28R-2, 12 & 127.96 & 68 & $7.24 \mathrm{E}-03$ & -67.4 & 72 & $7.15 \mathrm{E}-03$ & -67.1 \\
\hline $28 \mathrm{R}-2,114$ & 128.98 & 84 & $9.29 \mathrm{E}-04$ & -60.9 & 77 & $9.25 \mathrm{E}-04$ & -61.0 \\
\hline $29 \mathrm{R}-1,119$ & 132.69 & -80 & $6.54 \mathrm{E}-03$ & -51.5 & - & - & - \\
\hline $29 \mathrm{R}-2,46$ & 133.46 & 52 & $5.76 \mathrm{E}-04$ & -60.7 & 83 & $1.18 \mathrm{E}-03$ & -61.2 \\
\hline $29 \mathrm{R}-3,113$ & 135.44 & 39 & 8.97E-04 & -61.1 & 63 & 2.22E-03 & -62.6 \\
\hline $29 \mathrm{R}-4,19$ & 135.85 & 60 & $1.81 \mathrm{E}-04$ & -60.2 & 65 & 2.78E-04 & -60.3 \\
\hline $30 \mathrm{R}-2,97$ & 138.91 & -44 & $3.40 \mathrm{E}-02$ & -5.4 & 39 & 4.07E-04 & -60.5 \\
\hline $30 \mathrm{R}-3,137$ & 140.87 & -55 & $2.80 \mathrm{E}-02$ & -6.6 & 44 & 4.30E-04 & -60.5 \\
\hline $30 \mathrm{R}-4,14$ & 141.14 & -64 & $1.96 \mathrm{E}-03$ & -57.4 & 69 & $4.80 \mathrm{E}-04$ & -60.6 \\
\hline $30 \mathrm{R}-5,91$ & 143.21 & -72 & $2.37 \mathrm{E}-03$ & -57.0 & 67 & 4.70E-04 & -60.6 \\
\hline $31 \mathrm{R}-1,16$ & 143.66 & -67 & 2.79E-03 & -56.4 & 73 & $1.09 \mathrm{E}-03$ & -61.3 \\
\hline $31 \mathrm{R}-2,120$ & 146.20 & -75 & $1.13 \mathrm{E}-03$ & -58.7 & 44 & $7.21 \mathrm{E}-04$ & -60.9 \\
\hline $31 \mathrm{R}-4,118$ & 148.98 & -76 & $1.74 \mathrm{E}-03$ & -58.0 & 68 & $4.91 \mathrm{E}-04$ & -60.6 \\
\hline $32 \mathrm{R}-1,64$ & 149.14 & -71 & $1.00 \mathrm{E}-01$ & 65.3 & - & - & - \\
\hline $32 \mathrm{R}-2,60$ & 150.60 & 65 & $3.94 \mathrm{E}-03$ & -64.4 & 68 & 4.33E-03 & -64.7 \\
\hline $32 \mathrm{R}-3,14$ & 151.64 & 66 & $9.73 \mathrm{E}-04$ & -61.2 & 67 & $1.10 \mathrm{E}-03$ & -61.3 \\
\hline $33 \mathrm{R}-1,81$ & 154.31 & -63 & $1.13 \mathrm{E}-03$ & -58.6 & 64 & $1.28 \mathrm{E}-03$ & -61.5 \\
\hline $33 \mathrm{R}-4,27$ & 158.27 & -78 & $3.25 \mathrm{E}-03$ & -55.9 & 67 & $3.26 \mathrm{E}-03$ & -63.7 \\
\hline $33 \mathrm{R}-4,129$ & 159.29 & -69 & $5.52 \mathrm{E}-04$ & -59.3 & 75 & 8.00E-04 & -60.9 \\
\hline $34 \mathrm{R}-1,103$ & 159.53 & 5 & $5.65 \mathrm{E}-04$ & -60.5 & 78 & $1.10 \mathrm{E}-03$ & -61.2 \\
\hline $34 \mathrm{R}-2,56$ & 160.36 & -83 & $1.59 \mathrm{E}-03$ & -58.2 & 54 & $6.90 \mathrm{E}-04$ & -60.9 \\
\hline $34 \mathrm{R}-4,30$ & 162.76 & 75 & 4.44E-05 & -59.9 & 78 & $2.10 \mathrm{E}-04$ & -60.2 \\
\hline $35 \mathrm{R}-1,29$ & 163.79 & 71 & $6.01 \mathrm{E}-04$ & -60.4 & 69 & $9.95 \mathrm{E}-04$ & -61.2 \\
\hline $35 \mathrm{R}-3,133$ & 167.65 & 44 & $7.52 \mathrm{E}-04$ & -60.9 & - & - & - \\
\hline $35 \mathrm{R}-4,65$ & 168.47 & 40 & $2.04 \mathrm{E}-03$ & -62.5 & 55 & $2.41 \mathrm{E}-03$ & -62.9 \\
\hline $35 \mathrm{R}-5,131$ & 170.81 & -71 & $2.45 \mathrm{E}-04$ & -59.7 & 68 & $1.94 \mathrm{E}-04$ & -60.2 \\
\hline $36 \mathrm{R}-1,14$ & 171.14 & -54 & $9.05 \mathrm{E}-02$ & 48.8 & 66 & 2.98E-04 & -60.4 \\
\hline $36 \mathrm{R}-2,11$ & 172.61 & -74 & $9.22 \mathrm{E}-04$ & -58.9 & 79 & $8.74 \mathrm{E}-04$ & -60.9 \\
\hline $36 \mathrm{R}-3,36$ & 174.36 & 75 & 2.55E-03 & -62.8 & 83 & $4.61 \mathrm{E}-03$ & -64.3 \\
\hline $37 \mathrm{R}-1,11$ & 176.11 & -31 & 1.03E-03 & -58.8 & 80 & $1.85 \mathrm{E}-03$ & -62.0 \\
\hline $37 \mathrm{R}-2,90$ & 178.40 & 70 & $3.23 \mathrm{E}-03$ & -63.5 & 75 & $3.48 \mathrm{E}-03$ & -63.6 \\
\hline $37 \mathrm{R}-3,80$ & 179.80 & -67 & $5.54 \mathrm{E}-03$ & -52.4 & 75 & $1.29 \mathrm{E}-03$ & -61.4 \\
\hline $38 \mathrm{R}-1,81$ & 181.81 & -62 & $5.04 \mathrm{E}-02$ & 34.2 & - & - & - \\
\hline $38 \mathrm{R}-2,15$ & 182.65 & -61 & $2.60 \mathrm{E}-06$ & -60.0 & - & - & - \\
\hline $38 \mathrm{R}-2,33$ & 182.83 & -62 & $5.87 \mathrm{E}-03$ & -51.9 & 71 & $2.82 \mathrm{E}-04$ & -60.3 \\
\hline $38 \mathrm{R}-4,28$ & 185.78 & 48 & $1.53 \mathrm{E}-03$ & -58.0 & -64 & $2.12 \mathrm{E}-03$ & -62.5 \\
\hline $39 \mathrm{R}-1,145$ & 187.45 & -49 & $1.21 \mathrm{E}-03$ & -58.4 & 68 & $1.38 \mathrm{E}-03$ & -61.6 \\
\hline 39R-2, 68 & 188.18 & 79 & 1.29E-03 & -61.4 & 81 & $1.34 \mathrm{E}-03$ & -61.4 \\
\hline $39 \mathrm{R}-3,21$ & 189.21 & 67 & 2.21E-03 & -62.5 & 75 & $2.45 \mathrm{E}-03$ & -62.7 \\
\hline $40 \mathrm{R}-2,62$ & 193.12 & 84 & $1.14 \mathrm{E}-03$ & -61.1 & 80 & $1.53 \mathrm{E}-03$ & -61.6 \\
\hline $40 \mathrm{R}-3,14$ & 193.03 & -78 & 3.61E-03 & -55.6 & 85 & $1.06 \mathrm{E}-03$ & -61.0 \\
\hline $40 \mathrm{R}-3,96$ & 193.85 & 57 & $1.54 \mathrm{E}-03$ & -60.2 & - & - & $\overline{-}$ \\
\hline $40 \mathrm{R}-5,13$ & 196.05 & 16 & $1.04 \mathrm{E}-03$ & -61.1 & 68 & $1.92 \mathrm{E}-03$ & -62.2 \\
\hline $41 \mathrm{R}-2,30$ & 197.72 & 3 & $5.85 \mathrm{E}-03$ & -65.3 & 67 & $5.29 \mathrm{E}-03$ & -65.6 \\
\hline $41 \mathrm{R}-4,68$ & 200.86 & 65 & $1.08 \mathrm{E}-03$ & -61.3 & 62 & 2.65E-03 & -63.8 \\
\hline $42 \mathrm{R}-1,94$ & 201.94 & 58 & $2.15 \mathrm{E}-03$ & -62.6 & 67 & $2.42 \mathrm{E}-03$ & -62.7 \\
\hline $42 \mathrm{R}-2,119$ & 203.69 & -50 & $2.57 \mathrm{E}-03$ & -56.5 & 76 & $1.10 \mathrm{E}-03$ & -61.2 \\
\hline $42 R-4,62$ & 206.12 & -40 & $2.68 \mathrm{E}-03$ & -56.5 & 70 & 2.04E-03 & -62.3 \\
\hline $43 \mathrm{R}-1,126$ & 207.26 & 32 & $1.20 \mathrm{E}-03$ & -59.8 & 69 & $2.41 \mathrm{E}-03$ & -62.7 \\
\hline $43 \mathrm{R}-2,52$ & 207.96 & 3 & $4.25 \mathrm{E}-03$ & -63.8 & 65 & $3.75 \mathrm{E}-03$ & -64.2 \\
\hline $43 R-4,17$ & 209.75 & -47 & $1.53 \mathrm{E}-03$ & -58.0 & - & - & - \\
\hline $43 R-4,64$ & 211.14 & -75 & $7.42 \mathrm{E}-03$ & -49.6 & 62 & $1.60 \mathrm{E}-03$ & -61.9 \\
\hline 44R-1, 68 & 211.68 & 62 & $5.85 \mathrm{E}-04$ & -60.7 & 68 & $8.02 \mathrm{E}-04$ & -60.9 \\
\hline $44 \mathrm{R}-2,6$ & 212.56 & -81 & $1.00 \mathrm{E}-01$ & 72.5 & - & - & - \\
\hline $44 \mathrm{R}-2,131$ & 213.81 & -78 & 4.24E-03 & -54.7 & 59 & $8.91 \mathrm{E}-04$ & -61.1 \\
\hline $45 \mathrm{R}-1,1$ & 216.01 & -75 & $2.10 \mathrm{E}-03$ & -57.5 & - & - & - \\
\hline $45 \mathrm{R}-2,15$ & 217.65 & -66 & $1.18 \mathrm{E}-04$ & -59.9 & 31 & $5.00 \mathrm{E}-05$ & -60.1 \\
\hline $45 \mathrm{R}-3,74$ & 219.26 & -67 & $5.90 \mathrm{E}-04$ & -59.3 & 45 & $3.60 \mathrm{E}-05$ & -60.0 \\
\hline $46 \mathrm{R}-2,21$ & 222.71 & -2 & $6.45 \mathrm{E}-04$ & -59.4 & 59 & $1.25 \mathrm{E}-03$ & -61.6 \\
\hline $46 \mathrm{R}-2,128$ & 223.78 & -71 & $5.99 \mathrm{E}-04$ & -59.3 & 51 & $1.89 \mathrm{E}-04$ & -60.2 \\
\hline $46 \mathrm{R}-3,58$ & 224.51 & -66 & $1.58 \mathrm{E}-02$ & -31.5 & 72 & $1.34 \mathrm{E}-03$ & -61.5 \\
\hline $47 \mathrm{R}-1,54$ & 226.54 & -83 & $6.80 \mathrm{E}-02$ & 65.0 & - & - & - \\
\hline $47 R-2,111$ & 228.56 & -75 & $3.12 \mathrm{E}-02$ & 12.9 & 54 & $1.18 \mathrm{E}-03$ & -61.5 \\
\hline $47 \mathrm{R}-3,50$ & 229.50 & -76 & $3.81 \mathrm{E}-03$ & -55.2 & 61 & $1.56 \mathrm{E}-04$ & -60.2 \\
\hline $47 \mathrm{R}-4,64$ & 231.01 & 45 & $5.77 \mathrm{E}-04$ & -60.7 & - & - & - \\
\hline $48 \mathrm{R}-2,24$ & 232.58 & -75 & $3.32 \mathrm{E}-02$ & 16.9 & - & - & - \\
\hline $48 \mathrm{R}-3,53$ & 234.20 & -67 & $2.81 \mathrm{E}-02$ & 0.4 & 63 & $9.42 \mathrm{E}-04$ & -61.1 \\
\hline $48 \mathrm{R}-4,82$ & 236.32 & -73 & $3.46 \mathrm{E}-02$ & 21.5 & 40 & $7.60 \mathrm{E}-04$ & -61.0 \\
\hline $49 \mathrm{R}-1,36$ & 236.36 & -46 & $2.68 \mathrm{E}-02$ & -14.3 & - & - & - \\
\hline $49 \mathrm{R}-2,89$ & 238.39 & -61 & $1.31 \mathrm{E}-02$ & -38.5 & 60 & $1.98 \mathrm{E}-03$ & -62.4 \\
\hline 50R-1, 77 & 238.77 & -25 & $2.54 \mathrm{E}-02$ & -30.1 & 57 & $1.42 \mathrm{E}-03$ & -61.7 \\
\hline $50 \mathrm{R}-2,133$ & 240.42 & 67 & $4.56 \mathrm{E}-02$ & -84.8 & - & - & - \\
\hline $50 \mathrm{R}-4,87$ & 243.37 & -58 & $8.88 \mathrm{E}-03$ & -46.5 & 63 & $1.41 \mathrm{E}-04$ & -60.2 \\
\hline $51 R-1,102$ & 244.02 & -73 & $2.46 \mathrm{E}-02$ & -5.5 & 85 & $1.34 \mathrm{E}-04$ & -60.1 \\
\hline $51 \mathrm{R}-2,60$ & 244.83 & -43 & $1.75 \mathrm{E}-02$ & -32.0 & 53 & $5.41 \mathrm{E}-03$ & -66.2 \\
\hline 51R-3, 58 & 246.08 & 71 & $1.48 \mathrm{E}-02$ & -72.4 & - & - & - \\
\hline
\end{tabular}


Table 2 (continued).

\begin{tabular}{|c|c|c|c|c|c|c|c|}
\hline $\begin{array}{l}\text { Core/section } \\
\text { interval }(\mathrm{cm})\end{array}$ & $\begin{array}{l}\text { Depth } \\
\text { (mbsf) }\end{array}$ & $\underset{(\mathrm{kA} / \mathrm{m})}{I_{n r m}}$ & $\underset{(\mathrm{kA} / \mathrm{m})}{J_{n r m}}$ & $I_{c}$ & $I_{s}$ & $\underset{(\mathrm{kA} / \mathrm{m})}{J_{s t}}$ & $I_{c s}$ \\
\hline $52 \mathrm{R}-1,115$ & 249.15 & -49 & $5.66 \mathrm{E}-02$ & 26.1 & - & - & - \\
\hline $52 R-4,69$ & 253.19 & -63 & $3.57 \mathrm{E}-02$ & 15.6 & 42 & $4.64 \mathrm{E}-04$ & -60.6 \\
\hline $53 \mathrm{R}-1,128$ & 254.28 & -55 & $1.16 \mathrm{E}-02$ & -40.9 & 44 & $5.05 \mathrm{E}-04$ & -60.6 \\
\hline $53 R-2,94$ & 255.34 & -74 & $1.76 \mathrm{E}-02$ & -27.8 & - & - & - \\
\hline $53 \mathrm{R}-3,15$ & 256.15 & -60 & $3.06 \mathrm{E}-02$ & 3.1 & 17 & $4.50 \mathrm{E}-04$ & -60.5 \\
\hline $53 \mathrm{R}-3,95$ & 256.95 & -85 & $1.20 \mathrm{E}-02$ & -42.3 & 58 & - & - \\
\hline $53 \mathrm{R}-4,18$ & 256.98 & -77 & $8.95 \mathrm{E}-03$ & -46.9 & 69 & $1.42 \mathrm{E}-04$ & -60.2 \\
\hline $54 \mathrm{R}-3,125$ & 262.25 & -75 & $3.40 \mathrm{E}-02$ & 20.1 & 53 & $2.24 \mathrm{E}-04$ & -60.3 \\
\hline 54R-5, 117 & 265.17 & -58 & $2.10 \mathrm{E}-02$ & -21.1 & 36 & $5.53 \mathrm{E}-03$ & -66.5 \\
\hline 55R-I, 107 & 266.07 & -57 & $9.08 \mathrm{E}-02$ & 52.1 & - & - & - \\
\hline $55 \mathrm{R}-3,130$ & 269.30 & -78 & $5.67 \mathrm{E}-02$ & 55.0 & 39 & $3.71 \mathrm{E}-04$ & -60.5 \\
\hline $56 \mathrm{R}-2,11$ & 271.61 & -67 & $8.95 \mathrm{E}-03$ & -46.4 & 52 & 2.55E-04 & -60.3 \\
\hline $56 \mathrm{R}-2,144$ & 272.94 & 2 & $4.14 \mathrm{E}-05$ & -60.0 & 47 & $6.40 \mathrm{E}-05$ & -60.1 \\
\hline $56 \mathrm{R}-4,11$ & 274.38 & 17 & $5.73 \mathrm{E}-04$ & -60.6 & 70 & $4.51 \mathrm{E}-04$ & -60.5 \\
\hline $56 R-4,58$ & 274.81 & 61 & $6.11 \mathrm{E}-04$ & -60.7 & 70 & - & - \\
\hline $57 \mathrm{R}-2,135$ & 277.85 & 5 & $1.15 \mathrm{E}-03$ & -61.3 & 76 & $2.98 \mathrm{E}-03$ & -63.2 \\
\hline $57 \mathrm{R}-3,71$ & 278.64 & 17 & $4.41 \mathrm{E}-03$ & -64.7 & 52 & $5.80 \mathrm{E}-03$ & -66.7 \\
\hline $58 \mathrm{R}-2,33$ & 282.33 & -59 & $2.75 \mathrm{E}-03$ & - & -68 & $4.43 \mathrm{E}-03$ & - \\
\hline $58 \mathrm{R}-3,34$ & 283.59 & 61 & $2.73 \mathrm{E}-03$ & -63.2 & 75 & 2.67E-03 & -62.8 \\
\hline 59R-2, 96 & 287.74 & 24 & $1.83 \mathrm{E}-03$ & -62.1 & 71 & $2.30 \mathrm{E}-03$ & -62.5 \\
\hline $59 \mathrm{R}-3,70$ & 289.20 & 63 & $2.23 \mathrm{E}-03$ & -62.6 & 76 & $3.10 \mathrm{E}-03$ & -63.3 \\
\hline $60 \mathrm{R}-1,18$ & 290.68 & 81 & $1.86 \mathrm{E}-03$ & -62.0 & 74 & $2.73 \mathrm{E}-03$ & -62.9 \\
\hline $60 \mathrm{R}-2,120$ & 292.84 & 16 & $3.83 \mathrm{E}-03$ & -64.0 & 67 & $3.98 \mathrm{E}-03$ & -64.3 \\
\hline $61 \mathrm{R}-1,81$ & 296.31 & 26 & $9.93 \mathrm{E}-04$ & -61.2 & 52 & $1.75 \mathrm{E}-03$ & -62.2 \\
\hline $61 \mathrm{R}-2,88$ & 297.86 & -16 & $2.59 \mathrm{E}-03$ & -57.3 & - & - & - \\
\hline $61 R-3,90$ & 299.33 & 65 & $4.48 \mathrm{E}-03$ & -64.9 & 73 & $6.64 \mathrm{E}-03$ & -66.5 \\
\hline $62 \mathrm{R}-2,45$ & 302.19 & 10 & $1.74 \mathrm{E}-03$ & -61.7 & 75 & $2.68 \mathrm{E}-03$ & -62.8 \\
\hline $62 \mathrm{R}-3,104$ & 304.54 & 72 & $5.27 \mathrm{E}-03$ & -65.5 & 80 & $6.00 \mathrm{E}-03$ & -65.6 \\
\hline $63 \mathrm{R}-2,23$ & 306.96 & 79 & $4.73 \mathrm{E}-03$ & -64.6 & 76 & $5.05 \mathrm{E}-03$ & -65.0 \\
\hline $63 \mathrm{R}-3,80$ & 309.30 & 71 & $1.55 \mathrm{E}-03$ & -61.7 & 68 & $1.98 \mathrm{E}-03$ & -62.3 \\
\hline $63 R-6,28$ & 313.28 & -52 & $5.17 \mathrm{E}-04$ & -59.3 & 66 & $8.65 \mathrm{E}-04$ & -61.0 \\
\hline $64 \mathrm{R}-1,33$ & 315.33 & 79 & $3.11 \mathrm{E}-03$ & -63.1 & 77 & $3.28 \mathrm{E}-03$ & -63.3 \\
\hline $64 \mathrm{R}-2,54$ & 317.04 & -38 & $9.10 \mathrm{E}-04$ & -58.8 & 76 & $1.09 \mathrm{E}-03$ & -61.2 \\
\hline $65 \mathrm{R}-1,70$ & 320.70 & 71 & $1.98 \mathrm{E}-03$ & -62.2 & 79 & - & - \\
\hline $65 R-2,67$ & 322.17 & 63 & $3.14 \mathrm{E}-03$ & -63.6 & 72 & $3.30 \mathrm{E}-03$ & -63.6 \\
\hline $65 \mathrm{R}-3,61$ & 323.21 & 26 & $1.04 \mathrm{E}-03$ & -61.2 & 87 & $1.34 \mathrm{E}-03$ & -61.3 \\
\hline $66 \mathrm{R}-2,86$ & 327.36 & 57 & $6.78 \mathrm{E}-03$ & -67.6 & 52 & $6.65 \mathrm{E}-03$ & -67.9 \\
\hline $66 \mathrm{R}-3,60$ & 328.35 & 38 & $3.04 \mathrm{E}-03$ & -63.6 & 39 & $3.11 \mathrm{E}-03$ & -63.7 \\
\hline $66 \mathrm{R}-3,134$ & 329.34 & -63 & $2.53 \mathrm{E}-04$ & -59.7 & - & - & - \\
\hline $67 R-2,86$ & 332.26 & 62 & $1.63 \mathrm{E}-03$ & -61.9 & 71 & - & - \\
\hline $67 R-3,90$ & 333.72 & 64 & $2.26 \mathrm{E}-03$ & -62.7 & 67 & $2.42 \mathrm{E}-03$ & -62.8 \\
\hline $68 \mathrm{R}-1,119$ & 336.19 & 16 & $9.52 \mathrm{E}-04$ & -61.0 & 66 & $1.95 \mathrm{E}-03$ & -62.3 \\
\hline $68 \mathrm{R}-3,15$ & 338.15 & -56 & $8.31 \mathrm{E}-04$ & -59.7 & 71 & $6.99 \mathrm{E}-04$ & -60.8 \\
\hline $69 \mathrm{R}-2,120$ & 343.48 & 68 & $9.44 \mathrm{E}-04$ & -61.1 & 76 & $1.29 \mathrm{E}-03$ & -61.4 \\
\hline $69 \mathrm{R}-3,71$ & 344.31 & 57 & $5.62 \mathrm{E}-04$ & -60.7 & 81 & - & - \\
\hline $69 R-4,138$ & 346.88 & 22 & $5.56 \mathrm{E}-04$ & -60.6 & 68 & $7.98 \mathrm{E}-04$ & -60.9 \\
\hline $70 R-1,105$ & 347.05 & 54 & $2.15 \mathrm{E}-04$ & -60.3 & 62 & $2.20 \mathrm{E}-04$ & -60.3 \\
\hline $70 \mathrm{R}-3,4$ & 349.04 & 17 & $1.34 \mathrm{E}-03$ & -61.4 & - & - & \\
\hline $70 \mathrm{R}-4,33$ & 350.39 & 81 & $1.50 \mathrm{E}-03$ & -61.5 & 82 & $1.70 \mathrm{E}-03$ & -61.7 \\
\hline $71 \mathrm{R}-2,82$ & 353.32 & -28 & $1.48 \mathrm{E}-03$ & -59.8 & 75 & $8.10 \mathrm{E}-04$ & -60.9 \\
\hline $71 R-3,98$ & 354.83 & -2 & $1.75 \mathrm{E}-03$ & -58.5 & 55 & $1.15 \mathrm{E}-03$ & -61.4 \\
\hline $72 \mathrm{R}-3,36$ & 359.36 & 78 & $1.74 \mathrm{E}-04$ & -60.2 & 72 & 2.37E-04 & -60.3 \\
\hline $72 R-4,39$ & 360.39 & -74 & $1.96 \mathrm{E}-02$ & -22.4 & 67 & $3.43 \mathrm{E}-04$ & -60.4 \\
\hline $72 R-5,34$ & 361.69 & -38 & $1.71 \mathrm{E}-04$ & -59.8 & 66 & - & \\
\hline 72R-6, 106 & 364.56 & -44 & $2.98 \mathrm{E}-04$ & -59.6 & 68 & $5.75 \mathrm{E}-04$ & -60.7 \\
\hline $73 R-3,73$ & 369.23 & 67 & $1.91 \mathrm{E}-04$ & -60.2 & 67 & $2.54 \mathrm{E}-04$ & -60.3 \\
\hline $73 R-5,106$ & 372.09 & -62 & $1.45 \mathrm{E}-03$ & -58.1 & 62 & $2.11 \mathrm{E}-04$ & -60.3 \\
\hline $73 \mathrm{R}-7,31$ & 374.08 & 31 & $5.80 \mathrm{E}-04$ & -60.7 & 70 & 8.01E-04 & -60.9 \\
\hline $73 \mathrm{R}-7,72$ & 374.79 & 79 & $2.87 \mathrm{E}-04$ & -60.3 & - & - & - \\
\hline $74 \mathrm{R}-2,38$ & 376.88 & 47 & $5.37 \mathrm{E}-04$ & -60.7 & 58 & 8.30E-04 & -61.0 \\
\hline $74 \mathrm{R}-5,37$ & 383.26 & 30 & $7.08 \mathrm{E}-04$ & -60.8 & 69 & $1.19 \mathrm{E}-03$ & -61.4 \\
\hline $74 \mathrm{R}-6,16$ & 382.47 & -76 & $1.03 \mathrm{E}-03$ & -44.9 & 85 & $3.00 \mathrm{E}-05$ & -60.0 \\
\hline $74 \mathrm{R}-6,41$ & 382.91 & -71 & $9.01 \mathrm{E}-03$ & -46.5 & 42 & $1.20 \mathrm{E}-04$ & -60.2 \\
\hline 74R-6, 101 & 383.32 & -6 & $3.91 \mathrm{E}-04$ & -59.6 & 62 & - & \\
\hline $75 \mathrm{R}-3,48$ & 387.98 & -16 & $2.60 \mathrm{E}-04$ & -59.7 & 79 & $2.89 \mathrm{E}-04$ & -60.3 \\
\hline $75 \mathrm{R}-4,117$ & 389.93 & 74 & $2.34 \mathrm{E}-04$ & -60.3 & 74 & - & - \\
\hline $75 R-5,84$ & 390.90 & 73 & $1.44 \mathrm{E}-03$ & -61.6 & 74 & $1.59 \mathrm{E}-03$ & -61.7 \\
\hline $75 \mathrm{R}-6,75$ & 392.75 & -79 & $1.26 \mathrm{E}-04$ & -59.9 & 57 & $3.00 \mathrm{E}-05$ & -60.0 \\
\hline $76 \mathrm{R}-3,50$ & 397.50 & -72 & $9.14 \mathrm{E}-03$ & -46.4 & 65 & $9.54 \mathrm{E}-03$ & -61.1 \\
\hline $76 R-5,91$ & 400.33 & -45 & $6.36 \mathrm{E}-04$ & -59.2 & 70 & $4.42 \mathrm{E}-04$ & -60.5 \\
\hline $77 \mathrm{R}-1,135$ & 404.85 & -42 & $9.96 \mathrm{E}-04$ & -58.7 & 69 & $7.32 \mathrm{E}-04$ & -60.8 \\
\hline $77 R-2,63$ & 405.63 & -35 & $4.82 \mathrm{E}-04$ & -59.4 & 72 & - & - \\
\hline $77 R-4,70$ & 408.70 & -66 & $3.54 \mathrm{E}-03$ & -55.4 & 51 & $2.28 \mathrm{E}-04$ & -60.3 \\
\hline $78 \mathrm{R}-3,51$ & 412.79 & -76 & $2.74 \mathrm{E}-04$ & -59.7 & 78 & $1.14 \mathrm{E}-04$ & -60.1 \\
\hline $78 R-4,34$ & 414.12 & -24 & $2.05 \mathrm{E}-04$ & -59.8 & 72 & - & - \\
\hline $78 \mathrm{R}-4,65$ & 414.65 & -71 & $2.93 \mathrm{E}-04$ & -59.7 & 59 & $8.40 \mathrm{E}-05$ & -60.1 \\
\hline $79 \mathrm{R}-2,65$ & 416.65 & -85 & $8.04 \mathrm{E}-03$ & -49.4 & 43 & $1.29 \mathrm{E}-03$ & -61.6 \\
\hline
\end{tabular}


Table 2 (continued).

\begin{tabular}{|c|c|c|c|c|c|c|c|}
\hline $\begin{array}{l}\text { Core/section } \\
\text { interval }(\mathrm{cm})\end{array}$ & $\begin{array}{l}\text { Depth } \\
\text { (mbsf) }\end{array}$ & $\begin{array}{c}I_{n r m} \\
(\mathrm{kA} / \mathrm{m})\end{array}$ & $\begin{array}{c}J_{n r m} \\
(\mathrm{kA} / \mathrm{m})\end{array}$ & $I_{c}$ & $I_{s}$ & $\begin{array}{c}J_{s t} \\
(\mathrm{kA} / \mathrm{m})\end{array}$ & $I_{c s}$ \\
\hline $79 \mathrm{R}-4,12$ & 419.07 & -41 & $9.18 \mathrm{E}-04$ & -58.8 & 45 & $2.31 \mathrm{E}-05$ & -60.0 \\
\hline $79 \mathrm{R}-6,27$ & 422.05 & -60 & $2.79 \mathrm{E}-02$ & -5.2 & 68 & - & \\
\hline $79 \mathrm{R}-7,99$ & 424.49 & -60 & $1.03 \mathrm{E}-04$ & -59.9 & 54 & $6.00 \mathrm{E}-05$ & -60.1 \\
\hline $80 \mathrm{R}-1,131$ & 425.31 & -75 & $1.09 \mathrm{E}-03$ & -58.7 & 57 & $1.31 \mathrm{E}-04$ & -60.2 \\
\hline $80 \mathrm{R}-3,121$ & 428.18 & -54 & $3.27 \mathrm{E}-02$ & 2.4 & 36 & $9.70 \mathrm{E}-04$ & -61.2 \\
\hline $80 \mathrm{R}-7,23$ & 433.23 & -65 & $7.49 \mathrm{E}-03$ & -49.0 & 52 & $2.49 \mathrm{E}-04$ & -60.3 \\
\hline $81 \mathrm{R}-2,54$ & 435.54 & -68 & $2.30 \mathrm{E}-04$ & -59.7 & 71 & $2.22 \mathrm{E}-04$ & -60.2 \\
\hline $81 \mathrm{R}-3,37$ & 436.64 & -55 & $3.56 \mathrm{E}-04$ & -59.6 & 59 & - & - \\
\hline $81 \mathrm{R}-4,134$ & 439.11 & -30 & $3.32 \mathrm{E}-04$ & -59.6 & 70 & $2.56 \mathrm{E}-04$ & -60.3 \\
\hline $81 \mathrm{R}-7,64$ & 442.84 & -71 & $1.45 \mathrm{E}-04$ & -59.8 & - & - & - \\
\hline $82 \mathrm{R}-1,18$ & 443.18 & 62 & $6.06 \mathrm{E}-02$ & -89.4 & 80 & - & - \\
\hline $82 \mathrm{R}-2,13$ & 444.63 & -72 & $2.23 \mathrm{E}-04$ & -59.7 & 53 & $2.99 \mathrm{E}-04$ & -60.0 \\
\hline $82 R-5,45$ & 449.02 & -71 & $1.40 \mathrm{E}-02$ & -36.0 & - & - & - \\
\hline $82 R-6,11$ & 450.61 & -70 & $3.41 \mathrm{E}-02$ & 17.2 & 33 & $5.04 \mathrm{E}-03$ & -65.9 \\
\hline $83 R-2,105$ & 455.05 & 38 & $7.55 \mathrm{E}-04$ & -60.9 & 38 & $1.59 \mathrm{E}-03$ & -61.9 \\
\hline $83 R-4,95$ & 457.95 & -57 & $2.48 \mathrm{E}-04$ & -59.7 & 49 & $7.98 \mathrm{E}-05$ & -60.1 \\
\hline $83 R-7,104$ & 462.54 & -55 & $2.89 \mathrm{E}-04$ & -59.6 & 63 & 7.04E-05 & -60.1 \\
\hline $84 \mathrm{R}-2,67$ & 464.17 & -72 & $1.33 \mathrm{E}-03$ & -58.4 & 78 & $2.45 \mathrm{E}-04$ & -60.3 \\
\hline $84 \mathrm{R}-3,14$ & 465.14 & -46 & $5.96 \mathrm{E}-04$ & -59.2 & 72 & $9.43 \mathrm{E}-04$ & -61.1 \\
\hline $84 \mathrm{R}-4,23$ & 466.46 & -25 & $6.51 \mathrm{E}-04$ & -59.3 & 65 & - & - \\
\hline $84 \mathrm{R}-7,3$ & 470.14 & 44 & $1.25 \mathrm{E}-03$ & -61.5 & 73 & 1.49E-03 & -61.6 \\
\hline $85 \mathrm{R}-1,75$ & 472.25 & -60 & $5.96 \mathrm{E}-03$ & -51.7 & 71 & $6.08 \mathrm{E}-04$ & -60.7 \\
\hline $85 R-3,94$ & 475.39 & -60 & $3.71 \mathrm{E}-03$ & -55.1 & 60 & - & - \\
\hline $85 R-4,9$ & 476.09 & -68 & $1.48 \mathrm{E}-03$ & -58.1 & 80 & $9.95 \mathrm{E}-04$ & -61.1 \\
\hline $85 R-4,37$ & 476.11 & 49 & $3.08 \mathrm{E}-04$ & -60.4 & 80 & $5.18 \mathrm{E}-04$ & -60.5 \\
\hline $85 \mathrm{R}-7,17$ & 480.67 & -74 & $1.11 \mathrm{E}-03$ & -58.7 & 73 & $1.61 \mathrm{E}-04$ & -60.2 \\
\hline $86 \mathrm{R}-1,24$ & 481.24 & 33 & $9.92 \mathrm{E}-04$ & -61.2 & 47 & - & - \\
\hline $86 \mathrm{R}-3,79$ & 484.79 & -82 & $1.24 \mathrm{E}-02$ & -41.0 & 80 & - & - \\
\hline $86 \mathrm{R}-3,133$ & 485.33 & -36 & $7.84 \mathrm{E}-03$ & -49.7 & 66 & $2.62 \mathrm{E}-03$ & -63.0 \\
\hline $86 \mathrm{R}-6,143$ & 489.93 & -64 & $1.22 \mathrm{E}-02$ & -40.5 & 69 & $1.70 \mathrm{E}-04$ & -60.2 \\
\hline $87 \mathrm{R}-3,64$ & 493.98 & -12 & $4.85 \mathrm{E}-04$ & -59.5 & 69 & $9.20 \mathrm{E}-04$ & -61.1 \\
\hline $87 \mathrm{R}-3,115$ & 494.49 & -70 & 8.91E-04 & -58.9 & 76 & - & - \\
\hline $87 \mathrm{R}-5,11$ & 496.61 & -59 & $1.52 \mathrm{E}-02$ & -34.7 & 59 & $2.68 \mathrm{E}-04$ & -60.3 \\
\hline $87 \mathrm{R}-5,20$ & 496.70 & -79 & $1.65 \mathrm{E}-02$ & -28.6 & 70 & $7.95 \mathrm{E}-05$ & -60.1 \\
\hline $87 \mathrm{R}-6,28$ & 498.12 & -77 & $4.87 \mathrm{E}-03$ & -53.8 & 61 & - & - \\
\hline
\end{tabular}

$I_{n r m}, J_{n r m}$, and $I_{s}$ are the same as in Table $1 . I_{c}=$ the magnetic field inclination within a hole calculated using NRM data $\left(I_{n r m}\right.$ and $\left.J_{n r m}\right) . J_{s t}=$ the magnetization intensity of the demagnetization step when only the stable magnetic component is predominant. $I_{c s}$ is the magnetic field inclination calculated by using stable magnetization data $\left(I_{s}\right.$ and $\left.J_{s t}\right)$.

lie between 1 and 10 , as already mentioned for all of the samples in the previous section. This agrees with previous studies for gabbros from both the Kane Fracture Zone and the Troodos Ophiolite (Fox and Opdyke, 1973; Pariso and Johnson, 1989b) and shows that these in-situ magnetizations are dominated by a remanent magnetization.

MDF values determined from the studied samples having both positive NRM inclinations and thin section descriptions have been plotted as histograms in Figure 18. As the figure shows, most of the samples have MDF values higher than 15 $\mathrm{mT}$, indicating that the stability of natural remanence is good. Figure 18 also shows that relative high frequencies at lower ranges of MDFs can be seen in the medium- and high-grade samples, compared with the low-grade samples. This may indicate that samples having a higher metamorphism/alteration grade have lower coercive force.

The magnetic properties described above show that there is essentially no detectable difference among the three metamorphism/alteration grades, although perhaps a slightly less magnetic phase exists in the higher grades of metamorphism. MDF values indicate a good grouping at lower ranges in the higher grades. Because coercivity is strongly influenced by grain size and specific composition, defects, etc. in magnetic minerals, this may reveal that some changes in magnetic characteristic are a result of metamorphism/alteration, although these are undetectable from the changes of magnetic properties. To obtain magnetic properties undisturbed by drilling remanence, the samples having negative (normal) inclinations were not used for this discussion. This resulted in having both a smaller number of samples and in excluding most of the magnetite- and ilmenite-rich gabbros. More extensive data will be needed for further discussion.

\section{Oceanic Intrusive Rocks as the Origin of Lineated Magnetic Anomalies}

Although many types of rocks collected so far within fracture zones are thought to make up layer 3, gabbros predominate. The question of whether rocks from oceanic fracture zones or ophiolite complexes really represent normal layer 3 , or whether they are in some way anomalous remains open (Bonatti and Honnorez, 1976), but in this discussion, we accept the idea that gabbroic rocks make up layer 3. The magnetic studies for these rocks indicate that oceanic gabbros have high enough magnetic intensities to explain significant amounts of the magnetic anomaly as well as relatively stable magnetizations (Fox and Opdyke, 1973; Kent et al., 1978;, Dunlop and Prevot, 1982). Hayling and Harrison (1986) postulated that the arithmetic mean magnetizations of gabbros of various types are not very different, suggesting that the whole of layer 3 may be considered a possible source for seafloor spreading magnetic anomalies. As discussed above, this study also shows that gabbros from Hole $735 \mathrm{~B}$ have reasonably high and stable magnetizations. An important question not discussed in previous studies is the timing of the magnetization of oceanic gabbros, because metamorphism/alteration and serpentinization (which are 
INCLINATION

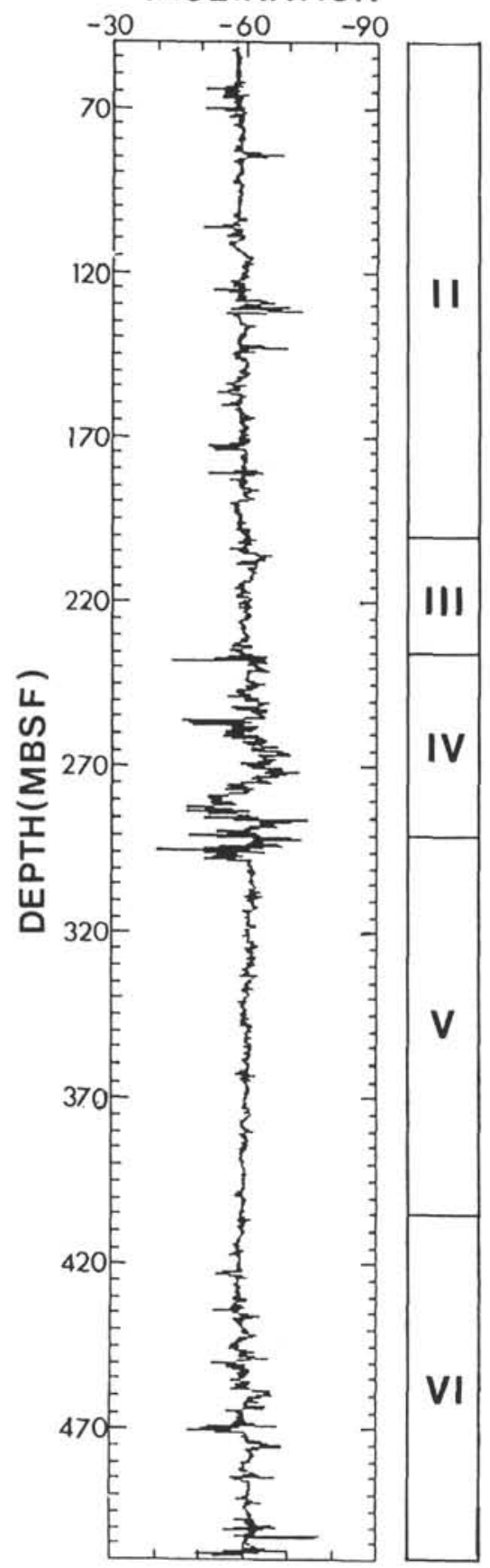

Figure 9. Magnetic inclination computed from the gyroinclinometer referenced magnetometer measurements within Hole 735B (Pariso et al., this volume; Robinson, Von Herzen, et al., 1989).

considered to have taken place sufficiently late after the formation of the oceanic crust at the ridge) can remagnetize the rocks, resulting in change of Vine-Matthews-Morley type initial magnetization acquired at the ridge. By studying vertically oriented samples from gabbros of various types that underwent various degrees of metamorphism/alteration, this investigation showed that the whole section drilled has a unique, stable polarity and that the mean stable inclination of $66^{\circ}$ is not so different from the value expected on the basis of the axial geomagnetic dipole field. This may indicate that metamorphism/alteration in Leg 118 oceanic gabbros prob-

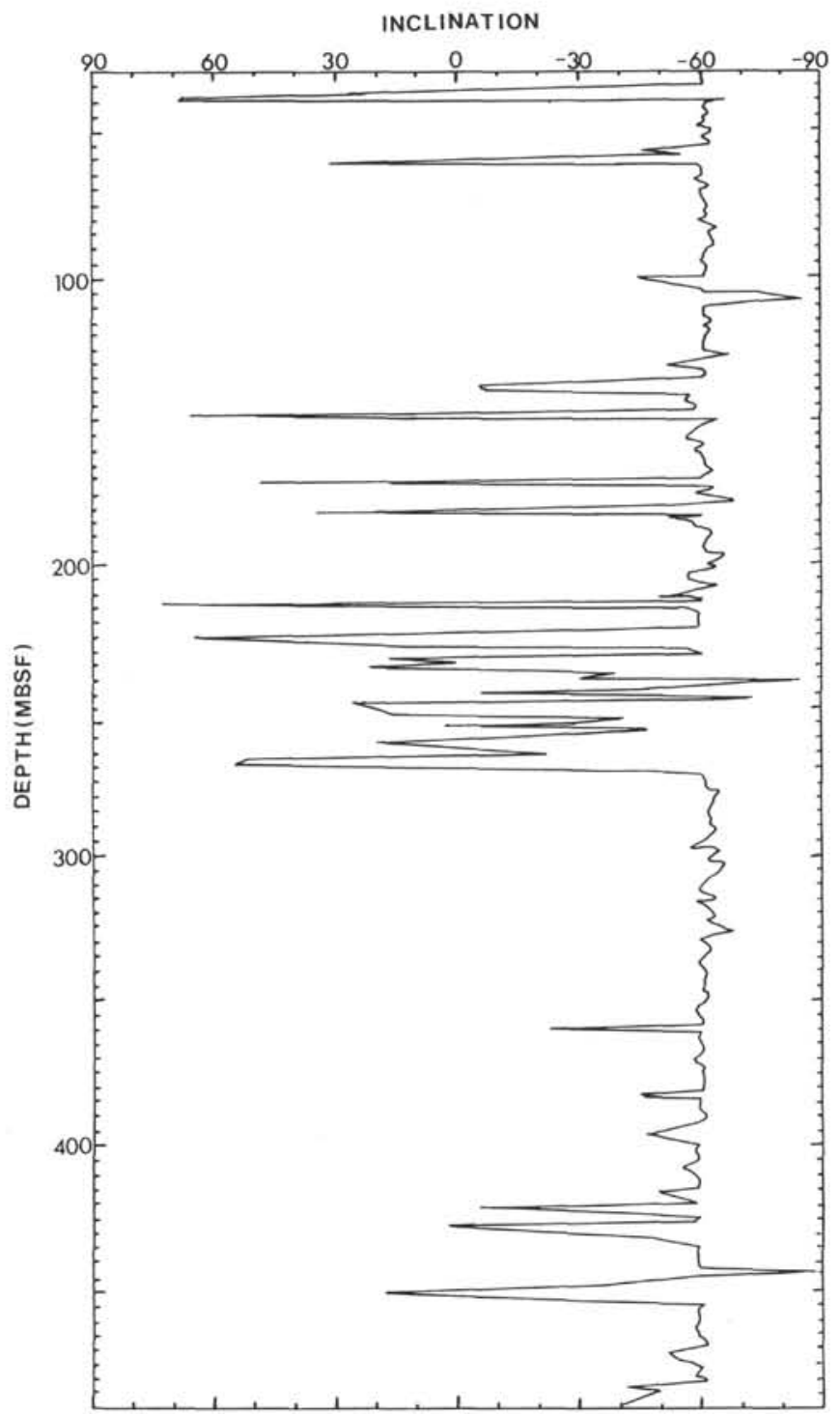

Figure 10. Magnetic inclinations in Hole 735B calculated from NRM data.

ably occurred within a relatively short time (perhaps during one of the reversed polarity chrons around anomaly 5A) after formation at the ridge. Miyashiro (1973) mentioned that ocean-floor metamorphism would take place mainly beneath the crest of mid-ocean ridges because the geothermal gradient would be relatively high there, which is consistent with this study. Contrasting with other DSDP/ODP studies of basalts having geomagnetic reversals in a vertical section, magnetizations acquired by Leg 118 oceanic gabbros are consistent with the Vine-Matthews-Morley type of hypothesis.

By assuming that layer 3 has been magnetized uniformly, we calculated the marine magnetic anomaly (Fig. 19). Because of the secondary component acquired during drilling, one cannot estimate in-situ magnetization by using NRM data (as conducted out in previous studies). As shown in the previous discussion, magnetic inclinations calculated using stable magnetization intensities $\left(J_{s t}\right)$ agree well with the magnetic logging measurement; thus, the average of $J_{s t}$ has been used as a magnetization of layer 3, although it indicates a lower value of the true mean than what it actually is. Figure 19 depicts three 
INCLINATION

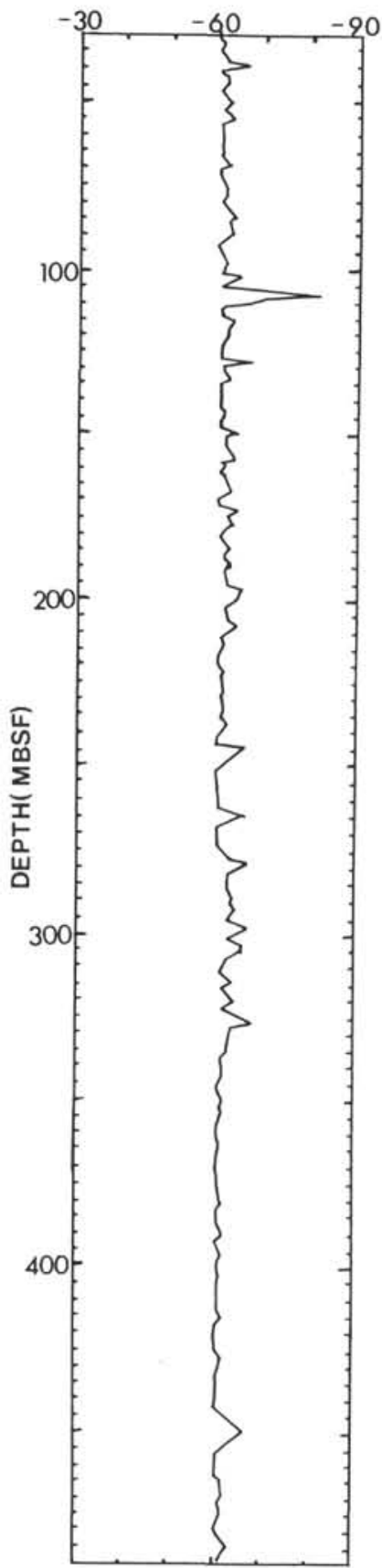

Figure 11. Magnetic inclinations in Hole 735B calculated from stable magnetization data.

marine magnetic anomaly profiles calculated on the basis of three models: a layer 2A, a whole layer 2, and a whole layer 3. All of the models presented in Figure 19 have had no contributions from other layers. In layer $2 \mathrm{~A}$ and layer 2 models, assumed remanence intensities are higher than the average means estimated in the previous studies, and thus magnetic amplitudes calculated for the two models in Figure 19 are slightly larger than expected. The uppermost profile was calculated by assuming a layer $2 \mathrm{~A}$ model $(0.5 \mathrm{~km}$ thickness and $8 \mathrm{~A} / \mathrm{m}$ magnetization). The middle profile is based upon a layer 2 model $(1.5 \mathrm{~km}$ and $3 \mathrm{~A} / \mathrm{m})$. The lowermost profile has been calculated from the layer 3 model proposed here $(4.5 \mathrm{~km}$

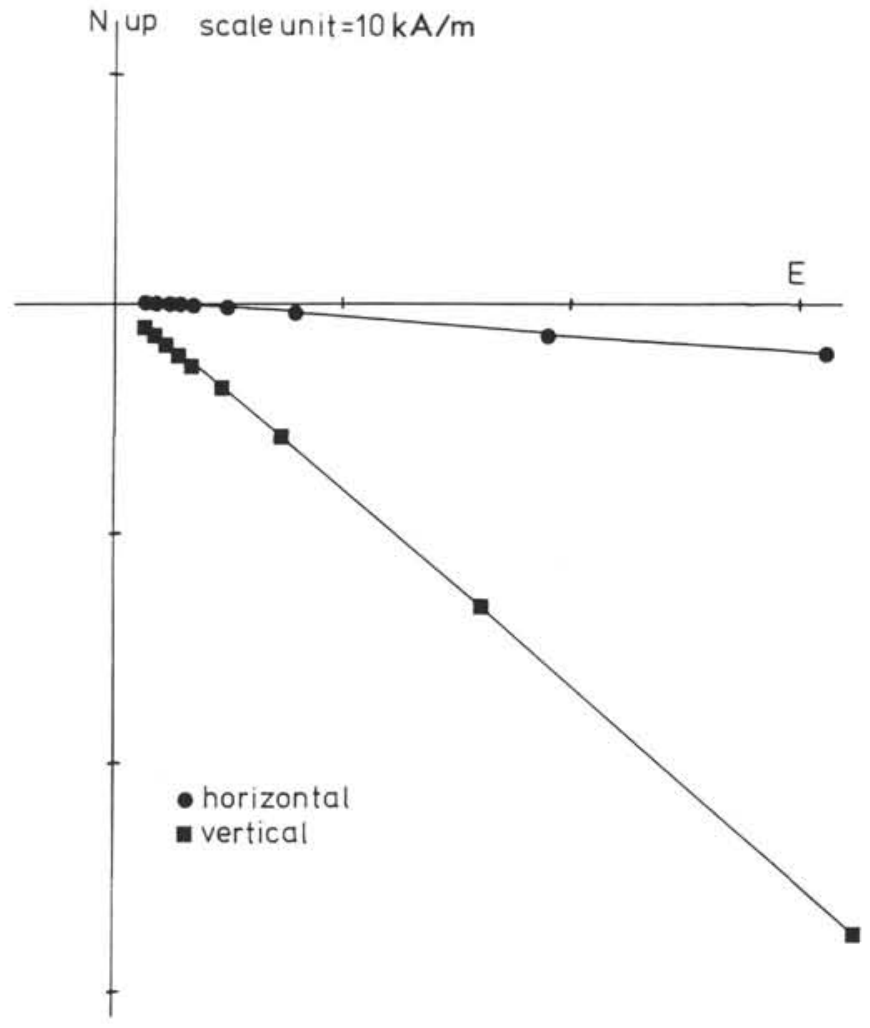

Figure 12. AF demagnetization data of thermoremanent magnetization applied to Sample 118-735B-47R-2, $111 \mathrm{~cm}$, in a magnetic field of $0.5 \mathrm{Oe}$, with heating up to $600^{\circ} \mathrm{C}$. Demagnetization steps are 5,10 , $15,20,25,30,40$, and $50 \mathrm{mT}$. The Zijderveld plot clearly shows good stability of applied TRM against alternating field.

and $1.6 \mathrm{~A} / \mathrm{m})$. As Figure 19 shows, the calculated magnetic anomalies agree with one another, meaning that layer 3 has a magnetization capable of producing most of the marine magnetic anomaly.

\section{CONCLUSIONS}

Knowledge of magnetization intensity in rocks dredged or drilled from the ocean floor and in samples from ophiolite complexes indicates that oceanic extrusive basalt are unlikely to be the sole source for marine magnetic anomalies. Extrusive lavas are unlikely to have sufficient magnetization to explain the amplitude of marine magnetic anomalies because of the effects of alteration and the interlayering of different magnetic polarities in a vertical section that integrates to effectively lower magnetic intensities. Concerning the layer composed of diabase (sheeted dike complex, layer 2B), its contribution to the anomaly is much less than that of the oceanic extrusive basalt because of its low magnetic intensity caused by both the larger grain size of magnetic minerals included in diabase and the metamorphism of these rocks. Several scientists, however, reported that unaltered diabase had magnetization intensity values high enough to produce the anomaly amplitude. A detailed study of hydrothermal alteration of titanomagnetite indicated that this process altered original titanomagnetite and caused recrystalization of Ti-poor magnetite, resulting in a change of primary magnetic direction and loss of sufficient amount of initial thermoremanent magnetization (Fujimoto and Kikawa, 1989). Hydrothermal alteration is a key process in the metamorphism of oceanic rocks. If the percentage of oceanic rocks that underwent metamor- 
Table 3. Metamorphism and alteration grades of samples from Hole 735B.

\begin{tabular}{|c|c|c|}
\hline $\begin{array}{l}\text { Core, section, } \\
\text { interval }(\mathrm{cm})\end{array}$ & $\begin{array}{l}\text { Total percentage of secondary } \\
\text { hydrous minerals }(\%)\end{array}$ & $\begin{array}{c}\text { Metamorphism/alteration } \\
\text { grade }^{\mathrm{a}}\end{array}$ \\
\hline 118-735B- & & \\
\hline 1D-1, 19 & 15 & Medium \\
\hline $1 D-1,141$ & 20 & Medium \\
\hline $2 \mathrm{D}-1,139$ & 22 & Medium \\
\hline $2 \mathrm{D}-2,99$ & 19 & Medium \\
\hline $3 \mathrm{D}-1,42$ & 47 & High \\
\hline $3 \mathrm{D}-1,58$ & 39 & High \\
\hline $3 \mathrm{D}-1,60$ & 39 & High \\
\hline $4 \mathrm{D}-2,7$ & 28 & High \\
\hline $6 \mathrm{D}-1,111$ & 42 & High \\
\hline $7 \mathrm{D}-1,74$ & 37.2 & High \\
\hline $8 \mathrm{D}-1,45$ & 24 & Medium \\
\hline $9 D-1,120$ & 17 & Medium \\
\hline $10 \mathrm{D}-1,24$ & 19 & Medium \\
\hline IID-1, 3 & 51 & High \\
\hline $11 D-1,6$ & 51 & High \\
\hline $12 \mathrm{R}-1,32$ & 6 & Low \\
\hline $12 \mathrm{R}-2,36$ & 56 & High \\
\hline $12 \mathrm{R}-3,8$ & 13 & Medium \\
\hline $12 \mathrm{R}-3,83$ & 4 & Low \\
\hline $13 \mathrm{R}-1,102$ & 23 & Medium \\
\hline $13 R-2,55$ & 9.8 & Low \\
\hline $14 \mathrm{R}-1,35$ & 22 & Medium \\
\hline $14 \mathrm{R}-2,22$ & 17 & Medium \\
\hline $14 \mathrm{R}-4,22$ & 3 & Low \\
\hline ISR-2, 128 & 25 & High \\
\hline $16 R-4,77$ & 40 & High \\
\hline $16 \mathrm{R}-5,24$ & 18 & Medium \\
\hline $18 \mathrm{R}-3,29$ & 53 & High \\
\hline $19 \mathrm{R}-5,126$ & 55 & High \\
\hline $20 \mathrm{R}-2,54$ & 1.8 & Low \\
\hline $21 \mathrm{R}-2,49$ & 7 & Low \\
\hline $22 \mathrm{R}-2,41$ & 40 & High \\
\hline $22 \mathrm{R}-3,118$ & 39.4 & High \\
\hline $23 R-2,34$ & 9.1 & Low \\
\hline $23 \mathrm{R}-4,120$ & 18.3 & Medium \\
\hline $24 \mathrm{R}-2,95$ & 34 & High \\
\hline $24 \mathrm{R}-3,50$ & 40 & High \\
\hline $24 \mathrm{R}-4,26$ & 27.8 & High \\
\hline $25 \mathrm{R}-2,5$ & 45 & High \\
\hline $25 \mathrm{R}-3,137$ & 14 & Medium \\
\hline $26 \mathrm{R}-1,62$ & 41 & High \\
\hline $27 \mathrm{R}-1,84$ & 11 & Medium \\
\hline $27 \mathrm{R}-3,32$ & 21 & Medium \\
\hline 28R-2, 12 & 10 & Medium \\
\hline $28 \mathrm{R}-2,114$ & 24 & Medium \\
\hline $29 \mathrm{R}-2,46$ & 14 & Medium \\
\hline $29 \mathrm{R}-4,19$ & 32 & High \\
\hline $30 \mathrm{R}-3,137$ & 18 & Medium \\
\hline $30 \mathrm{R}-4,14$ & 29 & High \\
\hline $30 \mathrm{R}-5,91$ & 10 & Medium \\
\hline $31 \mathrm{R}-2,120$ & 27 & High \\
\hline $31 \mathrm{R}-4,118$ & 31 & High \\
\hline $32 \mathrm{R}-1,64$ & 19 & Medium \\
\hline $32 \mathrm{R}-3,14$ & 7 & Low \\
\hline $33 R-4,27$ & 14 & Medium \\
\hline $33 \mathrm{R}-4,129$ & 57 & High \\
\hline $34 \mathrm{R}-1,103$ & 8.5 & Low \\
\hline $34 \mathrm{R}-4,30$ & 28 & High \\
\hline $35 \mathrm{R}-1,29$ & 16.2 & Medium \\
\hline $35 \mathrm{R}-5,131$ & 12 & Medium \\
\hline $36 \mathrm{R}-1,14$ & 40 & High \\
\hline $36 \mathrm{R}-2,11$ & 9 & Low \\
\hline $36 \mathrm{R}-3,36$ & 10 & Medium \\
\hline $37 \mathrm{R}-1,11$ & 21 & Medium \\
\hline $37 \mathrm{R}-3,80$ & 11 & Medium \\
\hline $38 \mathrm{R}-2,15$ & 30 & High \\
\hline $39 \mathrm{R}-1,145$ & 6 & Low \\
\hline $39 \mathrm{R}-3,21$ & 26 & High \\
\hline $40 \mathrm{R}-2,62$ & 16 & Medium \\
\hline $40 \mathrm{R}-5,13$ & 2 & Low \\
\hline $41 \mathrm{R}-4,68$ & 3 & Low \\
\hline $42 R-2,119$ & 3.9 & Low \\
\hline $42 \mathrm{R}-4,62$ & 3.5 & Low \\
\hline $43 \mathrm{R}-1,126$ & 35 & High \\
\hline $43 R-4,64$ & 13 & Medium \\
\hline $44 \mathrm{R}-2,6$ & 11 & Medium \\
\hline $44 \mathrm{R}-2,131$ & 22 & Medium \\
\hline
\end{tabular}

Table 3 (continued).

\begin{tabular}{|c|c|c|}
\hline $\begin{array}{l}\text { Core, section, } \\
\text { interval }(\mathrm{cm})\end{array}$ & $\begin{array}{c}\text { Total percentage of secondary } \\
\text { hydrous minerals }(\%)\end{array}$ & $\begin{array}{c}\text { Metamorphism/alteration } \\
\text { grade }^{\mathrm{a}}\end{array}$ \\
\hline $45 \mathrm{R}-2,15$ & - & Low \\
\hline $46 \mathrm{R}-2,128$ & 13 & Medium \\
\hline $47 R-3,50$ & 2 & Low \\
\hline $48 \mathrm{R}-4,82$ & 8 & Low \\
\hline $49 \mathrm{R}-1,36$ & 7.2 & Low \\
\hline $49 \mathrm{R}-2,89$ & 13 & Medium \\
\hline $50 \mathrm{R}-4,87$ & 5.5 & Low \\
\hline $51 \mathrm{R}-1,102$ & 10 & Medium \\
\hline $54 \mathrm{R}-3,125$ & 12 & Medium \\
\hline $54 \mathrm{R}-5,117$ & 9 & Low \\
\hline $55 \mathrm{R}-3,130$ & 5 & Low \\
\hline $56 \mathrm{R}-2,11$ & 3 & Low \\
\hline 56R-2, 144 & 30 & High \\
\hline 57R-2, 135 & 27 & High \\
\hline $58 \mathrm{R}-2,33$ & 6 & Low \\
\hline $59 \mathrm{R}-3,70$ & 2 & Low \\
\hline $60 \mathrm{R}-1,18$ & - & Low \\
\hline $61 \mathrm{R}-1,81$ & 2 & Low \\
\hline $62 \mathrm{R}-3,104$ & 16 & Medium \\
\hline $63 \mathrm{R}-3,80$ & 9 & Low \\
\hline $63 R-6,28$ & 38 & High \\
\hline $64 \mathrm{R}-2,54$ & 6 & Low \\
\hline $65 \mathrm{R}-3,61$ & 1.1 & Low \\
\hline $66 \mathrm{R}-2,86$ & 30 & High \\
\hline $66 \mathrm{R}-3,60$ & 38 & High \\
\hline $66 \mathrm{R}-3,134$ & 11 & Medium \\
\hline $69 \mathrm{R}-4,138$ & 12 & Medium \\
\hline $70 \mathrm{R}-1,105$ & 20 & Medium \\
\hline $71 \mathrm{R}-2,82$ & - & Low \\
\hline $71 \mathrm{R}-3,98$ & 53 & High \\
\hline $72 R-6,106$ & - & Low \\
\hline $73 \mathrm{R}-3,73$ & 3 & Low \\
\hline $74 \mathrm{R}-2,38$ & 3 & Low \\
\hline $74 \mathrm{R}-6,41$ & 1.7 & Low \\
\hline $75 R-6,75$ & 2.5 & Low \\
\hline $76 \mathrm{R}-3,50$ & 16 & Medium \\
\hline $78 \mathrm{R}-4,65$ & 1 & Low \\
\hline $79 \mathrm{R}-7,99$ & - & Low \\
\hline $80 \mathrm{R}-7,23$ & 9 & Low \\
\hline $81 R-2,54$ & 2 & Low \\
\hline $81 \mathrm{R}-7,64$ & 4 & Low \\
\hline $82 \mathrm{R}-2,13$ & 1 & Low \\
\hline $82 \mathrm{R}-6,11$ & 7 & Low \\
\hline $83 R-4,95$ & 2 & Low \\
\hline $83 \mathrm{R}-7,104$ & 30 & High \\
\hline $84 \mathrm{R}-3,14$ & 10 & Medium \\
\hline $85 R-4,9$ & 15 & Medium \\
\hline $85 \mathrm{R}-7,17$ & 12.6 & Medium \\
\hline $86 \mathrm{R}-6,143$ & 7 & Low \\
\hline $87 R-5,20$ & 30 & High \\
\hline
\end{tabular}

${ }^{\text {a }}$ Metamorphism/alteration grades have been classified into three categories on the basis of the total percentage of secondary hydrous minerals (clay, chlorite. hornblende, amphibole, talc, epidote, tremolite, and actinolite): low grade includes $0 \%$ to $10 \%$ hydrous materials, and medium and high grades contain $10 \%$ to $25 \%$ and more than $25 \%$, respectively.

phism is much less than that observed, layer 2 as a whole (pillow and massive basalts and sheeted dikes) may contribute significantly to seafloor spreading magnetic anomalies. In spite of their stable and strong magnetization, some doubts exist whether oceanic gabbros are responsible for magnetic anomalies, because these results are based upon only a few studies and the time of the magnetization from metamorphism is uncertain. By recovering $500.7 \mathrm{~m}$ of continuous vertical oceanic gabbro section, Leg 118 first allowed us to measure magnetic properties of 264 minicores (one measurement per $1.9 \mathrm{~m}$ ) obtained from these rocks including their magnetic direction. Gabbros of various types that underwent various degrees of metamorphism showed that essentially there is no difference among the magnetic properties. The whole section drilled has stable magnetic inclinations with unique polarity, indicating that metamorphism in oceanic gabbros probably 


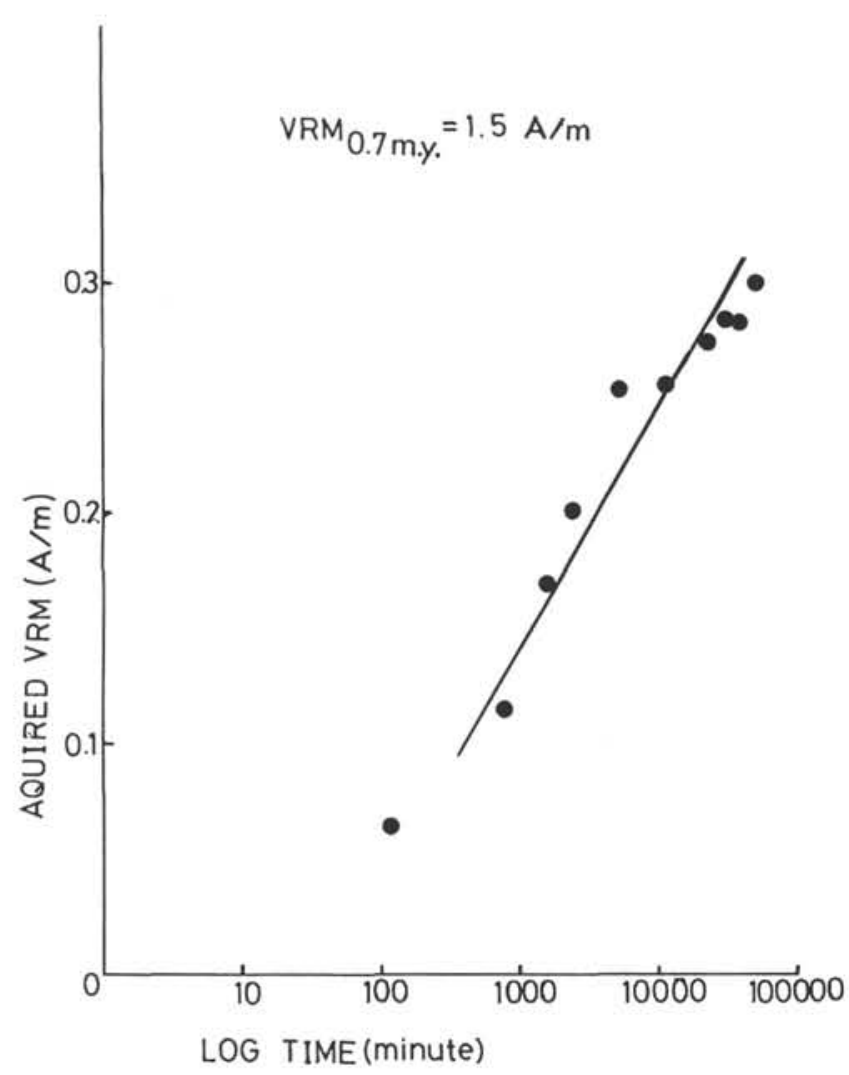

Figure 13. Acquisition of viscous remanence data during six-week storage test in a magnetic field of 0.5 Oe (Sample 118-735B-80R-3, 124 $\mathrm{cm})$. Experiments were performed after AF demagnetization. VRM acquisition during the Brunhes epoch $(0.7 \mathrm{~m} . \mathrm{y}$.) calculated on the basis of the least-square approximation of data plotted in the figure is $1.5 \mathrm{~A} / \mathrm{m}$.

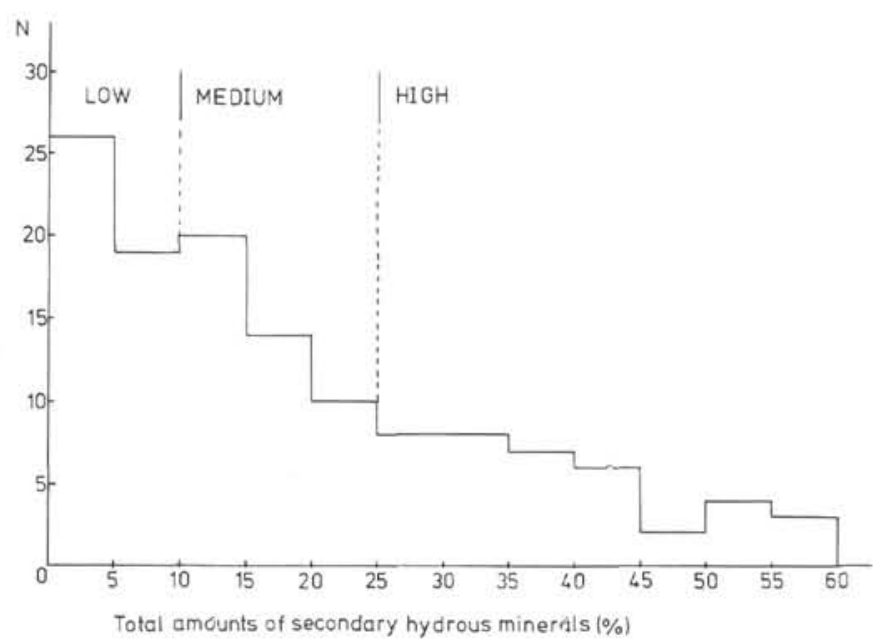

Figure 14. Histogram of total amounts of secondary hydrous minerals (\%) from which the metamorphism/alteration grade is determined. Samples containing less than $10 \%, 10 \%-25 \%$, and more than $25 \%$ are defined as low, medium, and high grades, respectively.

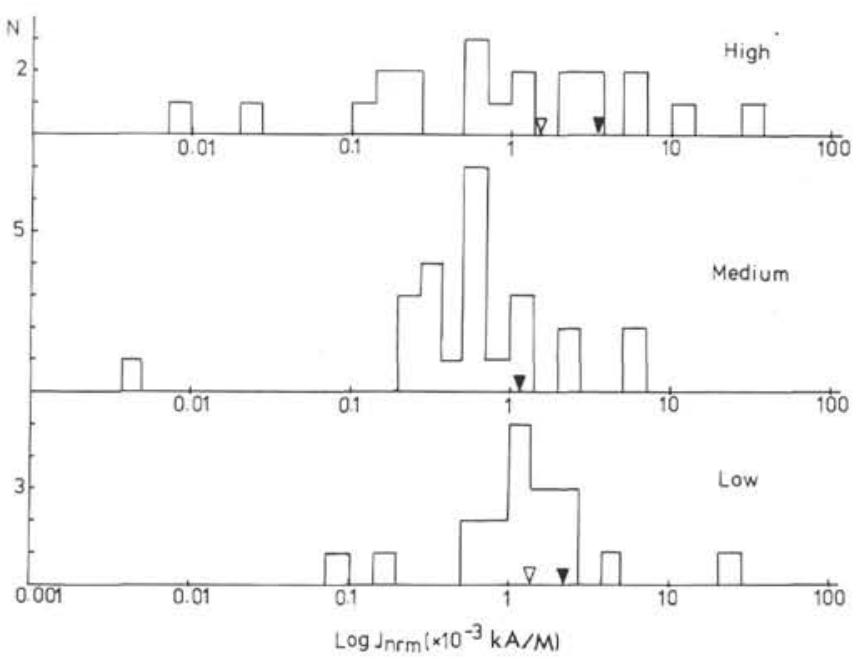

Figure 15. Histograms of natural remanent intensity. The upper, middle, and lower histograms indicate distributions for samples belonging to high, medium, and low metamorphism/alteration grades, respectively. Closed triangles = arithmetic means calculated for each grade; open triangles $=$ averages after excluding the highest samples of each grades .

occurs within a relatively short time after their formation at the ridge. Therefore, we conclude that oceanic gabbros acquire the so-called Vine-Matthews-Morley type of initial magnetization at the ridge. Moreover, their remanent magnetic intensities are strong enough to contribute to the marine magnetic anomaly. Even the average value of stable magnetizations $\left(J_{s t}\right)$ of Leg 118 gabbros, which we consider the

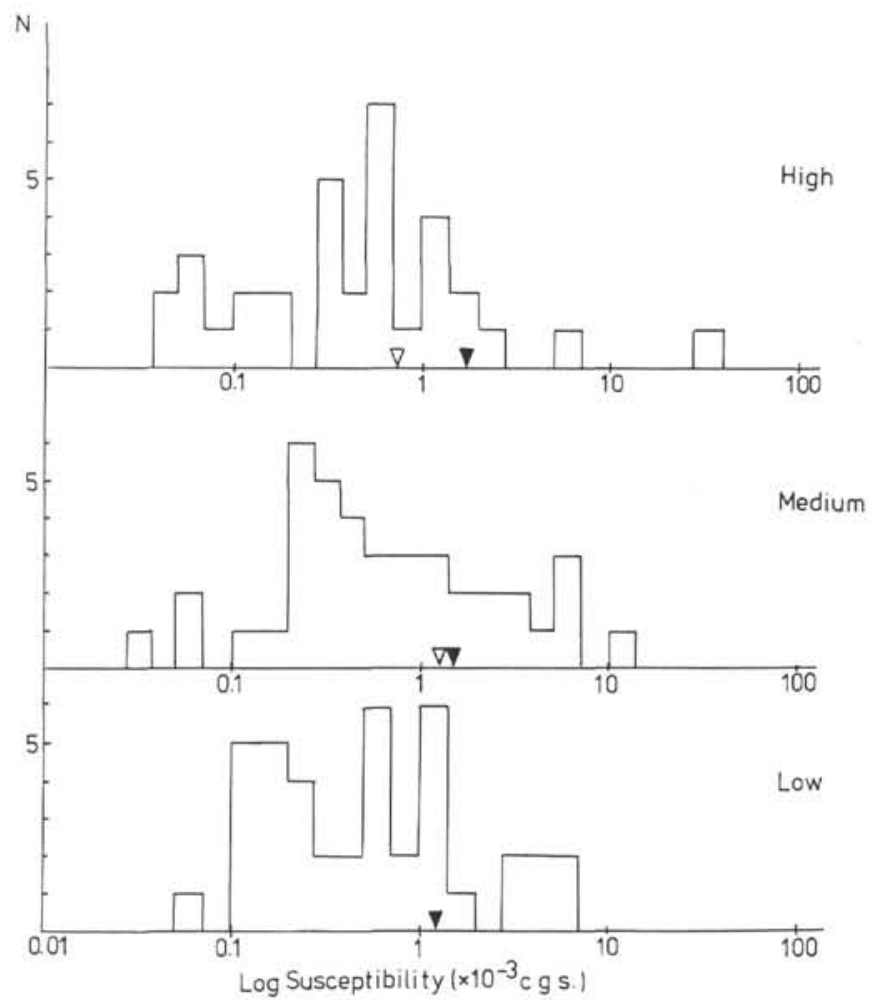

Figure 16. Histograms of initial magnetic susceptibility. Figure format same as in Figure 15. 


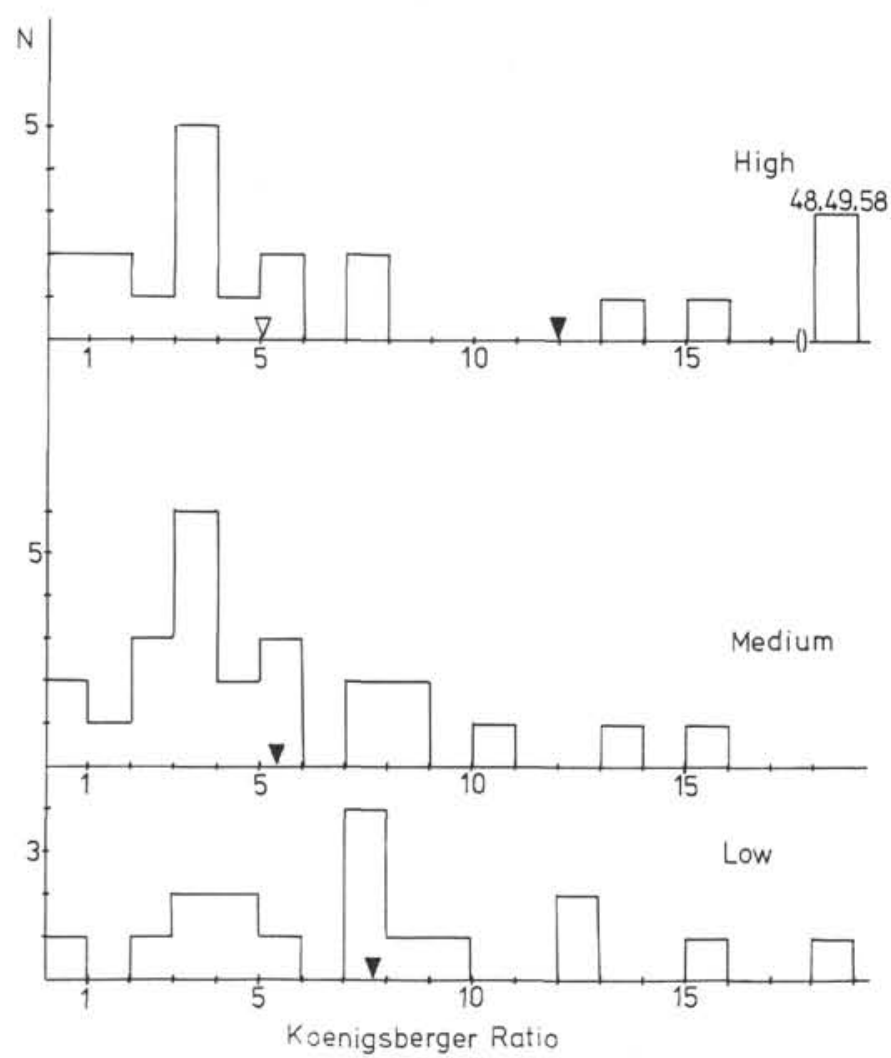

Figure 17. Histograms of Koenigsberger ratio. Figure format same as in Figure 15.

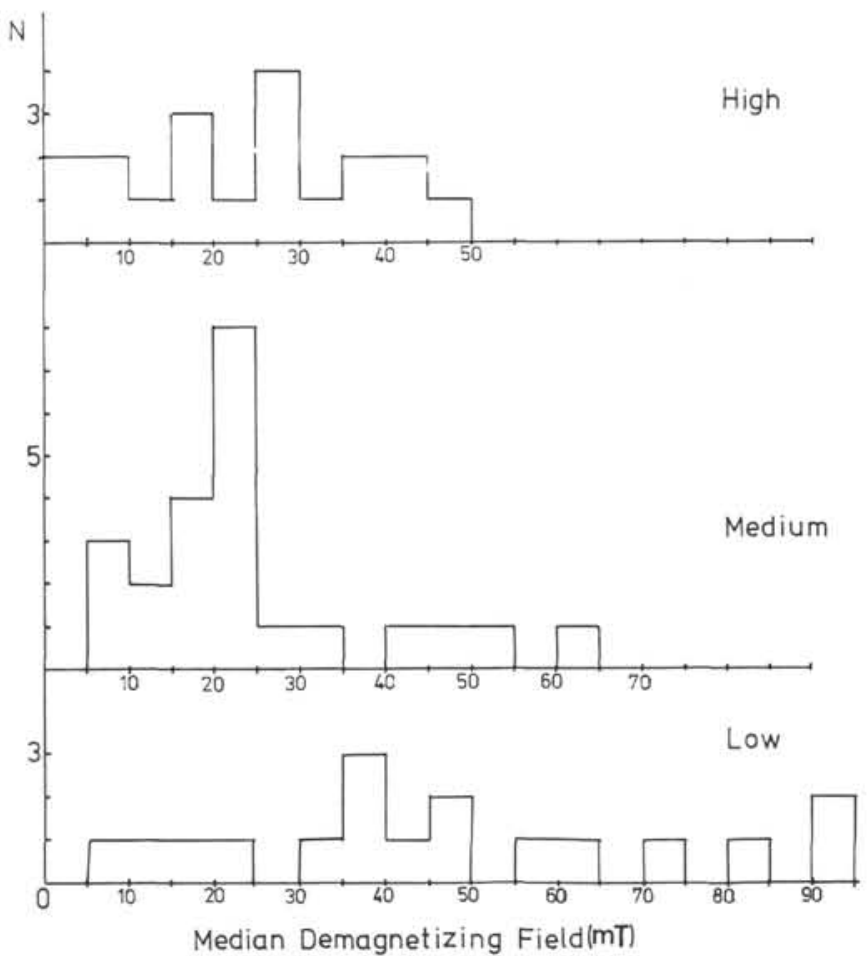

Figure 18. Histograms of median demagnetizing field. Figure format same as in Figure 15. minimum estimation, is $1.6 \mathrm{~A} / \mathrm{m}$. By assuming that layer 3 has been magnetized uniformly with this magnetization value, most of the marine magnetic anomaly amplitudes can be reproduced. Finally, we conclude that significant contribution to seafloor spreading anomalies may come from the oceanic gabbroic layer (layer 3 ) if the magnetic properties obtained during ODP Leg 118 are common features.

\section{ACKNOWLEDGMENTS}

We thank Seiya Uyeda and Yozo Hamano especially for providing valuable discussions and comments. We are also grateful to Tokihiko Matsuda for advice and help with the experiments. E. Kikawa wishes to expresses his appreciation to Asahiko Taira for giving him the opportunity to participate in Leg 118 . We thank the Leg 118 scientific party and technical staff for their assistance at sea. We are obliged to Kazuhito Ozawa, Tetsuro Urabe, and James H. Natland for their advice in classifying the metamorphism/alteration grades of recovered gabbros. Rie Morijiri, Masataka Kinoshita, and Makoto Uyeshima kindly helped in calculating the magnetic anomaly. This work was supported in part by NSF Grant No. OCE- 8609260 and by the JOI/USSAC fund for post-cruise science support. J. Pariso was supported by a JOI/USSAC Fellowship for much of the post-cruise period. Reviews by Paul J. Fox, Christopher G. A. Harrison, two anonymous reviewers, and the ODP editor helped to improve the manuscript.

\section{REFERENCES}

Atwater, T., and Mudie, J. D., 1973. Detailed near-bottom geophysical study of the Gorda Rise. J. Geophys. Res., 78:8665-8686.

Audunsson, H., and Levi, S., 1989. Drill-induced remanent magnetization in basalt drill cores. Geophys. J., 98:613-622.

Banerjee, S. K., 1980. Magnetization of the oceanic crust: Evidence from ophiolite complexes, J. Geophys. Res., 85:3557-3566. 1984. The magnetic layer of the ocean crust-How thick is it? Tectonophysics, 105:15-27.

Bonatti, E., and Honnorez, J., 1976. Sections of the Earth's crust in the equatorial Atlantic. J. Geophys. Res., 81:4104-4116.

Bosum, W., and Eberle, H., 1983. Bau und Erprobung eines 3-D Bohrlochmagnetometers. Geol. Jahrb., Hannover, E28:191-217.

Dunlop, D. J., and Prevot, M., 1982. Magnetic properties and opaque mineralogy of drilled submarine intrusive rocks. Geophys. J. Royal Astron. Soc., 69:763-802.

Fox, P. J., and Opdyke, N. D. 1973. Geology of the oceanic crust: Magnetic properties of oceanic rocks. J. Geophys. Res., 78:51395154.

Fujimoto, K., and Kikawa, E. 1989. Alteration of titanomagnetites and its related magnetic properties in the Noya Geothermal area, central Kyushu, Japan. J. Geomag. Geoelectr., 41:39- 64.

Harrison, C.G.A., 1981. Magnetism of the oceanic crust. In Emiliani, C. (Ed.), The Sea, Vol. 7: New York (Wiley), 219-239.

1987. Marine magnetic anomalies-the origin of the stripes. Annu. Rev. Earth Planet. Sci., 15:505-543.

Hayling, K. L., and Harrison, C.G.A., 1986. Magnetization modeling in the North and equatorial Atlantic Ocean using MAGSAT data. J. Geophys. Res., 91:12423-12443.

International Geomagnetic Reference Field, 1980: Scotland (Inst. of Geol. Sci.).

Kent, D. V., Honnorez, B. M., Opdyke, N. D., and Fox, P. J., 1978. Magnetic properties of dredged oceanic gabbros and source of marine magnetic anomalies. Geophys. J. Royal Astron. Soc., 55:513-537.

Koyama, M., 1986. Tectonic history of the Izu Peninsula and adjacent areas based on paleomagnetism and stratigraphy [Ph.D. dissert]. Geol. Inst., Univ. of Tokyo.

McFadden, P. L., and Reid, A. B., 1982. Analysis of paleomagnetic inclination data. Geophys. J. Royal Astron. Soc., 69:307-319.

Merrill, R. T., and McElhinny, M. W., 1983. The Earth's Magnetic Field: London (Academic Press), 20-23. 
Miyashiro, A., 1973. Metamorphism and Metamorphic Belts: London (George Allen \& Unwin Ltd), 26-27.

Pariso, J. E., and Johnson, H. P., 1989a. Magnetic properties and oxide petrography of the sheeted dike complex in Hole 504B. In Becker, K., Sakai, H., et al., 1990. Proc. ODP, Sci. Results, 111: College Station, TX (Ocean Drilling Program), 159-167.

1989b. Magnetic properties of an analog of the lower oceanic crust: magnetic logging and paleomagnetic measurements from drillhole CY-4 in the Troodos Ophiolite. Init. Repts. Cypress Crustal Study Project, Hole CY-4, 279-293.

Robinson, P. T., Von Herzen, R., et al., 1989. Proc. ODP, Init. Repts., 118: College Station, TX (Ocean Drilling Program).

Shipboard Scientific Party, 1989. Site 735. In Robinson, P. T., Von Herzen, R., et al., Proc. ODP, Init. Repts., 118: College Station, TX (Ocean Drilling Program), 670-816.

Smith, G. M., and Banerjee, S. K., 1986. Magnetic structure of the upper kilometers of the marine crust at Deep Sea Drilling Project
Hole 504B, Eastern Pacific Ocean. J. Geophys. Res., 91:1033710354.

Talwani, M., Windisch, C. C., and Langseth, M. G., Jr., 1971. Reykjanes Ridge crest: a detailed geophysical study. J. Geophys. Res., 76:473-517.

Tivey, M., and Johnson, P., 1984. The characterization of viscous remanent magnetization in large and small magnetite particles. $J$. Geophys. Res., 89:543-552.

Zijderveld, J.D.A., 1967. AC demagnetization of rocks: analysis and results. In Collinson, D. W., Creer, K. M., and Runcorn, S. K. (Eds.), Methods in Paleomagnetism: Amsterdam (Elsevier), 254286.

Date of initial receipt: 27 October 1989

Date of acceptance: 19 June 1990

Ms 118B-148 


\section{CALCULATED MARINE MAGNETIC ANOMALIES}

\section{Amplitude (nT)}
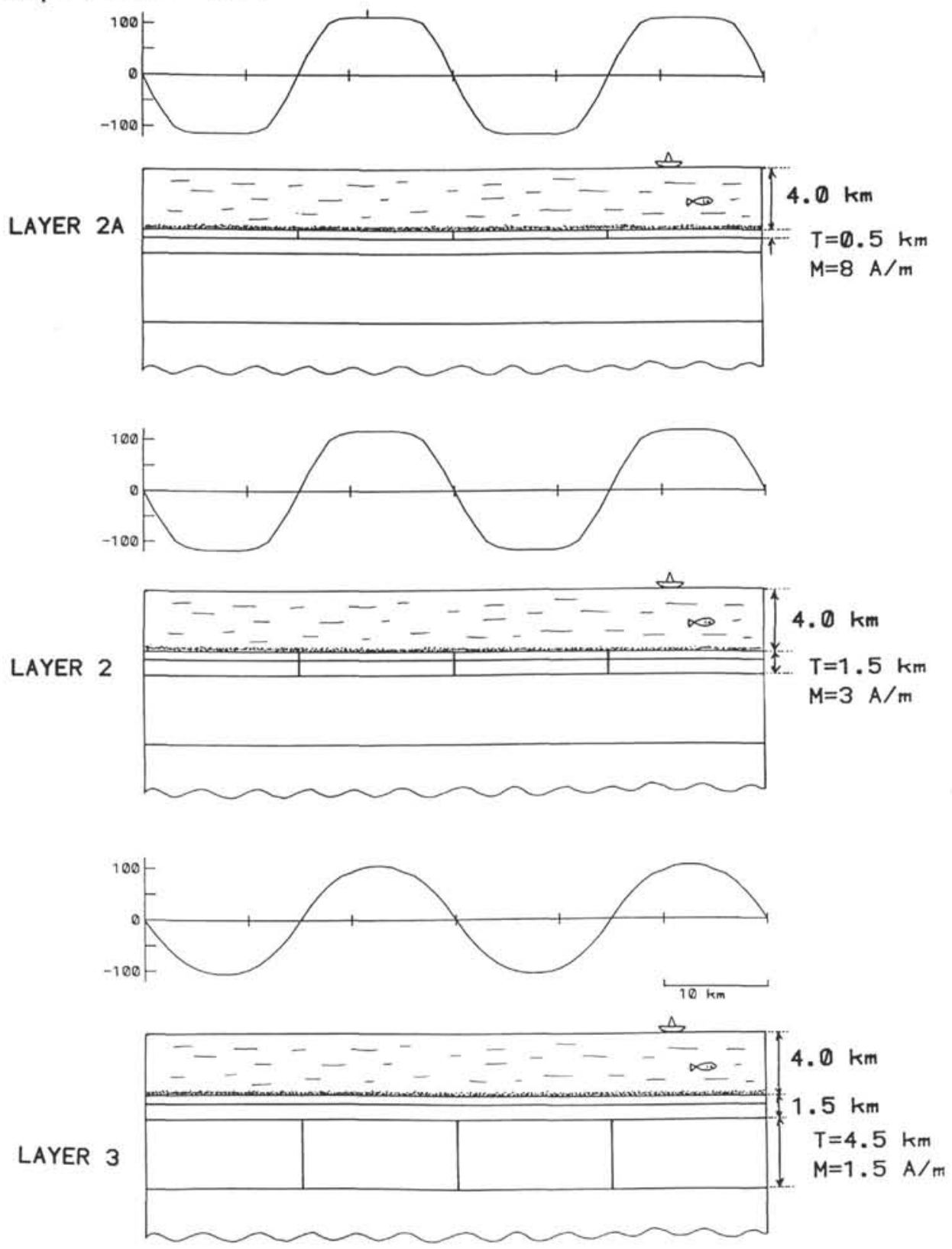

Figure 19. Marine magnetic anomalies calculated from: layer $2 \mathrm{~A}$ model (upper), layer 2 model (middle), layer 3 model (lower). The track line is set at $35^{\circ} \mathrm{N}$ in the east-west direction. Water depth is $4.0 \mathrm{~km}$, and no sediment layer has been considered. Assumed parameters are (1) thickness of magnetic layer $(T)$ and (2) intensity of magnetization $(M)$. 